\title{
Unbiasing a Stochastic Endmember Interpolator Using ENVI Object-Based Classifiers, a Farquhar's Single Voxel Leaf Photosynthetic Response Explanatory Model and Boolean Time Series Statistics for Forecasting Shade-Canopied Simulium damnosum s.l. Larval Habitats in Burkina Faso
}

Benjamin Jacob ${ }^{1 *}$, Robert J Novak ${ }^{1}$ Laurent Toe ${ }^{2}$, Moussa S Sanfo², Semiha Caliskan¹, Alain Pare ${ }^{3}$, Mounkaila Noma ${ }^{4}$, Laurent Yameogo ${ }^{4}$ and Thomas Unnasch ${ }^{1}$

${ }^{1}$ Global Infectious Disease Research Program, Department of Public Health, College of Public Health, University of South Florida, Tampa, Florida, USA Onchocerciasis Control Programme (OCP), Ouagadougou, Burkina Faso, West Africa

${ }^{3}$ Multi Disease Surveillance Centre, Ouagadougou, Burkina Faso, West Africa

${ }^{4}$ African Program for Onchocerciasis Control (APOC), Epidemiology and Vector Elimination, Ouagadougou, Burkina Faso, West Africa

\begin{abstract}
Endmember spectra recovered from sub-meter resolution data [e.g., QuickBird visible and near infra-red (NIR) $0.61 \mathrm{~m}$ waveband ratio] of an arthropod-related infectious disease aquatic larval habitat can act as a dependent variable within a least squares estimation algorithm. Consequently, seasonal endemic transmission-oriented risk variables can be accurately interpolated. Spectral mixing, however, is a problem inherent to multi-dimensional canopy-oriented arthropod-related infectious disease larval habitat feature attributes resulting in few image sub-pixel spectra representing "pure" targets. This can lead to a biased endmember target signature due to spectrally unquantitated mixed sub-pixel (i.e., mixel) radiance originating from different canopy-oriented larval habitat object types. An erroneous endmember sub-mixel larval habitat signature renders inconsistent residual forecasts in a stochastic/ deterministic interpolator. In these analyses, we spectrally extracted and decomposed multiple canopied endmembers surface-oriented sub-meter resolution pixel reflectance values derived from a georeferenced QuickBird imaged canopied larval habitat of Similium damnosum s.l., a black fly vector of onchocerciasis in an epidemiological riverine study site in Burkina Faso. We employed ENVI object-based classifiers, a 3-Dimensional radiative transfer equation and the Li-Strahler geometric-optical model to perform the decomposition. Thereafter, the georeferenced larval habitat and the canopy radiance values (e.g., Precambrian rock) were spectrally isolated and weighed using a robust Successive Progression Algorithm (SPA) within a Boolean domain. The decomposed endmembers rendered a robust spectral signature in ArcGIS which subsequently kriged to identify unknown, unsampled productive S. damnosum s.I. larval habitats along a Burkina Faso river system using a blind study format. The validation model revealed a $100 \%$ correlation among the predicted georeferenced productive black fly habitat sites based on the seasonal-sampled larval density count values.
\end{abstract}

Keywords: Endmembers; Mixels; Successive progression algorithm; Quick bird; Similium damnsoum s.l.; Burkina Faso

\section{Introduction}

Onchocerciasis, or River Blindness, is a neglected tropical disease caused by the parasitic worm Onchocerca volvulus. Estimates project 37 million individuals worldwide as at risk for $O$. volvulus infection, with most residing in rural Africa. The disease is transmitted through repeated bites by black flies of the genus Simulium. It is called "River Blindness" because the black fly that transmits the infection lives near fast-flowing streams and rivers, and the infection can result in blindness. In addition to visual impairment or blindness, onchocerciasis causes various skin diseases, including nodules under the skin or debilitating itching. Worldwide onchocerciasis is second only to trachoma as an infectious cause of blindness [1].

Various control programs were employed to stop onchocerciasis from continuing as a public health problem. The first was the Onchocerciasis Control Programme (OCP), which launched in 1974. At its peak, it covered 30 million people in 11 countries through the use of larvicide spraying of fast-flowing rivers to control black fly populations. Treatment and control of onchocerciasis as a public health problem was revolutionized in 1988 by the discovery that Mectizan ${ }^{\text {TM }}$ (ivermectin) had a potent effect on the larval stages of $O$. volvulus. It was initially believed that Mectizan $^{\mathrm{TM}}$ distribution alone could not successfully eliminate onchocerciasis in Africa, due to the widespread distribution of the infection and the intensity of transmission. However, recent data suggests that this is not the case, and that long term community wide distribution of Mectizan ${ }^{\mathrm{TM}}$ may be capable of eliminating onchocerciasis in at least some foci in Africa. This discovery has resulted in a re-focusing of the international community from an emphasis on control of onchocerciasis in Africa towards an emphasis upon possible elimination.

Unfortunately, endemic riverine communities are currently identified through ground-based epidemiological surveys. These can be difficult to conduct in remote and conflict-ridden regions of Africa, such as in Southern Sudan and the Democratic Republic of Congo. Methods to identify at-risk riverine communities from the spatial aggregation of georeferenced prolific habitats are based on fieldsampled density count values for delineating seasonal endemic onchocerciasis transmission zones. Ground-based epidemiological surveys, that overcome the time consuming, labor intensive and costly characteristics of said surveys, are urgently needed.

${ }^{{ }^{*} C o r r e s p o n d i n g ~ a u t h o r: ~ B e n j a m i n ~ J a c o b, ~ G l o b a l ~ I n f e c t i o u s ~ D i s e a s e ~ R e s e a r c h ~}$ Program, Department of Public Health, College of Public Health, University of South Florida, Tampa, Florida, USA, Tel. (813) 974-2311; Fax: (813)974-4718; E-mail: bjacob1@health.usf.edu

Received April 04, 2013; Accepted June 21, 2013; Published July 15, 2013

Citation: Jacob B, Novak RJ, Toe L, Sanfo MS, Caliskan S, et al. (2013) Unbiasing a Stochastic Endmember Interpolator Using ENVI Object-Based Classifiers and Boolean Statistics for Forecasting Canopied Simulium damnosum s.I. Larval Habitats in Burkina Faso. J Geophys Remote Sensing 2: 109. doi:10.4172/21690049.1000109

Copyright: $\odot 2013$ Jacob B, et al. This is an open-access article distributed under the terms of the Creative Commons Attribution License, which permits unrestricted use, distribution, and reproduction in any medium, provided the original author and source are credited. 
Citation: Jacob B, Novak RJ, Toe L, Sanfo MS, Caliskan S, et al. (2013) Unbiasing a Stochastic Endmember Interpolator Using ENVI Object-Based Classifiers and Boolean Statistics for Forecasting Canopied Simulium damnosum s.l. Larval Habitats in Burkina Faso. J Geophys Remote Sensing 2: 109. doi:10.4172/2169-0049.1000109

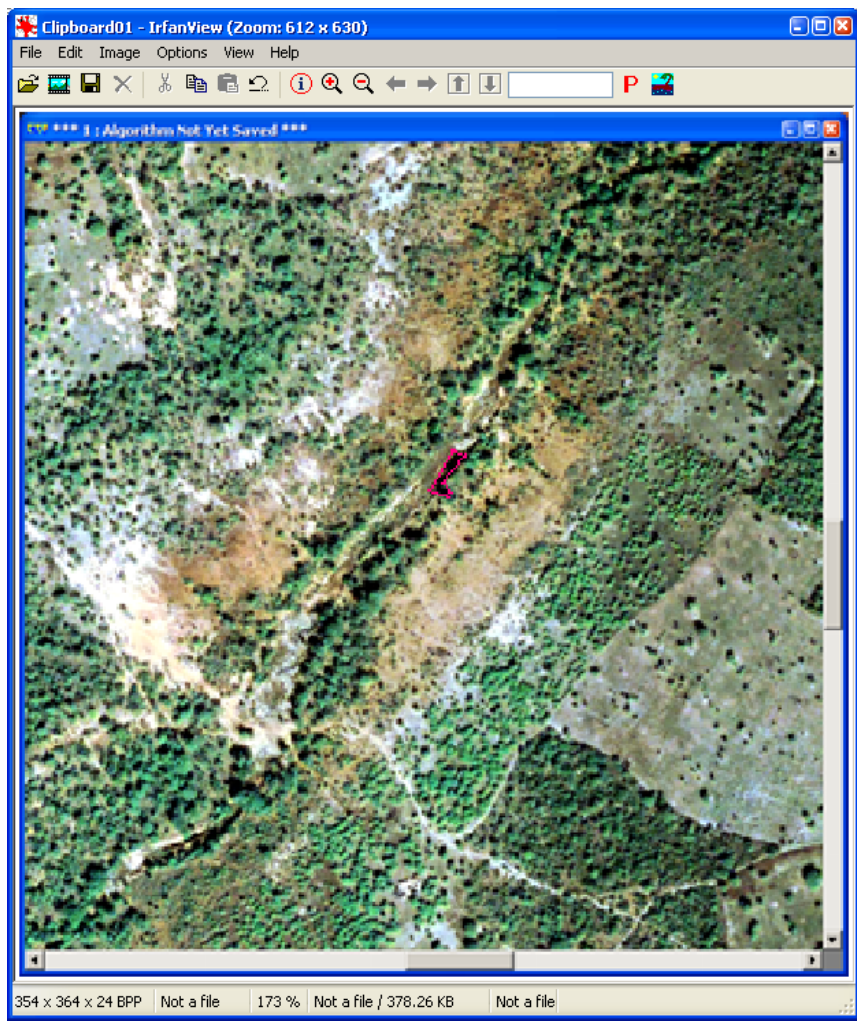

Figure 1: QuickBird base map of the canopy shaded S. damnosum s.l. riverine habitat site polygonized within an $\mathrm{ArcGIS}^{\circledast}$ database.

Treatments or habitat perturbations should be based on surveillance of count data in the most productive areas of an ecosystem [2].

Fortunately, remotely-sensed seasonal geopredictive arthropod-related infectious disease mathematical models (ranging from simple linear regression models to more complex multivariate models) have been used to link satellite data with decomposed sub-pixel radiance measurements to image endmember properties. Image endmembers (henceforth, referred to simply as endmembers) are pixel spectra that lie at the vertices of the image simplex in $n$-dimensional space. Spectra measured within the instantaneous field of view (IFOV) can then be modeled as the linear mixture of the endmembers for spectrally representing different materials within the image. The IFOV is a range of incident angles seen by a single detecting element in the focal plane [1]. The IFOV of a georeferenced larval habitat can be calculated using trigonometry: IFOV $=2 \tan ^{-1}\left(\frac{d}{2 f}\right)$ or $I F O V=2 \tan ^{-1}\left(\frac{D_{F S}}{2 f}\right)$ [1]. For small angles, less than $20^{\circ} \mathrm{C}$, which is generally the case for IFOVs associated to seasonal geopredictive vector arthropod-related aquatic larval habitat endmember endemic transmission-oriented models, the inverse tangent can be accurately approximated by $\tan ^{-1}(\mathrm{x}) \mathrm{x}$ (radians), in which case: IFOV $\mathrm{d} / \mathrm{f}$, if $\mathrm{d} / \mathrm{f}<1$. In remote sensing, one can use small angle approximations so that IFOV $=\mathrm{dx} / \mathrm{f}^{\star} \mathrm{dy} / \mathrm{f}$.dx and dy are the physical dimensions of the pixel [1]. Endmembers can then be selected from spectrally decomposed S. damnosum s.l. riverine larval habitat image pixels, measured in the field or laboratory, or even as "virtual" endmembers.

Once a satellite image is entered into an image-processing package (e.g., ENVI), interactive tools can be employed to view and analyze vectors and derive ArcGIS attributes. Encoded-radiances of Digital Numbers (DNs) in all S. damnosum s.l. riverine larval habitat spectral bands can then be transformed to reference endmembers fractions. Then, the intensity of the riverine larval habitat pixel can be digitized and recorded as a digital number (DN). This intensity value of the georeferenced riverine larval habitat can represent any measured physical quantity (e.g., solar radiance) in a given wavelength band reflected from the ground emitted infrared radiation or backscattered radar intensity. A pixel has an intensity value and a location address in the two dimensional image [1]. This value for a georeferenced seasonal-sampled S. damnosum s.l. riverine larval habitat spectral measurements are the average value for the whole ground area covered by the pixel. Due to the finite storage capacity, a DN is stored with a finite number of bits (e.g., binary digits) [1]. The number of bits determine the radiometric resolution of the riverine larval habitat image. For example, an 8bit $\mathrm{DN}$ extracted from a $S$. damnosum s.l. riverine larval habitat image ranges from 0 to 255 (i.e., $2^{8}-1$ ), while an 11 -bit $\mathrm{DN}$ ranges from 0 to 2047. The detected riverine larval habitat pixel intensity value needs to be scaled and quantized to fit within this range of values. In a radiometrically calibrated image, the actual intensity value can be derived from the pixel DN [1].

Thereafter, reflectance spectra of well spectrally-characterized $S$. damnosum s.l. larval habitat materials can be seasonally quantitated. Since endmember fractions are easier to interpret than DNs [1], a more intuitive link between the riverine larval habitat image measurements and observations in the field can be facilitated. For example, an infectious disease epidemiologist or an abatement district manager may readily understand the significance of a $S$. damnosum s.l. larval habitat pixel having $65 \%$ green vegetation and $35 \%$ soil, but may have more difficulty interpreting the equivalent band-radiance values. Alternatively, spectral classes may be defined as domains of fractions of the riverine larval habitat endmembers. The larval habitat pixel classes may be defined in a physical context, such as the amount of green vegetation, rather than on statistically defined habitat clusters. These spectral classes may be also employed as dependent variables in stochastic/deterministic interpolators (e.g., inversely distance weighted matrix) for geospatially forecasting aggregations of seasonally productive infectious disease arthropodrelated larval habitats based on field-sampled density count data. It is well known that performance of sub-pixel target detection algorithms can utilize interpolated endmembers to characterize the background and the target spatial objects in hyperspectral imagery [3]. Thus, endemic transmission zones (e.g., hypendemic, mesoendemic, hypoendemic) may be identified based on seasonal aggregations of productive S. damnosum s.l. habitats.

Although S. damnosum s.l. related spectral endmembers can be seasonally interpolated to determine onchocerciasis-related endemic transmission zones based on spatial aggregations of productive larval habitats, canopy larval habitat endmember spectra may mix the spectra with shade during the unmixing decomposition algorithmic process. As such, canopy spectral variations will not be properly simulated based on the illumination values caused by the topography of the riverine epidemiological study site and the surface texture of the sampled larval habitat. Shade-related canopy endmembers will vary based on seasonal-sampled vector arthropod-related land use land cover (LULC), vegetation type and vegetation density [1]. This spectral unquantitation of shaded endmembers can complicate accurately calculating riverine larval habitat reflectance emissivities as the upwelling radiance from 
the canopy shaded imaged materials will be integrated into a single observed spectrum. As a result of each sensor element's IFOV imaging, more than one canopy-related shaded feature attribute, extensive "mixing" of shaded larval habitat spectral components would occur. If a standard single class per habitat pixel classification is then performed, the spectral accuracy would be compromised since a shaded canopy endmember fraction of a decomposed $S$. damnosum s.l. riverine larval habitat pixel would be incorrectly classified. Endmembers are the reflectance spectra of the pure component materials that occur in the scene [1]. In the worst case scenario, mixing may produce a confusing spectral combination such that the shaded canopied riverine larval habitat endmember signature may be totally incorrect thus causing biased forecasts during interpolation.

Spectral signatures are the specific combination of emitted, reflected or absorbed electromagnetic (EM) radiation at varying wavelengths which can uniquely identify an object [1]. As such, the spectral signature of a georeferenced $S$. damnosum s.l. riverine larval habitat is a function of the incidental EM wavelength and material interaction within a specific section of the EM spectrum. The measurements can be made with various instruments, including a task spectrometer. However, the most common method is separation of the red, green, blue and Near Infrared (NIR) portion of the EM spectrum as acquired by satellite imagery. Calibrating spectral signatures under specific illumination allows an empirical correction to airborne or satellite imagery digital images [1]. Most remote sensing applications for arthropod-related infectious disease larval habitat process digital images to extract spectral signatures at each pixel and use them to divide the image in groups of similar pixels (i.e., segmentation) based on seasonal LULC types, vegetation, elevation, and others [2]. Ultimately correct matching of spectral signatures recorded by an image pixel with spectral signature of existing $S$. damnosum s.l. riverine larval habitats elements can lead to accurate endmember classification. Thereafter, by employing the endmember signature (i.e., waveband ratio of georeferenced habitat) as a dependent variable in an ArcGIS interpolation algorithm, accurate geopredictive risk maps spatially targeting endemic transmission zones based on aggregations of productive habitats and prevalence rates can be generated.

Unfortunately, identifying pure canopy shaded S. damnosum s.l. riverine larval habitats endmember fractional radiance estimates may be difficult for generating a robust interpotable signature. Canopied endmember fractional estimate accuracies are prone to residual spectral error caused by ambient (e.g., inaccurate atmospheric correction) [4], sensor-specific [e.g., insufficient Signal-toNoise Ratio (SNR) [5] and model structure input noise (e.g., nonlinear mixing) [6]. The Signal-to-noise ratio is an important measure employed when seasonally interpolating arthropod-related infectious disease larval habitat endmember spectra as it compares the level of a desired signal to the level of background noise [2]. For example, a ratio higher than 1:1 would indicate a non-normal SNR in a geopredictive canopy shade-related $S$. damnosum s.l. riverine larval habitat endmember endemic transmission-oriented model. Unfortunately, a small percentage of noise in reference endmember canopy shaded spectra can lead to disproportionally large errors in interpolated riverine larval habitat endmember fractions.

In a conventional approach, a simple spectral mixing algorithm (sSMA) can define image-wide canopy shaded endmember spectra for interpolating endemic transmission zones based on an interpolated signature for identifying unknown, unsampled, aggregations of prolific S. damnosum s.l. riverine larval habitats. For instance, the value of explanatory, time-series, sub-pixel fraction maps provided by sSMA demonstrated in the following climate change research [7-11], terrestrial ecosystem monitoring and management [12-15], precision agriculture [16-18], production monitoring [19-21], natural hazard risk assessment [22-25], forest inventories and forest health assessments [26-28], water quality assessment $[29,30]$, fresh water management [31], geological mapping [32,33], mapping of the urban environment [33-37] and extraterrestrial mapping surveys [38-40]. As such, based on an available spectral library, a standard (i.e., commonly the mean) spectrum can be defined for selected riverine larval habitat canopy endmember using an sSMA for interpolating endemic transmission zones in an epidemiological riverine study site.

sSMA techniques typically utilize a shade endmember to account for variations in spectral albedo to identify the spectral constituents of the canopied modeled spectrum [1]. In this unmixing algorithmic process, each decomposed endmember commonly is assigned a fractional abundance, such that the reflectance of the canopied modeled spectrum is determined by the sum of the reflectance of each material within a pixel multiplied by its fractional cover. The primary advantages of sSMA for $S$. damnosum s.l. riverine larval habitat endmember endemic transmission-oriented risk modeling the spectral angle would be that the technique is insensitive to differences in the albedo of the canopy shaded spectrum. Spectral angle only measures differences in spectral shape, and spectral albedo is measured by the length of each vector [1]. Although a sSMA can provide physically meaningful and plausible measures of robust endmember fraction abundances for different materials on urbanized slightly heterogeneous LULC, it may not account for the subtle spectral variations among smaller anomalous riverine-related, spectral, canopy, shaded endmembers of georeferenced S. damnosum s.l. larval habitats. The spectrally differentiated physical compositions may not, for example, properly reveal the excessive heterogeneity among the unmixed canopy shaded sub-pixel feature data attributes. The underlying assumption for linear mixing systematics would be violated since each extracted larval habitat sub-pixel of the canopy shaded surface would not be a physical mixture of multiple components weighted by surface abundance. The spectrum of the mixture would not be a linear combination of the endmember reflectance spectra. Further, situations where radiation interacts with more than one shaded subpixel S. damnosum s.l. riverine larval habitats canopied endmember component, the mixing would be rendered nonlinear. The sSMA would convert the canopied shaded larval habitat reflectance values into a single scattering albedo. Single-scattering albedo is the ratio of scattering efficiency to total extinction efficiency (which is also termed "attenuance", a sum of scattering and absorption) as defined for small-particle scattering of electromagnetic waves [1]. A singlescattering albedo of unity in a seasonal canopy, shaded, S. damnosum s.l. riverine larval habitat endmember endemic transmission-oriented risk model would imply that all particle extinction is due to scattering. Conversely, a single-scattering albedo of zero would imply that all extinction is due to absorption. Unfortunately, nonlinear functions of shade canopy spectral endmember components are easily contaminated by Additive White Gaussian Noise (AWGN) [41].

Additive white Gaussian noise is a channel model in which the impairment is a linear addition of wideband or white noise with a constant spectral density (expressed as watts per hertz of bandwidth) and a Gaussian distribution of amplitude [1]. In many occasions, noise in vector arthropod-related larval habitat digital images is found to be additive in nature with uniform power in the whole bandwidth with Gaussian probability distribution (i.e., AWGN).The AWGN is mathematically represented by,

$\eta N A W G N(t)=\eta G(t) \sigma f A W G N=f(x, y)+\eta G(x, y)$ 
Page 4 of 36

where $\eta \mathrm{G}(\mathrm{t})$ is a random variable that has a Gaussian probability distribution [1]. In a noisy, canopy, shaded, S. damnosum s.l. riverine larval habitat image, AWGN can be represented by the Gaussian distributed random noise $\eta \mathrm{G}$. For instance, when the variance of the random noise $\eta \mathrm{G}$ is very low in the riverine larval habitat image, $\eta \mathrm{G}(\mathrm{x}, \mathrm{y})$ would be zero, or very close to zero, at many sub-pixel-related canopy shade-related larval habitat endmember locations. Under such circumstances, the noisy image would be the same or very close to the original image $f(x, y)$ at these georeferenced larval habitats locations ( $\mathrm{x}, \mathrm{y})$. Fortunately, innovative techniques for denoising Gaussian noise using the concept of grouped mean and fuzzy derivatives are presented and implemented effectively in literature $[4,5]$.

A fuzzy set is a pair $(U, m)$ where $U$ is a set and $: m U \rightarrow[0,1]$. For each $\mathrm{x} \in U$ the value $m(x)$ is called the grade of membership of $x$ in $(U, m)$.

For a finite set $U=\left\{x_{1}, \ldots ., x_{n}\right\}$ the fuzzy set $(U, m)$ is often denoted by $\left\{m\left(x_{1}\right) / x_{i}, \ldots, m\left(x_{n}\right) / x_{n}\right\}[4,5]$. Many classical results are obtained as direct corollaries of propositions from fuzzy derivatives. For instance,

a fuzzy differentiable function finds many useful applications to problems of Artific al Intelligence, pattern recognition, decisionmaking, operation research and many others. In a noisy, canopy, shaded, S. damnosum s.l., riverine, larval habitat image, AWGN can be dealt with by fuzzy differentiable functions in two ways. First and foremost, the properties of fuzzy functions and their integrals for a fixed, canopy, shaded, riverine larval habitat point spectral properties can be parsimoniously seasonally quantitated. The second aspect would be to "fuzzify" the larval habitat image AWGN. Although these functions may be approximated using polynomial functions leading to construction of a polynomial post nonlinear canopy, shaded, riverine, larval habitat, mixing model, the residual forecasts would not account for fading, frequency selectivity, interference or any propagated endmember interpolated error.

Alternatively, a Bayesian image optimization method may be proposed instead to estimate the canopy, shaded, S. damnosum s.l. endmember parameters for constructing a robust, geopredictive, endemic, transmission-oriented risk model. The performance of the unmixing strategies may be evaluated by simulations conducted on synthetic or real seasonal-sampled larval habitat data. In the Bayesian spectral mixture analysis, the endmember signatures would be represented by probability density/mass functions not as constants as in the sSMA. Then, the analyses exploit the properties of a Markov random field (MRF) and the associated Gibbs equivalence theorem employing a suitably defined graph structure and a Gibbs distribution to model the partition process. Consequently, spatial consistency would be imposed on the spectral endmember partition and content of the canopy, shaded, river larval, habitat geopredictive, endmember model. The enhanced spectral mixing process would then computed a linear mixture model that is conditioned on the partition process. MRF theory is often used in conjunction with statistical decision and estimation theories so as to formulate objective functions in terms of established optimality principles [2]. Maximum a posteriori (MAP) probability is one of the most popular statistical criteria for optimality and is the most popular choice in MRF modeling for image analysis. MRFs and the MAP criterion together give rise to a MAP-MRF canopy, shaded, S. damnosum s.l. endmember framework. The MAPMRF model is advocated by Geman and Geman [42] among others [43-47]. This model enables developing algorithms for a variety of seasonal-sampled S. damnosum s.l. canopy, shaded, spectral, endmember decomposition problems systematically using rational principles, rather than relying on ad hoc heuristics. Experiments to date have been performed using scenes of HYDICE imagery to validate the residual algorithm where sSMA had been performed with and without conditioning on the partitioning process.

Although many approaches to Bayesian image segmentation have used MAP estimation in conjunction with MRF, this approach may not perform well for risk modeling canopy, shaded, S. damnosum s.l. larval habitats. In particular, exact MAP-related larval habitat estimates may not be computed as the method employs unsupervised parameter estimation of the MRF which is very difficult. Although a new method of Bayesian image segmentation have been recently proposed to address this problem, specifically by replacing the MRF model with a Multi Scale Random Field (MSRF) and the MAP estimator with a sequential MAP (SMAP) estimator, this novel estimation criteria is yet to be tested in literature.

On the other hand, the multiple endmember spectral mixture analysis (MESMA) technique may overcome the limitations of the traditional sSMA and Bayesian image segmentation analyses for spectrally extracting and decomposing georeferenced $S$. damnosum s.l. riverine larval habitats. MESMA extends sSMA by allowing the number and types of endmembers to vary on a per-pixel basis [1]. MESMA overcomes limitations of sSMA by requiring a model to meet minimum fit, fraction and residual constraints while testing multiple models for each image pixel. MESMA is typically implemented by developing a spectral library, then unmixing an image using every possible combination of two, three and four endmembers applied to each pixel. Using this approach, significantly more than four materials can be mapped across a canopy, shaded, S. damnosum s.l. riverine larval habitat image while minimizing pixel-scale fraction errors by selecting the best-fit model for each pixel.

While MESMA is a powerful approach, several factors may limit its use for accurate geopredictive endmember risk modeling canopy shaded S. damnosum s.l. riverine larval habitats. One of the major limitations is the necessity to use multiple LULCs and their endmembers to attain a target signature for interpolation. This limitation can create challenges in selecting optimal canopy, shaderelated, endemic transmission-oriented, endmembers when generating a robust, riverine, larval habitat signature. Unfortunately, MESMA typically employs hundreds of endmembers resulting in significant challenges in interpretation and computation. Since a key goal for endmember modeling canopy, shaded, S. damnosum s.l. riverine larval habitats would be to identify those spectra that are most representative of a onchocerciasis-related specific class (e.g., Precambrian rock), employing multiple endmembers would confuse the spectra. More recently, efforts were made to fine tune the analysis by selecting optimal sets of image endmembers from a spectral library prior to running MESMA. To facilitate the development of optimal spectral libraries, several techniques were recently developed. Countbased Endmember Selection (CoB), for example, were applied whereby endmembers are selected to model the greatest number of endmembers within their class. As a variation to this approach, a Count-Based Index can be used to rank endmember selection based on maximizing the canopy, shaded, S. damnosum s.l., riverine, larval habitat models selected within the correct class, while minimizing confusion with other classes. Further, average root mean square error (RMSE) endmembers are selected to produce the lowest RMSE within a S. damnosum s.l. larval habitat-related LULC class. Using Minimum Average Spectral Angle (MASA), thereafter, canopy, shaded, endmembers can optimally selected so have the lowest average spectral angle.

A promising alternative to MESMA is Automatic Monte Carlo Unmixing, as described by Asner et al. [13]. It can can be used for optimizing canopy, shade-related, endmember extraction. Using this approach, rather than selecting a single set of endmembers or predefined models, the algorithm enables the application of unmixed, 
canopy, shaded endmembers randomly selected from "bundles" of similar materials from the decomposed habitat sub-pixel data while simultaneously generating a mean and standard deviation for each fraction. Automatic Monte Carlo Unmixing (AMCU), while similar to MESMA in principle, has an advantage for spatiotemporal vector arthropod-related endmember predictive risk modeling in that it does not depend on the selection of a single set of endmembers and is readily automated. However, the fractions derived using these approaches for accurately interpolating a robust vector larval habitat endmember signature will be highly dependent on how representative library spectra are of the riverine region. Unfortunately, representative library spectra for MESMA or for an AMCU model will not account for species-specific spectral canopy endmember larval habitat differences. For example, differences in solar illumination due to topography would be of particular concern when constructing a robust S. damnosum s.l. riverine larval habitat endmember endemic transmission-oriented risk model since a partially canopy shaded surface might fall below a given RMSE threshold. Selection of spectral range and the number of bands may increase or decrease the albedo of the modelled spectra. Relationships between MESMA error metrics derived for full range spectra may thus not apply to convolved or subset spectra of immature S. damnosum s.l. canopy shaded habitats with different albedos.

Although in situ measurements may not be possible for capturing some seasonal, shaded, riverine, larval habitat, spectral, canopy endmembers, the sub-pixel spectra can be derived directly from image data or simulated using radiative transfer models. The measured spectrum may be decomposed into a combination of pure spectral signatures of each individual canopy, shaded, georeferenced, larval habitat, spatial object weighted by its areal coefficients, which would then subsequently indicate the proportion of each macroscopically pure signature in the shaded mixed pixel (i.e., mixel). Five basic principles to mitigate endmember variability have been identified in literature: (i) the use of multiple endmembers for each component in an iterative mixture analysis cycle, (ii) the selection of a subset of stable spectral features, (iii) the spectral weighting of bands, (iv) spectral signal transformations and (v) the use of radiative transfer models in a mixture analysis [1].

The use of radiative transfer models to generate canopy endmember libraries for spectral unmixing is a common approach. For example, Peddle et al. [27], used a reflectance model to create a spectral library of boreal forest canopies. Meanwhile, Eckmann et al. [22] used the MODTRAN radiative transfer model to create a spectral library of emitted canopied radiance endmembers for conducting a sub-pixel endmember analysis of wildfire properties. In line with these approaches, Somers et al. [48] proposed the Soil Modeling Mixture Analysis (SMMA). In an effort to reduce the canopied spectral effects based on soil moisture variations, a radiative transfer model for moist soil background was constructed by Lobell and Asner [49] which was implemented using SMMA. Instead of employing the soil reflectance $\mathrm{m}$ odel $\mathrm{t}$ o $\mathrm{g}$ enerate the canopy endmember library, spatiotemporal, soil moisture, content data was used to generate the corresponding spectral, soil, endmember maps. Analysis based on simulated mixtures in situ then, measured reflectance spectra of citrus canopies and soils under different moisture conditions. This illustrated that SMMA provided a dynamic and robust technique to account for spectral/ spatial and temporal variations in soil spectra. Accurate and robust sub-pixel cover fraction estimates revealed that model accuracy remained consistent over different seasonal canopy moisture scenarios. An SMMA is specifically designed to reduce seasonal soil moisture effects. It will have $n$ value in decomposing mixtures lacking a soil component within a georeferenced canopy shaded S. damnosum s.l. riverine larval habitat endmember endemic transmission-oriented risk-based seasonal model [2]. However, large-scale, soil erosion, riverine, larval habitat model factor parameterization and quantification is difficult due to the costs, labor and time involved. Among the soil erosion parameters, the vegetative cover or $\mathrm{C}$ factor has been one of the most difficult to estimate over broad geographic areas. The $\mathrm{C}$ factor represents the effects of vegetation canopy and ground covers in reducing soil loss [1].

Although many endmember extraction algorithms can identify distinctive shaded canopy pixels from image datasets as endmember signatures owing to mathematical tractability, most methods may not spectrally distinguish specific photosynthetic, canopy, shade-related, S. damnosum s.l. larval habitats endmember spectral covariates. For example, without properly quantitating Top-Of-Canopy (TOC) reflectance, a predictive endemic transmission oriented risk model may render biased estimators. The TOC spectral reflectance refers to the portion of the incident solar radiation reflected by the surface in a given waveband without atmospheric interferences [2]. Further, accuracy of vegetation cover fractions computed with only one spectral unmixing analysis may not spectrally quantitate variation in canopy structure and biochemistry especially when a single riverine larval habitat endmembers represents TOC reflectance. Theoretically, TOC reflectance should be measured for every given sun direction and for every given viewing direction for accurately spectrally quantitating seasonal $S$. damnosum s.l. riverine larval habitat canopy shaded endmembers. To do so, it would be necessary to pre-process the remote sensing data to obtain robust TOC reflectance before applying physically-based model inversion techniques to robustly interpolate the shaded riverine larval habitat signature. Advantages of this type of approach for geopredictive, autoregressive, canopied, endmember, S. damnosum s.l., risk modeling for identifying endemic transmission-oriented zones are that no atmospheric correction would be required and that atmospheric, adjacency, topography and surface directional effects would be directly and more accurately included in the model residual forecasts.

Spectrally quantitated TOC canopy radiance data has been employed to estimate explanatory, predictor variables using coupled canopy-atmosphere radiative transfer model. For example, Somers [48] applied both TOC approaches to three Norway spruce stands in Eastern Czech Republic. The authors constructed a soil-leaf-canopy (SLC) model and a MODTRAN4 atmosphere model. In the TOC approach, the physical coupling between canopy and atmosphere was performed using a generic method. This method is based on the 4 -stream radiative transfer theory which enabled full application of the directional reflectance components provided by the SLC model. The authors employed a local sensitivity analysis and singular value decomposition (SVD) to determine which predictor variables could be estimated, namely: canopy cover, fraction of bark, needle chlorophyll and dry matter content. Singular Value Decomposition can effectively address canopy spectral variance and deviation since the spatial neighborhood (SN) can be eliminated by selecting the average of the pixels in pure SNs as endmember candidates, while spectral deviation among different areas in the image can be eliminated by clustering the endmember candidates [2]. In addition, a graph theory based spatial refinement algorithm can be proposed by SVD to reduce the number of endmember candidates, which can save a lot computation in operationalizing subsequent clustering steps. For example, in Somner [48] the TOC approaches resulted in different sets of clustered estimates that had comparable performance. As such, the TOC approach could be preferred for robustly constructing 
geopredictive, autoregressive, seasonal, $S$. damnosum s.l., endmember, riverine, larval habitat, endemic, transmission-oriented, risk models.

Traditionally, the TOC spectral extraction method would utilize a minimum of three runs of an atmosphere model for quantitating Lambertian surfaces, thus avoiding running the riverine larval habitat endemic transmission-oriented risk model for each new simulation. Lambertian reflectance is the property that defines an ideal diffusely reflecting surface, whereby, the apparent brightness of such a surface to an observer is the same regardless of the observer's angle of view [1]. However, the TOC approach is only optimal in LULC that is flat and homogeneous [19]. Thus, the capacities of the TOC approach alone would not be suitable for forecasting seasonal, georeferenced, endemic, transmission zones based on aggregations of spectral/spatial $S$. damnosum s.l. due to excessive meandering and elevation shifts in the riverine pathways [2].

One relatively simple approach to quantiate seasonal riverine meandering may be to estimate river stage and discharge based on satellite-measured inundation areas and TOC. Although remote sensing of river stage and discharge provides promising alternative approach for monitoring watersheds, it may be more difficult to capture canopied, spectral/spatial, canopy, shaded, S. damsnoum s.l., endmember, related data when applying unmixing algorithms. While it is simple, there are still problems associated with applicability of this approach for seasonal, geopredictive, canopy, shaded, S. damnosum s.l., riverine, larval habitat, endmember, endemic, transmissionoriented, risk modeling. For example, the consruction of inundation area-river stage relationship (IARSR) curves without ground-based measurements results in the georeferenced data.

Recently, georeferenced data. Pan and Nichols [50] demonstrated the feasibility of a TOC Digital Elevation Model (DEM) to construct the inundation area - river stage relationship IARSR curves. A DEM is a digital representation of ground surface topography usually represented as a raster or as a triangular irregular network [2] which has been shown to generate accurate predictor variables associated to prolific vector arthropod-related habitats based on spatiotemporal field-sampled count data [1,47]. Generating the IARSR curves using a robust DEM may enable accurate identification of endmember interpolated canopy, shaded, georeferenced, S. damnosum s.l., riverine, larval habitats. It is important to understand that spectral variability, from one riverine, larval habitat image to another in a time series is based on illumination differences associated with variations in solar elevation and azimuth and local incidence angle [2]. Thus, although variations in illumination within the riverine larval habitat images may be caused by canopied macrotopography, it may be removed if a satisfactory photometric function is combined with a DEM. Riverine larval habitats would then define a DEM polygon covering the river cross section when the IARSR is constructed in ArcGIS. The width of the polygon would have to be wide enough to cover both riverbanks as well as a portion of the floodplain on both sides since riverine larval habitats can occur randomly [47]. There is also a requirement to identify the minimum elevation $\left(z_{o}\right)$ inside the georeferenced, canopy, shaded, S. damnosum s.l., riverine, larval habitat polygon. This minimum elevation would actually represent the water surface elevation inside the polygon. All connected pixels with the same elevation would be labeled as zo inside the polygon encompassing the canopy, shaded, riverine, larval habitat which then would then form the inundation area $\left(A_{0}\right)$. On the inundation area - river stage plane, the point $\left(A_{o}, z_{o}\right)$ would then represent the lower limit of the IARSR curve in the riverine larval habitat DEM. Optimally, the next step would be to employ an interative loop to forecast multiple, biophysical, geoferenced, larval habitats points based on the IARSR curves. The geographically predicted larval habitat points would then be all the sampled points above the lower limit point $\left(A_{0}, z_{0}\right)$. Further, by artificially' raising the water level and then employing the hypothetical water level to determine the associated inundation area, more sample, forecasted, larval habitat points above $\left(A_{o^{\prime}} z_{0}\right)$ could be identified. Regardless of the type of unmixing algorithm applied, the river stagedischarge rating (SDR) curve at any ungauged, epidemiological study site associated with a georeferenced S. damnosum s.l. larval habitat would be accurately spectrally/spatially quantitated in a canopied endmember riverine larval habitat geopredictive risk map targeting seasonal endemic transmission zones based on aggregations of productive larval habitats. Thereafter, the quantitated IARSR curves could be fit into a smooth spline curve to summarize any residual predictive variance error coefficients in the canopy, shaded, riverine, larval habitat DEM.

The smoothing spline is a method of smoothing using a spline function for fitting a smooth curve to a set of noisy vector larval habitat observations [1]. A spline is a sufficiently smooth polynomial function that is piecewise-defined, and possesses a high degree of smoothness at the places where the polynomial pieces connect (i.e., knots) [2]. By seasonally interpolating geomorphological terrainrelated covariates and IARSR curves associated to a georeferenced $S$. damnosum s.l. riverine larval habitat epidemiological study site capture point, seasonal unsampled, unknown, habitats may be identified. Spline interpolation is often employed for polynomial interpolations as it yields similar results, even when using low-degree splines to determine higher degree polynomials while avoiding instability due to Runge's phenomenon. In the mathematical field of numerical analysis, Runge's phenomenon is a problem of oscillation at the edges of an interval that occurs when using polynomial interpolation with polynomials of high degree [2]. This polynomial canopy, shaded, S. damnosum s.l., riverine, larval habitat, endmember interpolation would also form the basis for algorithms for constructing numerical quadratures and numerical ordinary differential equations ODE.

An ODE is a differential equation in which the unknown function (e.g., the georefernced, canopy, shaded, S. damnosum s.l., endmember fractionally decomposed dependent variable is a function of a single independent variable (i.e., presence of floating vegetation) [51]. In riverine larval habitat endemic transmission-oriented risk modeling, an ODE would be equivalent to an equation containing a function of one independent variable and its derivatives. There are many general forms an ODE can take in the riverine predictive vector arthropod related endemic transmission-oriented spectral endmember risk model. Commonly, the derivatives in a multivariate, vector arthropodrelated, larval habitat, risk model would be ordinary because partial derivatives only apply to functions of many independent variables [1,47]. A partial differential equation (PDE) is a differential equation that contains unknown multivariable functions and their partial derivatives. In contrast to ODEs, it deals exclusively with functions of a single variable and their derivatives. PDEs are used to formulate problems involving functions of several variables, and are either solved by hand, or used to create a relevant computer model [2]. However, the subject of ODEs is a sophisticated one for seasonal, canopy, shaded, S. damnosum s.l., larval habitat, endmember, endemic, transmission-oriented, risk modeling. This sophistication can even be more complex with partial differential equations primarily due to the various forms of ODE for integration in a canopy, shaded, larval habitat, geopredictive, endmember, time series, rick model. Linear differential equations are ones with solutions that can be added and multiplied by coefficients [3]. The theory of linear differential equations is well-defined and understood, and exact closed form solution can be 
easily obtained [2]. By contrast, in a seasonal, canopy, shaded, $S$. damnosum s.l., riverine, larval habitat-related, ODEs will not have additive solutions and thus would be non-linear. Rigorously quantitating seasonal forecasted solutions would be much more statically intricate as it would be impossible to represent any linear residual forecasts in a riverine larval habitat model by elementary functions in closed form rather the exact or "analytic" solutions would be in series or in an integral form in the residual forecasts targeting the endemic transmission zones and other georeferenced LULC explanatory covariates.

Seasonal canopied endmember variability may then be incorporated into the mixture analysis by representing each canopy, shaded, S. damnosum s.l., riverine, larval habitat endmember by a set or bundle of non-linear spectra. Applied to remotely sensed images, bundle unmixing produces maximum and minimum fraction images bounding the correct cover fractions and specifying error due to canopy endmember variability using spectrally contrasted seasonally quantitated TOC-related DEM sub-pixel data [2]. Because of the natural anisotropy of the riverine surface, the value of a georeferenced, canopy, shaded, S. damnosum s.l., riverine, larval habitat reflectance measurement would then be highly dependent on the illumination and viewing angular conditions and accurate spectral quantitation of the seasonal, canopied, riverine, larval habitat, canopy-shaded, endmembers. Comparing and using jointly successive unmixing algorithmic observations may then normalize the canopied, riverine, larval habitat, endmember measurements into a viable angular configuration for detecting spectrally contrasting, shaded, canopy explanatory covariates.

Spectral-based endmember extraction techniques for canopy, shaded, S. damnosum s.l., riverine, larval habitats methods may hinge on the ability to robustly discriminate between canopy pixels based on spectral characteristics retrieved from distinct high spectral contrast feature attributes (e.g., white turbid water components and Black Precambrian rock). Minimal unique low spectral contrast [e.g., within-canopy floating and dead vegetation] may be required to capture and quantitate photosynthetic-related explanatory covariates (i.e., TOC) employing more spectrally sensitive algorithms. Spectral contrast is dependent on the endmember assemblage, such that as the assemblage changes so does the "relative" spectral contrast of each endmember to all other endmembers [2]. It may then be impossible for a canopy, shaded, S. damnosum s.l., riverine, larval habitat endmember to compare low spectral contrast with respect to the full image, while simultaneously having high spectral contrast within a subset of the image. Although in situ measurements may not remotely capture some district, seasonal, S. damnosum s.l., riverine, larval habitat data, the spectrally distinct canopied spectral endmembers may be derived directly from the image data or simulated using Spatial-Spectral Endmember Extraction (SSEE) models in conjunction with a radative transfer model and a DEM.

Spatial-Spectral Endmember Extraction tools for constructing a robust spectral robust vector arthropod-related risk model would work by analyzing a seasonal riverine larval habitat scene in subsets whereby, a slight increase in the canopied spectral contrast measurements would improve the potential for canopy-related endmember to be selected. The proposed method would first perform joint spatial-spectral pixel characterization via extended geomorphological transformations from the TOC-related DEM. Then it would automatically extract pure canopy, shaded, S. damnosum s.l., riverine, larval habitat endmembers for robust signature generation by employing volume optimization and convex geometric concepts. The radiative transfer model constraints would improve the relative spectral contrast of the canopy endmember spectra such that they would have minimal unique spectral information. Therefore, the potential for inconspicuous spectrally subtle canopy shaded, endmembers related to a georeferenced riverine larval habitat to be selected could be significantly improved. Thereafter, employing the SSEE, the spectral/ spatial characteristics of decomposed S. damnosum s.l. riverine larval habitat image pixels may increase the relative spectral contrast between spectrally similar endmembers and the spatially independent canopy shaded endmembers. The SSEE algorithm would operate by searching a canopy shaded $S$. damnosum s.l. riverine larval habitat image with a local search window centered on each canopied pixel vector. Then the SVD transform would be applied to determine a set of eigenvectors that describe the canopy spectral variance in the window or partition. The entire shade canopied image is projected onto the previously extracted eigenvectors to determine a set of candidate riverine, larval habitat, canopy, shade-related, endmember pixels [2]. Commonly the SSEE methodology would make use of projecting the SVD-oriented data volume onto vectors to derive a set of candidate endmember pixels. Spatial constraints would then be employed to combine spectrally similar canopied, candidate, canopy, shaded, S. damnosum s.l., endmember pixels by testing each canopy candidate pixel vector, while subsequently maintaining spectral homogeneity in all other non-canopied shade-related, pixel vectors. Projection of the full canopy shaded S. damnosum s.l. riverine, larval habitat image dataset onto locally defined vectors determines a set of robust canopied candidate endmember pixels while imposing the spatial constraints for parsimoniously quantitating spectrally distinct and non-distinct endmembers.

Additionally, SSEE tools would account for both anomalous and normal canopy endmember. By developing simplex projection and simplex complementary projection for all sub-meter resolution pixels projected into a simplex all normalized, canopy, shaded, S. damnosum s.l., riverine, larval habitat, endmember would then be quantized. In order to geopredictively model arthropod-related infectious disease larval, habitat endmember signatures, they must be derived from submeter resolution data (e.g. (QuickBird) image data at $0.61 \mathrm{~m}$ pixel resolution with minimal classification error. Anomalous canopied endmembers can then be identified iteratively by utilizing the $l^{\circ} 2$ norm to find the maximum simplex complementary projection [47]. Further, in order to determine how many anomalous canopy endmembers extracted from the larval habitat image exist, a novel Residual Noise Probability-based algorithm can also be proposed by elegantly utilizing a canopy, shaded, S. damnosum s.l., riverine, larval habitat, spatial-purity maps constructed with SSEE tools. Experimental results on both synthetic and real datasets have demonstrated that simplex projection errors can be significantly reduced by identifying both anomalous and normal canopy endmembers using a SSEE algorithm [1]. For example,the SSEE method outperformed other widely used approaches in the analysis of a real hyperspectral derived LULC scene collected by the NASA's Airborne Visible Infra-Red Imaging Spectrometer (AVIRIS) [19]. Unfortunately, seasonal, georeferenced, canopy, shaded, S. damnosum s.l., riverine, larval habitat, SSEE-derived vectors can only be retained for each subset region if they explain a significant percentage of the sub-meter resolution canopied spectral variance. This reduces the possibility of retaining seasonally oriented vectors related to noise in the unmixing algorithm by selecting and eliminating canopied riverine larval habitat pixels that are noisy.

Mixed S. damnosum s.l. riverine larval habitat sub-meter resolution canopied pixel signals may then be robustly, parsimoniously modeled using either a linear (LMM) or nonlinear mixture model (NLMM). A mixture model then optimally convert the 
image DN's to numerical fractions of a few canopy, shaded, larval habitat endmember. The question of whether linear or nonlinear processes dominate spectral signatures of canopied, shaded, seasonal, vector arthropod-related, larval habitat pixel is still an unresolved matter. As a rule of thumb, linear mixing is associated to shade canopied mixtures for which the pixel components appear in spatially segregated patterns. However, since the spectral, canopy, shaded, pixel components in an S. damnosum s.l. riverine larval habitat are in intimate association, light typically will interact with more than one photosynthetic component as the canopy radiance would then be scattered, and the mixing systematics would be highly nonlinear. The underlying physical assumption of a LMM for decomposing and interpolating canopy, shaded, S. damnosum s.l., riverine, larval habitat pixel would be that each incident photon interacts with a single pixel component only. A canopied riverine larval habitat pixel signal $(r)$ can then be described employing a linear combination of pure spectral signatures and their constituent components (i.e., canopy-related, shaded endmembers weighted by their sub-pixel fractional cover $r=M f$ $+\varepsilon r=M f+\varepsilon$ (1.1) [2]. In equation (1.1) $M$ would be a matrix in which each column corresponding to the spectral signal of a specific $S$. damnosum s.l., riverine, larval habitat, canopy, shaded, endmember, $f$ would be a column vector $\left[f_{1}, \ldots, f m\right]^{\mathrm{T}}$ denoting the cover fractions occupied by each of the $m$ endmembers in the decomposed pixel and $\varepsilon$ would be the portion of the spectrum that cannot be modeled using the endmembers.

NLMM's may also account for a nonlinear mixing process, which is a product of multiple scattering between at least two materials in the IFOV. Based on the radiosity theory described by Borel and Gerstl [6], nonlinear mixing effects can be modeled explicitly by including additional endmembers to the LMM. Each accounts for a characteristic interaction among ground objects, such as river-based, canopied, shaded, georeferenced, S. damnosum s.l., larval habitats. Employing a training dataset, the mathematical relationships between input (i.e., spectral data) and output data (i.e., classes of interest) from a georeferenced, seasonal-sampled, riverine, larval habitat may then be automatically calculated using advanced computer modeling approaches such as Artificial Neural Network. (ANNs).

Artificial Neural Networks for geopredictive, canopy, shaded, $S$. damnosum s.l., riverine, larval habitat risk modeling essentially mathematical models defining a function $f: X \rightarrow Y$ or a forecasted distribution of specific endemic transmission-oriented explanatory covariate over X or both $\mathrm{X}$ and Y. Sometimes these models are also intimately associated with a particular learning algorithm or learning rule. A common use of an ANN model is for defining a class of functions, whereby members of each class are obtained by varying parameters, connection weights or specifics of the architecture, such as the number of neurons or their connectivity. The word network in the term 'artificial neural network' refers to the inter-connections between the neurons in the different layers of each system [2]. Optimally, an example system for an ANN-related, seasonal, canopy, shaded, geopredictive, S. damnosum s.l., riverine, larval habitat, endmember, endemic, transmission-oriented, risk model have three layers. The first layer would have input neurons, which would send the seasonal-sampled data via synapses to the second layer of neurons, and then via more synapses to the third layer of output neurons. More complex systems will have more layers of neurons with some having increased layers of input neurons and output neurons. The synapses can then store the georeferenced, seasonal-sampled, canopy-related, S. damnosum s.l., endmember, endemic, transmission-oriented, parameter estimators (i.e., spectral "weights") while simultaneously manipulating the data to generate linear and non-linear residual forecasts. The linear residual forecasts. The ANN-related, endemic, transmission-oriented, risk model can then be defined by three types of information-based estimators: 1) the interconnection pattern between different layers of neurons, 2) the learning process for updating the sampled weights based on data retrieved from the interconnections and, 3) the activation function that converts a neuron's weighted input to its output activation.

\section{Mathematically, a neuron's network function $f(x)$ is defined as a} composition of other functions $g_{i}(x)$, which can further be defined as a composition of other functions [41]. In a seasonal-sampled, robust, canopy, shaded, S. damnosum s.l., endmember, endemic transmission oriented risk model this function can be conveniently represented as a network structure with arrows depicting the dependencies between the sampled observational georeferenced canopy, shaded, explanatory, predictor, variables. A widely used type of composition used for seasonal endmember risk mapping is the nonlinear weighted sum, where $f(x)=K\left(\sum_{i} w_{i} g_{i}(x)\right)$ and where $\mathrm{K}$ (i.e., the activation function) is some predefined function such as the hyperbolic tangent. It may be convenient to refer to a collection of functions in a georeferenced $S$. damnosum s.l. riverine larval habitat geopredictive, endemic, transmission-oriented, risk model [i.e., $g_{i}$ ] as simply a vector $g=\left(g_{1}, g_{2}, \ldots, g_{n}\right)$ depending of the residual forecast function. In supervised learning, for example, given a set of larval habitat pairs [e.g., $(x, y), x \in X, y \in Y$ ] from a seasonal empirical sampled dataset of canopy shaded $S$. damnosum s.l. larval habitat explanatory covariate, the aim would be to find a function $f: X \rightarrow Y$ in the allowed class of functions. In other words, to infer the endemic transmission-oriented risk mapping implied by the sampled canopy shaded data, the function would have to be related to the mismatch between the risk model uncertainty estimates and the residual forecast quantitated uncertainties to determine if the sampled data implicitly contains prior knowledge about the problem domain. A commonly used observational uncertainty-based residual predictor is the meansquared error (MSE), which tries to minimize the average squared error between the network's output, $f(x)$, and the target value y over all the example pairs. As such, if an infectious disease epidemiologist and/or an abatement district-level [1] manager tries employing gradient descent for class of neural networks (i.e., multilayer perceptrons), for spectrally quantitating a canopied, shaded, $S$. damnosum s.l., riverine, larval habitat, endmember endemic, transmission-oriented, risk model, they would derive the common and well-known back propagation algorithm for training neural networks. Tasks that fall within the paradigm of supervised learning are pattern recognition (i.e., canopied, shaded, S. damnosum s.l., riverine, larval habitat, endmember classification) as qualitatively spatiotemporally quantitated by regression estimation (i.e., function approximation of the larval habitat residual forecasts). The supervised learning paradigm would also be equally applicable to sequential, seasonal, geopredictive, canopied, riverine, larval habitat, endemic, transmission-oriented explanatory, stochastically/deterministically, interpolated, data variables.

The goal of any supervised learning algorithm for qualitative seasonal, geopredictive, autoregressive, S. damnosum s.l., larval habitat, endemic, transmission-oriented, risk mapping would be to find a function that best maps a set of inputs to its correct output. An example would be a simple classification task, where the input is an image of a georeferenced, riverine, canopy, shaded, larval habitat, and the correct output would be the seasonal larval density count. Some input and output patterns can be easily learned by single-layer neural networks (i.e. perceptrons) [2]. However, these single-layer perceptrons cannot quantitate relatively simple spatial trend patterns, such as endemic transmission oriented larval habitat data feature 
attributes that are not linearly separable. Ascribing labels to a submeter resolution riverine epidemiological study site image for endmember risk mapping georeferenced, seasonal-sampled, canopy, shaded, S. damnosum s.l., riverine, larval habitat data may help quantitate the spectral sub-pixel covariates. An infectious disease epidemiologist or a district-level abatement manager may then classify a sub-resolution image of a georeferenced, canopy, shaded, $S$. damnosum s.l., riverine, larval habitat thus, recognizing certain features such as the number of Precambrian rock, turbidity, the size of the habitat and other covariates.

A single-layer neural network would have to learn a function that outputs a label solely using the intensity of the shaded, canopied pixels in the sub-meter resolution image. Unfortunately, there is no way for the network to learn any abstract features of the input since it is limited to having only one layer. A multi-layered network can overcome this limitation as it can create internal representations and learn different features in each layer. The first layer may then be responsible for learning the orientations of lines employing the inputs from the individual canopied pixels in the riverine study site image. The second layer may combine the georeferenced spectral feature attributes learned in the first layer and subsequently learn to identify simple shapes such as polygons. Each higher layer would add more and more canopied, shaded, S. damnosum s.l., riverine, larval habitat sub-pixel abstract features. Next, they can be used to classify the georeferenced, canopied, endmember, oriented, larval habitat image. Each layer would then include spatial patterns from the layer below including internal representations that are independent of outside inputs, rendering multi-layered, canopy, shaded, riverine, larval habitat, endmember networks. The goal and motivation for developing a custom back propagation algorithm with seasonalsampled, geopredictive, explanatory, S. damnosum s.l., riverine, larval habitat, canopy, endmember, endemic, transmission-oriented, riskbased, covariate, coefficients, would then be to find a way to train multi-layered neural networks, such that it can learn the appropriate internal habitat representations.

There are three modes of learning to choose from: on-line, batch and stochastic for explanatory time series endmember S. damnsoum s.l. riverine larval habitat modeling. In on-line and stochastic learning, each propagation would be followed immediately by a weight update in a riverine, larval habitat, canopy, endmember, endemic, transmission-oriented, risk- based model. In batch learning, much propagation occurs before updating the weights [1]. Batch learning requires more memory capacity, but on-line and stochastic learning require more updates [1]. On-line learning is used for dynamic environments that provide a continuous stream of new patterns [2]. Stochastic and batch learning both could robustly regress an empirical dataset of S. damnosum s.l. riverine larval habitat training set of static patterns. Stochastic models, for example, go through the sampled dataset in a random order to reduce its chances of getting stuck in local minima. Stochastic learning is also much faster than batch learning since weights are updated immediately after each propagation [2]. Yet, batch learning would yield a much more stable descent to a local minima since each update would be performed based on all patterns in the canopy, shaded, riverine, larval habitat, endmember, endemic, transmission-oriented risk model.

In unsupervised learning, some riverine, canopy, shaded, $S$. damnosum s.l., riverine, larval habitat data $\mathrm{x}$ would be provided and thus the function can be minimized such that any function of the seasonal-sampled data $\mathrm{x}$ could be correlated with network's output, $f$. The function would be dependent on the task (i.e., geopredictively, endmember, risk modeling productive canopy, shaded, S. damnosum s.l., riverine, larval habitats) and the priori assumptions for parsimoniously quantitating the implicit properties of the residual forecasts, its parameters and the observed variables. As a trivial example, consider the geosampled $S$. damnosum s.l. riverine larval habitat model $f(x)=a$, where there is a constant within the equation $C=E\left[(x-(f x))^{2}\right]$. Minimizing the model output would then render a value of $a$ that it is equal to the mean of the seasonal-sampled, larval habitat data. The function of the residual forecasts targeting the canopied, riverine, canopied, endmembers can be much more complicated. For example, the form of the output may depend on the linearized parameter estimator used application in the larval habitat geopredictive risk model. As such, compression could be related to the mutual information between $x$ and $f(x)$, in statistical seasonal, geopredictive, canopied, S. damnosum s.l., riverine, larval habitat, endmember, endemic, transmission-oriented, risk modeling, since the spectral estimates would be related to the posterior probability of the residual forecasts. Note that the residual forecasted quantities (e.g., georeferenced, hyper endmember, endemic, transmission-oriented, larval habitat covariates) would be maximized rather than minimized. Alternatively, tasks that fall within the paradigm of unsupervised learning would be in general estimation problems. This would include applications like clustering and the estimation of statistical distributions of the georeferenced, larval habitat, endmember, endemic, transmission-oriented, risk variables while including the compression and filtering of the residual forecasts. Depicting such decomposition within dependencies between sampled, canopy, shaded, riverine, larval habitat, risk variables can be interpreted in two ways thereaf ter employing a ANN dependency graph. The first view would be the functional view: the input $x$ is transformed into a 3dimensional vector $(h)$, which would then be transformed into a 2 dimensional vector $(g)$, and then into a single predictor $(f)$. This view would be most commonly encountered during the context of error optimization of the georeferenced, canopy, shaded, riverine, larval habitat model residual forecasts. The second view would be the probabilistic view: the riverine, larval habitat, canopy, shaded, endmember, model variable $F=f(G)$ would be dependent upon the random variable $G=g(H)$, which would then, in turn, be dependent upon $H=h(X)$. This output would then be dependent upon the random variable $\mathrm{X}$ in a robust, $\mathrm{S}$. damnosum s.l., riverine, larval habitat, canopy, shaded, endmember, endemic, transmission-oriented model. However this endmember modeling effort could only be in the context of a post graphical, spectral, optimization, mixture analyses.

Commonly employed endmember optimization techniques are Gramm-Schmidt Orthogonalization, Quadratic Programming, maximum-likelihood or least square regression analyses. These techniques can determine the component parts of canopy, shaderelated, S. damnosum s.l., riverine, larval habitat, endmembers by predicting the proportion of a pixel that belongs to a particular class or spatial feature attribute based on the characteristics of its canopy shade-related endmembers. However, there may be other misspecifications to effectively address canopy, endmember variability issue in the optimization techniques for seasonally interpolating a canopied, endemic, transmission-oriented, georeferenced, $S$. damnosum s.l., riverine, larval habitat signature. For instance, selecting canopy endmember for natural systems such as riverine environments can be exceedingly difficult as potential surface-related, canopied, endmembers (e.g., Precambrian rock) may not occur in patches larger than the image resolution.

Additionally, inherent variability in riverine areas (e.g., rainfall, soil minerals, growing cycle phase) makes it difficult to match image canopied endmembers with actual pixel composition on the ground. Further, errors in solar irradiance and atmospheric transmittance may affect spectral seasonal quantitation of diffuse surface reflectivity power (i.e., albedo), which, in turn can effect robustness of sub-meter 
resolution, sub-pixel S. damnosums.l. riverine larval habitat canopy, shaded, endmember signatures. These spectral uncertainties will render misspecifications in residual forecasts targeting explanatory, endmember, endemic, transmission-oriented, risk-based, covariate coefficients for interpolating unmixed risk patterns based on canopyoriented, spatiotemporal, riverine, larval habitat georeferenced data [2]. Although the attenuating properties of the atmosphere can be presented in a form that permits the calculation of direct, diffuse and global spectral solar irradiance emitted from a georeferenced, canopied, S. damnosum s.l., riverine, larval habitat, the terrestrial irradiance values rendered from the canopy surface solar irradiance (SSI) calculations would not be able to provide accurate seasonal, spectrally, decomposed, canopy-related, endmember reflectance values. A particularly important component in endmember, empirical-seasonal, arthropod-related, infectious disease risk modeling is the SSI, (i.e., surface insolation), (e.g., the amount of down-welling solar energy incident on the larval habitat horizontal surface) which governs the flux of solar energy at the interface between the atmosphere and the other components of the climate system [1].

However, the potential of seasonal climatic effects of a sustained decrease or increase in SSI associated to a canopy, shade-related, $S$. damnosum s.l., endmember, geopredictive, endemic, transmissionoriented, risk model would require extensive investigation of additional atmospheric factors, (e.g., long-wave radiative effects of any associated riverine cloud variations). As such, the possible spectral endmember residual effects derived from regressed explanatory, photosynthetic, explanatory covariate coefficient measurement indicator values would be more direct although, they would differ by geographic location. In West African equatorial regions, for example, where the black-fly vector is known to flourish, riverine ecosystems are sufficiently illuminated and canopy photosynthesis may be likely limited by $\mathrm{CO}_{2}$ not by radiation. As such, canopy, shaded endmember photosynthesis rates derived from unmixing a georeferenced $S$. damnosum s.l. riverine larval habitat sub-meter resolution image pixel may be measured throughout the day and thus can be expressed as a function of photosynthetically active radiation $(\mathrm{PAR})$ and $\left[\mathrm{CO}_{2}\right]$.

Photosynthetically Active Radiation is the amount of light available for photosynthesis, which is light in the 400 to 700 nanometer wavelength range [2]. PAR changes seasonally and varies depending on the latitude and time of day. Levels are greatest during the summer at mid-day. Factors that reduce the amount of PAR available to vector arthropod-related habitat and surrounding plants include anything that reduces sunlight, such as cloud cover and shading [1]. In such circumstances, the diffuse reflected light would penetrate deeper into the canopied, georeferenced, S. damnosum s.l., larval habitat reaching a larger fraction of the biomass. Theoretically, in an ArcGIS seasonally constructed geopredictive autoregressive canopy, shade-related, $S$. damnosum s.l., riverine, larval habitat, georeferenced, endmember, risk model, the accumulation of photosynthates per leaf area unit would be calculated using a numerical integration. Thereafter, photosynthetic explanatory covariates coefficients associated to prolific seasonal-sampled riverine larval habitats can be determined employing spectrally-oriented parameters such as the leaf area index (LAI) and the extinction coefficient the percentage of PAR transmittance through the leaf and the diffuse component of incident PAR. Leaf Area Index (LAI) is a dimensionless quantity that characterizes plant canopies Leaf Area Index. It is defined as the onesided green leaf area per unit ground surface area (i.e., $\mathrm{LAI}=\mathrm{leaf}$ area/ ground area, $\mathrm{m}^{2} / \mathrm{m}^{2}$ ) in broadleafcanopies [1].

Additionally, PAR action spectrum for a spatial object such as a canopied S. damnosum s.l. riverine habitat can be seasonally quantitated for deriving absorption spectra for chlorophyll-A, chlorophyll-B and carotenoids. Carotenoids are organic pigments found in the chloroplasts and chromoplasts of plants and other photosynthetic organisms like algae some bacteria and some fungi [2]. Chlorophyll, the most abundant plant pigment, is most efficient in capturing red and blue light [3]. Further, since accessory pigments, such as carotenes and xanthophylls, would harvest some green light and pass it on to the photosynthetic process, the canopy, shaded, $S$. damnosum s.l., riverine habitat reflected seasonal green wavelengths may be quantitated using seasonal PAR measurements. PAR sensors stationed at various levels of the habitat canopy can measure the pattern of PAR availability and utilization. Photosynthetic rate in the georeferenced riverine larval habitat and related seasonal-sampled parameter estimators can then be measured non-destructively using a robust photosynthesis system whereby, instruments measure seasonal PAR rates while controlling PAR at set intensities. PAR measurements, for example, may be used to calculate the euphotic depth in the riverine central pathways and their related tributaries. PAR is normally quantified as $\mu / \mathrm{mol} /$ photons $/ \mathrm{m}^{2} /$ second, which is a measure of the photosynthetic photon flux (area) density, or PPFD. PAR can also be expressed in energy units (irradiance, $\mathrm{W} / \mathrm{m}^{2}$ ). W/ $\mathrm{m}^{2}$ measurements are important in energy balance considerations for photosynthetic organisms [1]. Because photosynthesis is a quantum process, PPFD may be also employed in linear and non-linear regression-based models for seasonally predicting canopy, shaded, photosynthetic, spectral, endmember explanatory covariate coefficients. These measurements may then be employed in spatiotemporal, linear, canopy, shade-related, S. damnosum s.l., riverine, larval habitat, endemic, transmission-oriented, model as independent variables for determining statistical significance of each sampled covariate coefficient estimates.

Increasing atmospheric, seasonal-sampled $\mathrm{CO}_{2}$ concentrations may likely be accompanied by increasing canopy, temperatures in a georeferenced, spatiotemporal-sampled, canopy, shaded, $S$. damnosum s.l., riverine, larval habitat possibly as a result of reduced stomatal conductance and latent heat loss (assuming no major shifts in vapor pressure deficent)[4]. Temperature increase may shorten the reproductive phase of determinate vegetated riverine larval habitat canopy cover decreasing the time during which the cover exists and thus the period during which it intercepts light and produces biomass. As such, the responses of leaf and canopy gross photosynthesis in a endmember, geopredictive, autoregressive, canopy, shade-related, $S$. damnosum s.l., riverine, larval habitat, endemic, trasnmissionoriented, risk model may be associated with increasing temperature. Further, $\mathrm{CO}_{2}$ may be readily seasonally quantitated in terms of the temperature and $\mathrm{CO}_{2}$ dependencies based on quantum yield and light-saturated canopy photosynthesis in the endmember, endemic, transmission-oriented, predictive, risk model employing seasonalsampled, georeferenced, canopy-shaded, S. damnosum s.l., riverine, larval habitat, photosynthetic-related, spectral, covariate coefficients in an ArcGIS based non-rectangular hyperbola model. Analytical seasonal expressions for endmember contributions of sun and shade leaves may then lead to instantaneous quantitation of canopy, photosynthesis rates in a robust, seasonal riverine geopredictive, $S$. damnosum s.l., larval habitat, model output.

An ArcGIS generated photosynthetic hyperbolic model for forecasting georeferenced aggregations of prolific canopy, shaded, $S$. damnosum s.l., habitats based on seasonal-sampled, count data for classifying LULC based on endemic transmission zones requires a 
Citation: Jacob B, Novak RJ, Toe L, Sanfo MS, Caliskan S, et al. (2013) Unbiasing a Stochastic Endmember Interpolator Using ENVI Object-Based Classifiers and Boolean Statistics for Forecasting Canopied Simulium damnosum s.I. Larval Habitats in Burkina Faso. J Geophys Remote Sensing 2: 109. doi:10.4172/2169-0049.1000109

number of assumptions. First and foremost, the riverine larval habitat, endmember, canopy, photosynthetic, model analysis would have to be closed, and, the seasonal-sampled, georeferenced, habitat, canopy, surface-related, explanatory, covariate coefficient indicator values would be assumed to be horizontally uniform. Second, an infectious disease epidemiologist or an abatement district manager must assume that an exponential profile of light down the georeferenced, canopy, shade-related, S. damnosum s.l., riverine habitat would utilize the same decay constant for light from different parts of the sky. Instantaneous canopy photosynthesis for spectrally quantitating analytical expressions for sun and shade leaves based on exponential light decay down the canopy and an acclimated non-rectangular hyperbola for leaf photosynthesis assumes decay constant regardless of atmospheric location [5]. Third, the leaf photosynthetic response to incident irradiance in the georeferenced, riverine, larval habitat, model residuals forecasts for efficiently seasonally targeting the canopy, shade-related, endmember, endemic, transmission-oriented, canopy, photosynthetic, covariate coefficients would have to be described by a three-parameter non-rectangular hyperbola (NRH). Finally, the light acclimation at the leaf level in the georefernced, seasonal, sampled, empirical parameter estimator dataset can only occur in one estimator, of the NRH, specifically the one describing the light-saturated photosynthetic rate, which would then be assumed to be proportional to the local, seasonal, averaged canopy, shaded, $S$. damnosum s.l., larval habitat, leaf irradiance values [3].

Further, ArcGIS-based photosynthetic hyperbolic model assumptions have not been extensively researched empirically and

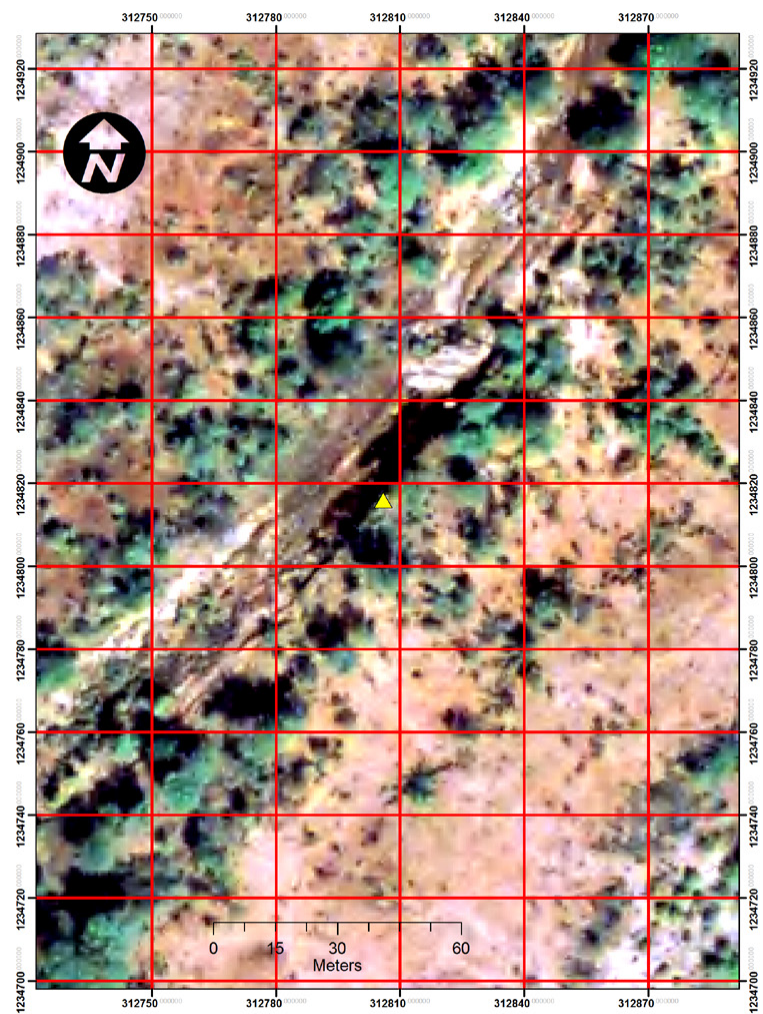

Figure 2: Digitized Grid overlaid onto QuickBird visible and NIR data of the canopy shaded S. damnosum s./ riverine habitat and its land cover attributes.

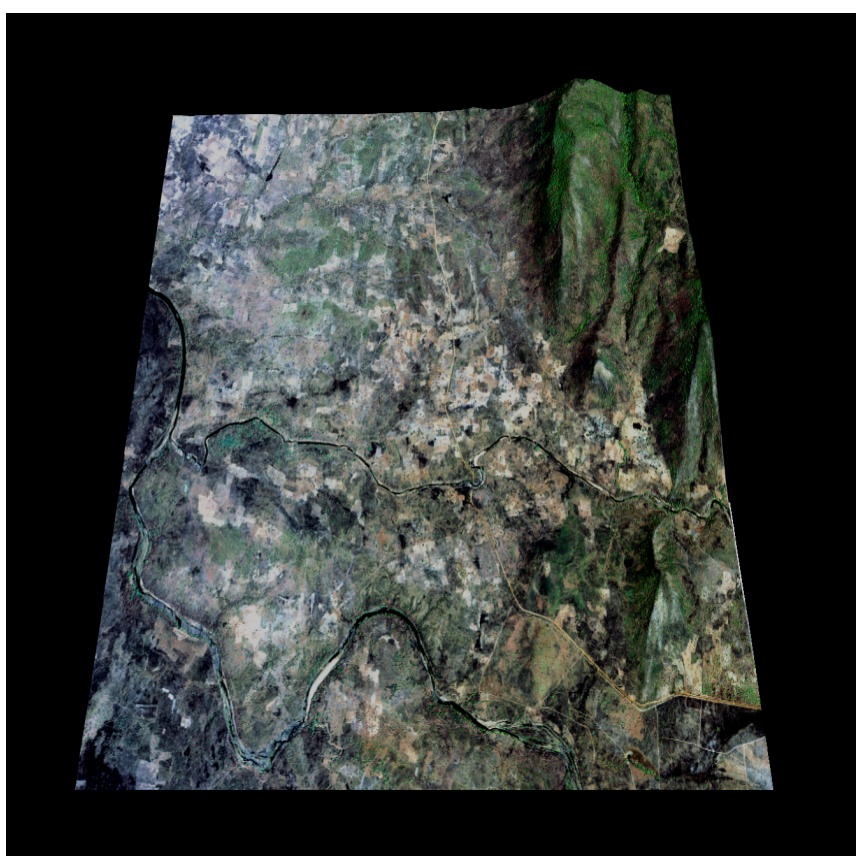

Figure 3: QuickBird digital elevation model of the Dienkoa study site.

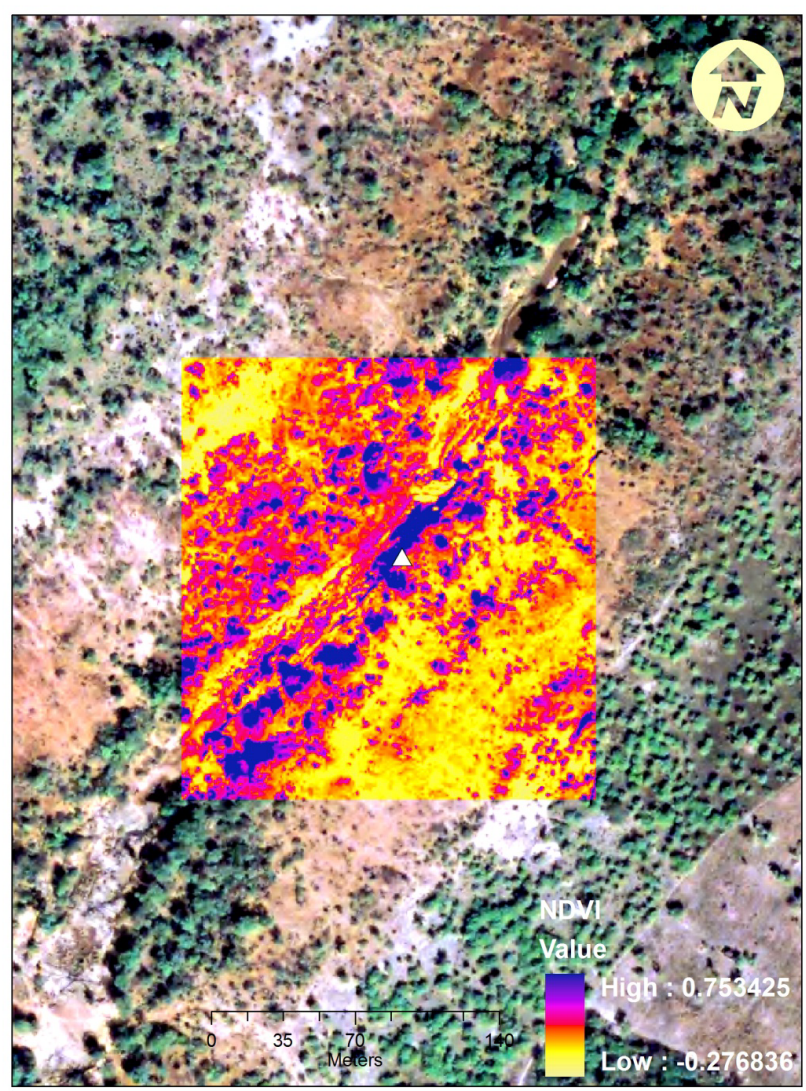

Figure 4: QuickBird NDVI map of the georeferenced canopy shaded S. damnosum $\mathrm{s}$. / habitat at the Dienkoa riverine study site. 

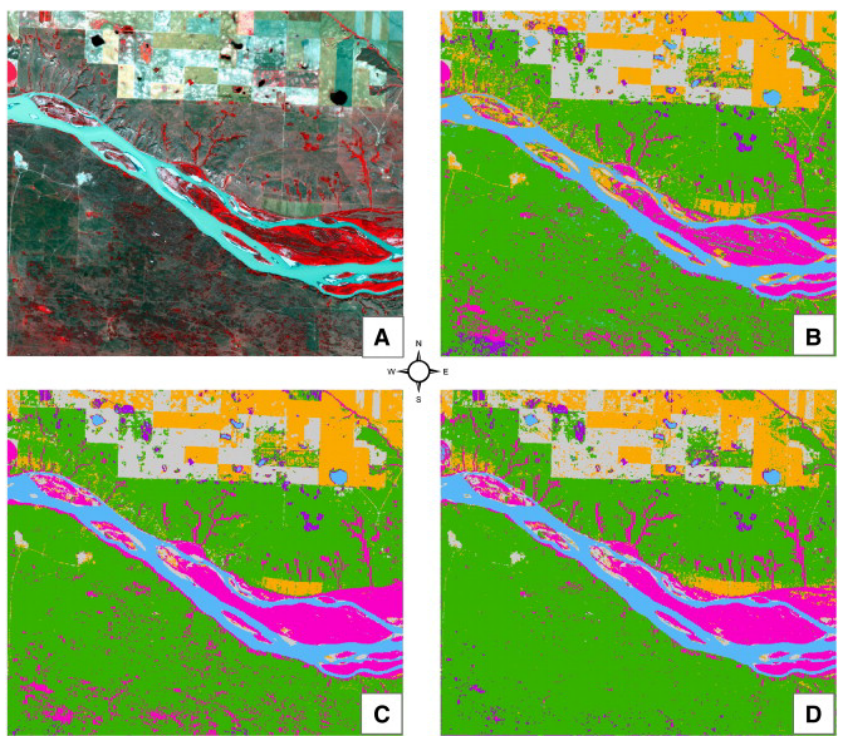

C

\section{Land cover classes}
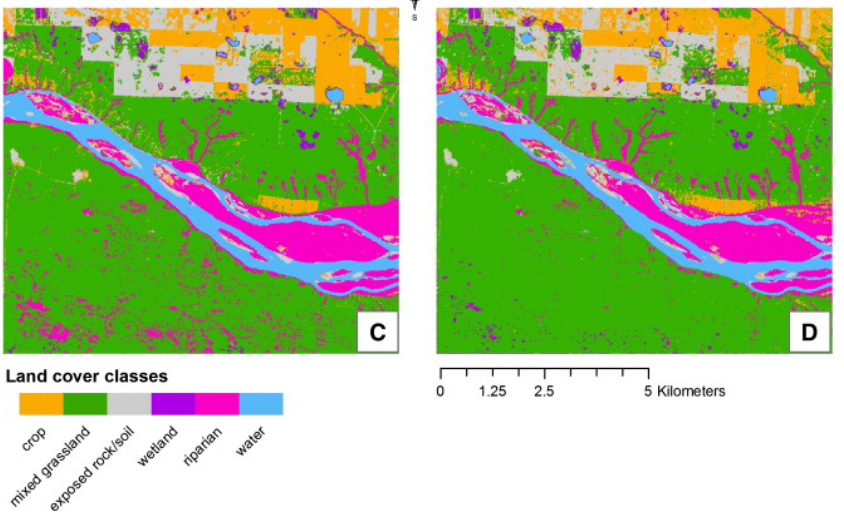

D

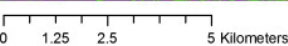

Figure 5: Isodata land cover classificati $\mathrm{n}$ map of the Dienkoa riverine study site.

their limitations are not well understood. As such, there may be other sources of errors embedded in these assumptions that would generate biased residual linear forecasts from a canopy, shade-related, S. damnosum s.l., riverine, larval habitat, geopredictive, endmember, endemic, transmission-oriented, risk model. For example, assuming a horizontal uniform canopy for a georeferenced riverine larval habitat would tend to simplify the different levels of illumination received by canopy leaves in the endemic transmission-oriented model. This simplification, would overestimate the light interception since leaves located in different positions in the canopied, georeferenced, riverine, larval habitat can have different levels of volume and, therefore, illumination. Moreover, assuming an exponential decay of the light within a georefernced, canopied, S. damnosum s.l., riverine habitat would underestimate the amount of light actually intercepted by the leaves located within it. A geopredictive, autoregressive, canopy, shaded, endmember, S. damnosum s.l., riverine, larval habitat, endemic, transmission-oriented, explanatory, risk model based on photon conversion will not seasonally quantitate the stomatal saturation or closing based on the effects of temperature or other abiotic agents, thus overestimating the results in the residual forecasts [5].

In some instances time series canopy radiance uncertainty flux may be described for rapidly computing the amount of solar energy absorbed at the canopy surface of a georeferenced, seasonal-sampled, S. damnosum s.l., riverine, larval habitat as a function of altitude in ArcGIS and ENVI. In previous geopredictive, autoregressive, endemic, transmission-oriented, risk mapping applications, this form of parametric treatment has rendered robust non-arthropod-related empirical datasets of spectrally dependent, georeferenced, explanatory, observational predictors employing accurate multiplescattering quantitation tools. Products in the ENVI platform and IDL deliver off- he shelf and customized image analysis and spectral data visualization solutions which are easily integrated with existing platforms in ArcGIS (www.esri.com). IDL is the trusted scientific programming language used across disciplines to extract meaningful visualizations from complex spectrally-oriented numerical data, providing support for Microsoft Windows ${ }^{\circledR}$, Mac OS X, Linux, and Solaris. (http://www.exelisvis.com/ProductsServices/ IDL.aspx). Thus, IDL/ArcGIS may work seamlessly with ENVI and to provide tools necessary to customize and extend analysis capabilities with ENVI library routines, custom file readers and writers, batch mode programs and user functions for seasonally predicting S. damnosum s.l. riverine larval habitats (Figure 6).

Object-based classification may be a good alternative to the traditional pixel based methods. To overcome problems of segmentation effects, in a robust seasonal, geopredictive, canopy, shade-related, S. damnosum s.l., riverine larval habitat endemic, transmission-oriented risk model, object-based classifiers may analyze groups of contiguous pixels as objects instead of employing the conventional pixel-based classification unit. In previous forest studies, this classification method reduced the local spectral covariate coefficient variation caused by crown textures, gaps and shadows. In addition, with spectrally homogeneous segments of images, both spectral values and spatial properties, such as size, shape and texture can be explicitly utilized as features for further canopy, shade-related, endmember classifications. In theory, this will reduce the local S. damnosum s.l., riverine, larval habitat, canopy, spectral variation caused by crown textures, gaps and shadows [26,15]. In addition, with spectrally homogeneous segments of georeferenced, canopied, riverine, larval habitat images, both spectral values and spatial properties, such as size and shape of the canopy can be explicitly utilized as features for further endmember classification. The basic idea of this process would be to group the spatially adjacent S. damnosum s.l. riverine larval habitat pixels into spectrally homogenous objects first, and then conduct classification on within canopy objects (i.e., Precambrian rocks) employing time series dependent minimum processing units.

Kitting and Land grebe [46] proposed a similar idea and developed the spectral-spatial classifier called extraction and classification of homogeneous objects (ECHO). More recently, some ecological habitat

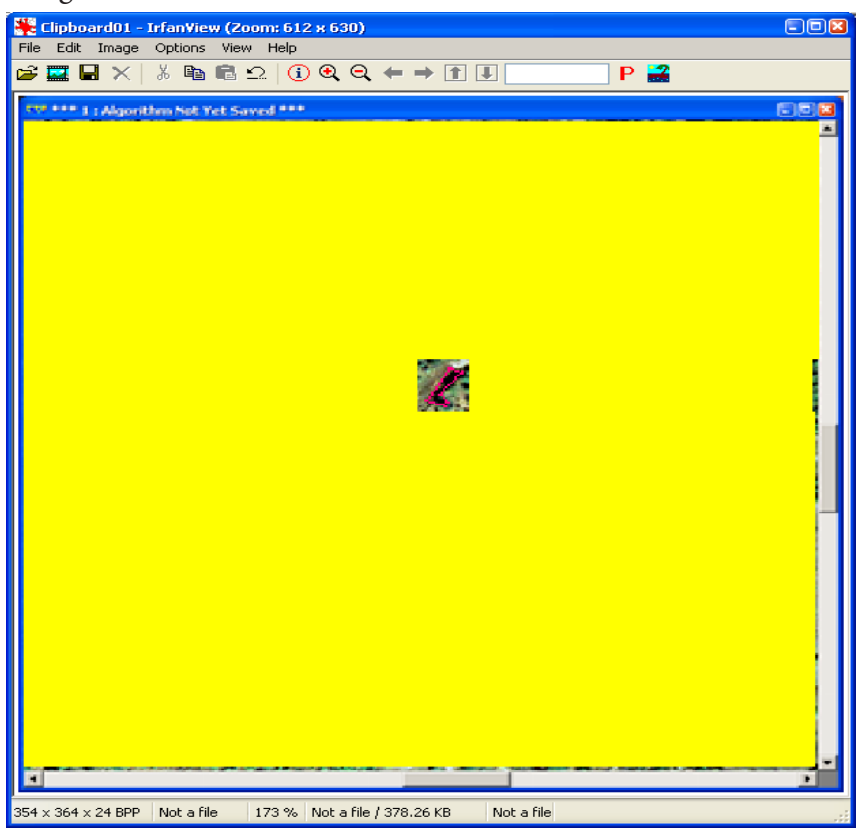

Figure 6: Remote masking of the canopy shaded $S$. damnosum s.l. riverine habitat site and its Precambrian rock and ripple water components. 
autoregressive risk modeling research adopted this method using LULC classifications combined with image interpretation knowledge and spectral classification results were significantly improved [3]. As Kettig and Landgrebe pointed out, the premise of this technique is that the canopied objects of interest (e.g., georeferenced, canopy, shaded, S. damnosum s.l., riverine larval habitats) are proportionally large compared to the size of a pixel. Obviously, this approach was not extensively studied or implemented for LULC mapping at the time when Landsat Thematic Mapper ${ }^{\mathrm{TM}}$ data prevailed as readily available multispectral data for seasonal arthropod-related risk-based analyses for targeting endemic transmission-oriented spatial aggregation sites. An increasing body of research however realizes that the object-based approach would be promising for handling sub-meter resolution imagery (e.g., panchromatic QuickBird data at $0.61 \mathrm{~m}$ resolution).

For a set $\mathrm{P}$ of points (e.g., S. damnosum s.l. larval habiat ground coordinates) in the ( $d$-dimensional) Euclidean space, a Delaunay triangulation is a triangulation $\mathrm{DT}(\mathrm{P})$ such that no point in $\mathrm{P}$ is inside the circum-hypersphere of any simplex in DT(P). The concept of an Euclidean space in a canopy-shaded, $S$. damnosum s.l., related, larval habitat would then encompass Euclidean plane and the 3-D space of Euclidean geometry as spaces of dimensions 2 and 3 respectively. From the modern viewpoint there would be essentially only one Euclidean space for each dimension in the riverine larval habitat model. With Cartesian coordinates this would be modeled by the real coordinate space $\left(\mathrm{R}^{n}\right)$ of the same dimension. In dimension one this is the real line; in dimension two it is the Cartesian plane; and in higher dimensions it is a coordinate space with three or more real number coordinates [1]. It is known that there exists a unique Delaunay triangulation for $\mathrm{P}$, if $\mathrm{P}$ is a set of points in general position [1]. That is, there exists no $\mathrm{k}$-flat containing $k+2$ points nor a $\mathrm{k}$-sphere containing $k+3$ points, for $1 \leq k \leq d-1$ [e.g., for a set of seasonalsampled, canopy, shaded, riverine, larval habitat points in $\mathbb{R}^{3}$; no three points are on a line, no four on a plane, no four are on a circle, and no five on a sphere]. As such, the problem of finding the Delaunay triangulation for a set of seasonal-sampled, canopy, shade-related, riverine, larval habitat points in $d$-dimensional, Euclidean space may be parsimoniously quantitated for finding the convex hull of a set of the sampled larval habitat points in $(d+1)$-dimensional space. This calculation may be performed by calculating each georeferenced point $p$ along with an extra coordinate equal to $|p|_{2}$, taking the bottom side of the convex hull, and mapping back to $d$-dimensional space by deleting the last coordinate. As the convex hull is unique, so is the triangulation, assuming all facets of the convex hull of a georeferenced vector larval habitat are simplexes [2]. Non-simplicial facets only occur when $d+2$ of the original points lie on the same $d$-hypersphere, i.e., the points are not in general position.

Kitting and Landgrebe [46] demonstrated the ECHO method outperformed the conventional textures. However, the authors found that it was not feasible to apply this method to broadleaf forest since treetops could be easily identified. Besides this, few studies have compared the efficiency of an object-based approach with a conventional pixel-based approach for any type of canopied, georeferenced, vector arthropod-related, larval habitat, seasonal, riskbased, endemic, transmission-oriented, geopredictive explanatory data analyses. Thus, studies on detailed seasonal vegetation mapping with widely-used high-resolution multispectral imagery and object-based classifiers would be worthwhile for S. damnosum s.l. riverine larval habitat canopy endmember risk modeling even though there would be some difficulties based on meteorological parameters such as type of clouds, the humidity, the zenith angle of the sun, the albedo of the earth's surface and others which would influence canopy, shaded, diffuse scattering of the georeferenced larval habitat [2]. Effects on stratosphere-oriented observations and canopy absorption of georeferenced, canopied, S. damnosum s.l., riverine, larval habitats may be parsimoniously, spectrally quantitated and spatially summarized employing object-based classifiers and seasonal-based calculations of the vertical distribution of the ozone.

Presently published literature provides an extensive list of different parameterization schemes for unmixing, georeferenced, canopy, shaded, S. damnosum s.l., riverine, larval habitat mixes for quantitating cartographic data seasonally delineating canopied, spectral, endmember reflectance properties. For example, parameterization closure schemes in numerical, seasonal, meteorologically-oriented, endmember, $S$. damnosum s.l., riverine, larval habitat, endemic, transmission-oriented, risk models can account for the effects of canopied physical processes. Unfortunately, they cannot be resolved explicitly by standard unfixing models. These models would include: Pixel Purity Index N finder algorithm, Iterative Error Analysis, Vertex Component Analyses, Optical Real-time Adaptive Spectral Identification System (ORASIS), Segmentation Maximum Angle Convex Cone, Iterated Constrained Endmember, Minimum Volume Constrained Non-negative Factorization and Simplex Growing Algorithm. Methods for determining robust physical canopy-oriented riverine larval habitat parameterization schemes may require introducing differential equations after the endmember unmixing scheme.

Frequently graphical and numerical methods have been applied to generate solutions in geopredictive, regression-based equations [2,47]. Additionally, since remote detection methods for risk mapping $S$. damnosum s.l. endemic transmission zones based on spatial aggregations of productive habitats rest on the possibility of spectrally delineating and interpolating derivatives from a canopy-oriented endmember parameterization scheme, employing various classes of differential equations would be beneficial. For instance, relevant canopy endmember classification problems may be solved using direct or inverse spectral endmember classification procedures. Thereafter, if an infectious disease epidemiologist or a local abatement district manager employs the direct approach, he or she could generate a general functional form of the parameterization scheme for spectrally unmixing a dataset of sub-meter resolution spatiotemporal-sampled, georeferenced, canopy, shaded, endmember, S. damnosum s.l., riverine, larval habitat, geopredictive, endemic, transmission-oriented, autoregressive, risk model parameter estimators. The characteristic property of the spatiotemporal, regression-based forecasting equations would then be based on their solutions forming an affine subspace of an appropriate, canopied, endmember, function space, which in turn would result in many optimal linear differential equations being generated in ArcGIS. For example, homogeneous linear differential geopredictive equations (e.g., Ordinary kriged model) could be constructed once a affine subspace is defined in a time-series dependent, canopied, riverine, larval habitat, endmember, geopredictive, autoregressive, risk-based model for efficiently targeting, endemic, transmission, oriented, endmember, photosynthetic and non-photosynthetic explanatory covariate coefficients. This model would be a subclass for which the space of solutions in a spatiotemporal, explanatory risk, riverine larval habitat geopredictive endemic transmission-oriented, model could be employed for quantitatively spectrally analyzing the fractional canopyoriented photosynthetic and non-photosynthetic explanatory endmember covariate coefficients. The residuals would actually then be spectrally seasonally quantitated employing a linear subspace in the sampled parameter estimation regression-based matrix framework, whereby the sum of any set of solutions or multiples of solutions would also be a solution in the georeferenced riverine larval habitat 
endmember endemic transmission-oriented risk model. However, the coefficients of the unknown function (i.e., dependent variable) and its derivatives in a seasonal, canopy, shaded, S. damnosum s.l., riverine, endmember, larval habitat, geopredictive, differential regression-based equation would not be allowed to be functions of the independent variable in the model residual forecast estimates.

Further, special attention can be paid to the problem of finding parameterization schemes in interpolatable residually forecasted estimates that preserve geometrical, canopied, seasonal-sampled, georeferenced, S. damnosum s.l., riverine, larval habitat, optical symmetries. These methods may find robust solutions to conservative invariant parameterization schemes for systems seasonally representing a one-dimensional canopied, S. damnosum s.l., larval habitat, endmember-related, geopredictive, regression-based interpolation equation [3]. Specific forms can be rendered so that corresponding closed equations can then conform to unmixing ArcGIS-related and ENVI residual spectral classifications [e.g., Spectral Angle Mapper (SAM) and Spectral Information Divergence (SID) Classifications]. Spectral Angle Mapper is a deterministic method that looks for an exact pixel match and weights the differences the same while SID Classification is a probabilistic method that allows for variations in pixel measurements, where probability is measured from zero to a user-defined threshold (www.exelisvis.com).

A differential inverse approach and object-based classifiers can be employed for time series explanatory endmember $S$. damnosum s.l. riverine larval habitat modeling, whereby parameterization schemes preserve one or more pre-selected spectrally unmixed canopied endmember photosynthetic and non photosynthetic explanatory covariate coefficients regressed in the initial ArcGIS/ENVI-related model. However, these schemes would be based on many assumptions which may fail or give an inadequate response to certain synoptic forces limiting their application in a time series-dependent georeferenced canopy shaded S. damnosum s.l. riverine larval habitat seasonal endmember endemic transmission-oriented geopredictive explanatory risk model. For example, in order to achieve realistic interpolated model simulations of seasonal spatially aggregated, georeferenced, prolific, habitats, the governing equations must deal adequately with nonlinear partial differential riverine, larval, habitat, residual, canopied, endmember regression-based uncertainties. These unqunatiated residual error prone covariates would have to be discretized. A finite grid resolution would have to be chosen to determine which sampled ecological processes cannot be resolved by the resulting endemic transmission-oriented model residual forecasts. This would lead directly back to the parameterization problem in the initial georeferenced risk-related endmember explanatory riverine, larval habitat model since the processes of the sub-grid scale of the geopredictive, canopy, shaded, endmember model would have to be somehow included in terms of the grid scale quantities.

Alternatively, it may be illustrated how the georeferenced, canopied, riverin, larval habitat symmetries can be used to provide a generalized regression-based framework for various classes of parameterization schemes with prescribed geometric properties. The question would arise only based on how these georeferenced riverine larval habitat symmetries are linked to the parameterization processes in the unmixing model. Parameterizations for the unknown quantities should be physically reasonable, have the same dimensions as the unknown, have the same tensor properties, be invariant under an arbitrary transformation of coordinates systems and be invariant under Galilean transformation (i.e., inertial, Newton) [1]. Further, parameterization schemes commonly apply several assumptions and approximations to simplify spectrally-oriented unresolved residual algorithmic processes which typically include moist convection, atmospheric turbulence, radiative transfer, microphysics, soil and vegetation interaction [1]. Unfortunately, these assumptions would cause spectral bias in seasonal, canopy, shade-related, photosynthetic, endmember, $S$. damnosum s.l., riverine, larval habitat, reflectance estimates (i.e., incorrect SSI values) thus, rendering misspecified biased linear estimators within interpolated explanatory time series geopredictive regression-based framework.

The proven strengths of non-parametric spectral analysis may lead to improved robust, seasonal, canopied, vector arthropod-related, infectious disease, geopredictive, endmember, larval habitat models for risk mapping endemic transmission zones. Broadly speaking, spectral unmixing is a special case of the generalized inverse problem that seasonally endures when estimating sampled density count values and their tabulated prevalence rates and canopy system endmember parameters using one or more observations of a sensor signal. In the case of hyperspectral sensing in the reflective regime, the incident signal is EM originating from the sun, which is measured by a sensor after the radiation, has been reflected upward by natural and manmade materials on the surface of the earth [5]. These strengths may lie in three major areas for robust, seasonal, canopied, S. damnosum s.l., riverine, larval habitat, geopredictive, time series, explanatory epidemiological, risk modeling. First, a non-parametric spectral analysis is a physically based model that can transform canopy riverine larval habitat radiance or reflectance values to physical, exploratory, predictor variables where the residual forecasts may be linked to the sub-pixel abundances of canopy endmembers within each pixel. Second, a non-parametric spectral analysis may provide a means to detect and represent within-canopy, shaded, spatiotemporal components that occur entirely at a sub-pixel level, such as sparse floating vegetation in an arid riverine environment. S. damnosum s.l. are also known to breed in low lying riverbeds where they are attached to rocks and also Pennisetum (e.g., horse tails) and to floating masses of Echinochloa. Additionally, the adults rest on vegetation on the islands in the rapids with the male clustering on the flowers of Baphia [47]. Finally, a robust, non-parametric model could provide quantitative results which could be incorporated into models employing the processes governing the distribution of georeferenced materials (e.g., vegetation and turbidity-related fractional radiance endmembers) within an image pixel. Thus, spatiotemporal, geopredictive, endmember, risk mapping, georeferenced, varying and constant, spectral, explanatory, predictor covariates rendered from an spectral unmixing algorithm may then reveal unsampled habitats associated to turbidity and vegetation-oriented, endemic, transmission-oriented, parameter estimators, for instance, within an interpolator while subsequently delineating essential requirements for the development of $S$. damnosum s.l. pre-imaginal stages.

Variations in the abundance of floating vegetation within a seasonal, canopy, shaded, S. damnosum s.l., habitat scene can be directly entered into ArcGIS cyberenvironment for geopredictively risk mapping endemic transmission zones based on aggregations of seasonally prolific habitats as classified by field-sampled, larval, density, count data. The underlying assumption of the nonparametric model, given linear mixing systematics, would be that each spectrally extracted riverine larval habitat sub- meter resolution, subpixel photosynthetic explanatory covariate coefficients on the canopy surface would be a physical mixture of multiple components weighted by canopy surface abundance. As such, the spectrum of the canopied larval habitat mixture can be seasonally quantitated thereafter employing a linear combination of the canopy, endmember, fractional reflectance spectra. By so doing, the spectral variability of the georeferenced, canopy, shaded, S. damnosum s.l., riverine, habitat scene can then be modeled as a linear combination of a small number 
of extracted spectral endmembers whose abundance within each pixel can be seasonally calculated. Theoretically, an empirical dataset of canopy, shaded, non-parametric-derived S. damnosum s.l. larval habitat endmembers could be then selected from a image cube C image endmember that best accounts for the cube's spectral variance within a constrained least-squares mixing model. Ideally, these model residual forecasts can be compared to "reference endmembers", which would essentially entail quantitating the field or laboratory sampled spectra, for calibration and interpretation of the seasonal imaged riverine larval habitat data. The abundance of the canopy, shaded, endmembers within the image would then be by "fraction images". These images would then be employed to investigate physical processes that are related to the $S$. damnosum s.l., riverine, larval habitat, canopy, shade-related, surface abundance, using varying ecosystem models and LULC change detection studies. The canopied endmember fractional riverine, larval habitat, residual, spectral, photosynthetic, explanatory covariate coefficients would then be able to efficiently render unbiased linear estimators in a quantitative deterministic/stochastic-oriented, geopredictive, explanatory, time series, autoregressive,endemic, transmission-oriented, risk-based model.

Accuracy of vegetation and other LULC fractions, computed with just a non-parametric model, may be misspecified by variation in canopy structure and biochemistry especially when quantitating a single canopied endmember spectrum representing the TOC reflectance of a georeferenced, S. damnosum s.l., riverine, endmember, larval habitat. Applied to sub-meter resolution remotely sensed images, for example, improper bundle unmixing can produce misspecified maximum and minimum images by rendering incorrect, canopied, cover, fractionalized values while simultaneously propagating the spectral error due to initial spectral non-quantitation of the canopy-oriented, photosynthetic, endmember variabilities [5]. While these results would be repeatable for a given set of time series, dependent, S. damnosum s.l., riverine, larval habitat, canopy, endmembers, if the endmembers are incorrect in a physical sense, then the fractional abundances would also be incorrect and therefore the interpolated stochastically/deterministically, estimates targeting the endemic, transmission-oriented, explanatory, time-series covariate, Currently, coefficient canopy. endmembers for pixel decomposition are selected through an educated tiral-and-error process employing the Principals Components Analyses [PCA] and convex hull geometrical algorithms [2]. This method has been

considered an innovative approach for canopied aquatic larval habitat endmember, time series, geopredictive, endemic, transmissionoriented, risk modeling since endmember selection is fitted using an $n$-dimensional polyhedron to the data cloud. Past studies of residual uncertainty handling with polyhedral clouds have already shown strength in dealing with higher dimensional uncertainties in robust canopied, endmember, fractional, radiance, optimization procedures [3]. In this technique, a statistical data transformation similar to PCA would be seasonally employed to spectrally quantitate the number of canopy, shaded, S. damnosum s.l., riverine, larval habitat, endemic, transmission-oriented, canopy, endmembers in an empirical sampled dataset which would then define the dimensionality of the habitat polyhedron. The edges and planes of the data cloud (e.g., two- and three-component mixing lines) would then guide the fitting process in ArcGIS. Thereafter, the vertices of the polyhedron would indicate the spectrally significant canopied spectral endmembers related to the georeferenced riverine larval habitat. This method would require no prior knowledge of the image scene or spectral properties of the georeferenced riverine larval habitat within canopied materials in the scene.
One difficulty, with such an approach by itself for seasonal, geopredictive, canopy, shaded, S. damnosum s.l., larval habitat endmember endemic, transmission oriented, risk modeling is that data clouds without straight lines along the edges may not fit uniquely. Furthermore, the calculated S. damnosum s.l., related endmember canopy spectra may not be realistic in a physical sense. Although the residual and RSME of the canopy, endmember, larval habitat, geopredictive, autoregressive, endemic, transmission-oriented risk model would reveal spatially coherent areas of negative or super positive spectral fractions which could serve to improve the initial, canopied, riverine, larval habitat, endmember selection, it would be a very difficult task to determine a good mathematical description for residual uncertainty within such a framework. Among the most critical issue in endmember, time series, dependent modeling would be lack of statistical information and the well-known curse of dimensionality in the residual, estimated, canopy-oriented, linear predictors [5].

Canopy 3-dimensional (3-D) radioactive transfer models simulate transfer processes in the solar domain at or near the Earth's terrestrial surface, (i.e., within plant canopies and over bare soil surfaces) [1]. Such models may be capable of simulating the transmitted, reflected and absorbed radiation fluxes, as well as, the angular distribution of the reflected light above a canopy, shaded, $S$. damnosum s.l., riverine, larval habitat surface in the optical domain of the solar spectrum. Several models exist for the calculation of 3-D canopy radiative transfer although 3-D canopy-oriented radiative transfer models may be optimally linked to atmospheric radiative transfer models by the offline simulation, which would describe the angular distribution of atmosphere or canopy radiation at the atmosphere-canopy boundary via parametric interfaces [e.g. 3]. As such, the most convenient approach for quantitatively investigating the relationship between atmospheric properties and the 3-D canopy light environment in a riverine, larval habitat, endmember, reflectance model would be to employ a fully coupled, atmosphere-canopied 3-D, radiative transfer spectral analyses for accurate treatment of the multiple within and external, canopy, endmember, diffuse radiance scattering feature data attributes. Directional aspects are important for classifying spectrally extracted endmember weights since the brightness with which a given canopy target appears in Earth Observation data. This depends not only on the structural and spectral properties of the target but also on the viewing and illumination geometry at the time of measurement [1].

Laboratory measurements rendered from a 3-D radiative-transferbased canopy, oriented, riverine, larval habitat, plane-parallel models can be constructed in an ArcGIS geodatabase for spectrally quantitating bidirectional reflectance distribution function (BRDF). The BRDF is a four-dimensional function that defines how light is reflected at an opaque surface [4]. This function would be dependent on specific wavelength combinations (i.e., scattergram), $S$. damnosum s.l., larval habitat structural and optical properties of the canopy surface (e.g., reflection, absorption, shadow-casting, multiple scattering mutual shadowing of the canopy surface elements, facet orientation distribution and facet density). The ratio of the radiance directional fluxes traveling upward and downward through an infinitesimal area (i.e., the bidirectional reflectance), can act as the product of the BRDF at a particular geographic sampled point and the projected direction of reflection.

Previous approaches using BRDF have included two-stream, radiative transfer, geometric optics, hybrids of two or more of these and numerical simulation. For example, Li and Strahler [51] emphasized the individual tree canopy as the functional element in numerous canopy endmember reflectance models by 
applying geometric-optical algorithms for spectrally quantitating bidirectional reflectance successfully in open and moderately closed stands of conifers treated as "green" cones. Jupp et al. [52] used a similar approach for trees as spheroidal objects, and extended the treatment to two crown layers above a background using Boolean logic. Further, Strahler and Jupp [52] provided a general Boolean treatment that included leaves within discrete crown envelopes as a two-stage nested model. Canopy biophysical parameter retrieval via BRDF inversion over heterogeneous regions is highly affected by the spatial resolution of the angular reflectance data [2]. An unmixing methodology may be presented which utilizes sub-meter resolution imagery, and a geometrical-optical model inversion to recover reflectance of individual, within-canopied, landcover types in riverine-based regions.

In this research, the BRDF function was calculated based on the geometrical shape of canopy, shaded, spatiotemporal-sampled, $S$. damnosum s.l., larval habitat components, their density, their brightness relative to the thickness of the canopy layer and shadowed data feature attributes surrounding a georeferenced habitat (e.g., Precambrian-rock, floating leaves) sampled in Dienkoa riverine village study site in Burkina Faso. We spectrally, decomposed, a submeter spatial resolution (i.e., QuickBird seasonal-sampled $S$. damnosum s.l. larval habitat pixel using an array of unmixing algorithms, object-based classifiers and, then, calculated the BRDF function within a Boolean domain for geographically predicting productive larval habitats within the riverine ecosystem study site based on a spectral signature and quantitated spatiotemporal fieldsampled count data. Initially, individual sub-pixel spectral reflectance estimates from a QuickBird visible and near-infra-red (NIR) at $0.61 \mathrm{~m}$ spatial resolution data of a georeferenced habitat (i.e., capture point) was extracted by using a radiative transfer model and the Li-Strahler geometric-optical model. The model employed three scene components: Precambrian Rock in sunlit canopy (P), white sunlit turbid-related data $(\mathrm{T})$ and vegetation-oriented shadow background $(\mathrm{V})$, generated from the image. The $\mathrm{P}, \mathrm{T}, \mathrm{V}$, components' classes were then estimated using ENVI, an object-based classification algorithm. In $\mathrm{ENVI}^{\circledR}$, the Digital Number (DN) of the pixel in every QuickBird

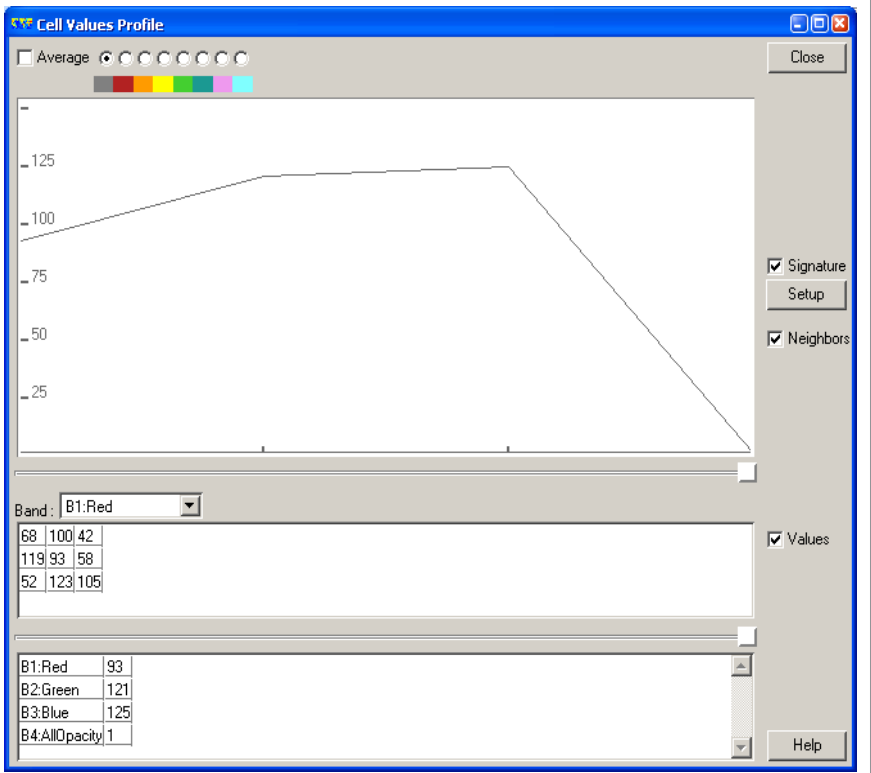

Figure 7: Quantifying S.damnosum s.l. habitat pixel endmember spectra including the Precambrian rock and ripple water pixel spectra from a QuickBird spectrally decomposed pixel. band was viewed using the $z$-profile from a spectral library. After making an atmospheric correction from the image for the study site, the DN was then converted into ground reflectance (Figure 7).

During our model construction phase, we generated multiple riverine LULC datasets of remotely synthesized, geomorphological, terrain-related, explanatory covariate coefficients from digital surfacebased, canopy, shaded, geopredictive, S. damnosum s.l., larval habitat models using the QuickBird visible and NIR data. Digital surface models are topographic maps that provide a geometrically correct reference frame over which other satellite data layers can be draped [3]. A digitized grid-based matrix was then overlaid onto the georeferenced riverine habitat in ArcGIS for managing the spatiotemporal-sampled parameter estimators and for generating an efficient mathematicalbased sample frame. For remote identification of georeferenced arthropod-related infectious disease immature habitats, the first step is often to construct a discrete tessellation of the region [9]. Normalized Difference Vegetation Index (NDVI) parameters were then generated from the QuickBird data. Sub-meter spatial resolution reflectance is affected by BRDF and, thus, vegetation indices (VI) and nadir-normalized reflectance for identifying vector arthropod-related larval habitat suitability [10]. We also constructed a robust DEM for identifying important geomorphologic-based, terrain-related, explanatory, predictor, covariate coefficient estimates associated to the seasonal-sampled, canopy, shaded, S. damnosums s.l., riverine, habitats using the QuickBird-derived LULC data.

Once the LULC related predictors were determined, we employed ENVI $^{\circledR}$ technology and a Successive Progressive Algorithm (SPA) algorithm to extract and unmix the canopy-dependent emissivities from within the QuickBird S. damnosum s.l. riverine habitat pixel spectrum. We encompassed the effects of the remotely captured riverine topographical data using the $\mathrm{BRDF}$ and the hemispherical reflectance (i.e., surface albedo) of the Precambrian rock and also of the decomposed, rippled water, canopy, shade-related, photosynthetic, spectral, explanatory, endmember components associated to the georeferenced, canopied, S. damnosum s.l., riverine, larval habitat in the geometric-optical model. A convex geometrical model was then used for canopy endmember validation derived from the spectrally decomposed riverine habitat data. We then employed an Ordinary kriged-based interpolator to forecast other prolific habitats based on the $S$. damnosum s.l. riverine habitat canopy radiance estimates and its associated spectral endmember constituents. Ordinary kriging is a geostatistical approach which relies on the spatial structure of the data to determine the weighting values, including rigorous modeling of correlation between data points for determining the estimated value at an unsampled point [13].The ordinary kriged-based interpolation was performed in $\operatorname{ArcGIS}^{\circledR}$ Geostatistical Analyst using the target signature generated from the unmixing models. Linear unbiased, explanatory, predictors and variance estimates were then derived for all the productive, canopy, shaded, S. damnosum s.l., larval habitats in the study site based on the extracted pixel endmember reflectance estimates.

The desire to extract from a time series, dependent, canopy, shaded, S. damnosum s.l. riverine, larval habitat, geopredictive endmember spectrum the constituent materials in the mixture, as well as the proportions in which they appear may be important to numerous control oriented cost-effective tactical scenarios in which sub-pixel detail would be considered valuable. Further, unlike previous tactics for unmixing canopied objects which relied on a single unmixing technique and coarse resolution data, in this research we employed multiple algorithms and remote sensing models to extract a robust riverine larval habitat 
Citation: Jacob B, Novak RJ, Toe L, Sanfo MS, Caliskan S, et al. (2013) Unbiasing a Stochastic Endmember Interpolator Using ENVI Object-Based Classifiers and Boolean Statistics for Forecasting Canopied Simulium damnosum s.l. Larval Habitats in Burkina Faso. J Geophys Remote Sensing 2: 109. doi:10.4172/2169-0049.1000109

Page 17 of 36

sub-meter resolution target signature. Our assumption was that by using multiple unmixing algorithms, sub-meter resolution imagery, remote sensing models and object based classifiers (e.g., ENVI technology), we would be able to seasonally spectrally quantitate all $S$. damnosum s.l. riverine larval habitat canopy, shade-related radiance. Therefore, our research objectives were to: a) remotely synthesize NDVI and DEM-related, georeferenced, geomorphological, explanatory predictor covariate coefficient estimates associated to the various epidemiological riverine larval habitat study site LULC areas; b) conduct a sub-pixel decomposition for digitally segmenting the endmembers based on spatial, spectral and texture characteristics using an $\mathrm{ENVI}^{\circ}$-oriented object-based classifiers; c) establish the sensitivity and dynamic ranges of a 3-D canopy-oriented radiative transfer model employing SPA algorithm and a robust geometricoptical model for quantitating canopy, shaded, endmember, fractional abundances values (i.e., geosampled habitat's Precambrian rock with rippled water reflectance values); and, d) construct and validate spatial linear-based explanatory predictors from an interpolation-based algorithm using the spectra canopy, shaded, endmembers decomposed from a QuickBird $0.61 \mathrm{~m}$ pixel for spatially targeting aggregation of productive S. damnosum s.l. habitats based on spatiotemporal, field-sampled count data (Figure 8).

\section{Material and Methodology}

\section{Study site}

This research was conducted at Dienkoa riverine village site in Burkina Faso, a landlocked country in West Africa. The study site has three distinct seasons: warm and dry (November-March), hot and dry (March-May), and hot and wet (June-October). Annual rainfall varies from about $250 \mathrm{~mm}$ to $1,000 \mathrm{~mm}$. The terrain at the riverine site is mostly flat with undulating plains and hills. Most of the study site region lies on a savanna plateau, with fields, brush, and scattered trees. The geological history of the site is marked by Precambrian volcanic activity and Eburnean faulting and folding. These geological events, followed by successive erosion cycles have given rise to the basis of the relief in some of the study site which are comprised of peneplain and sandstone plateaux. There are seven main soil types in the river ecosystems which include: leached ferruginous, brown eutrophic, vertisols, ferralitic, halomorphic, hydromorphic and raw mineral soils. The first two soils cover more than two thirds of the riverine study site. In the study site, there are also four types of pasture vegetation which include: 1) savanna with sparse tree cover on

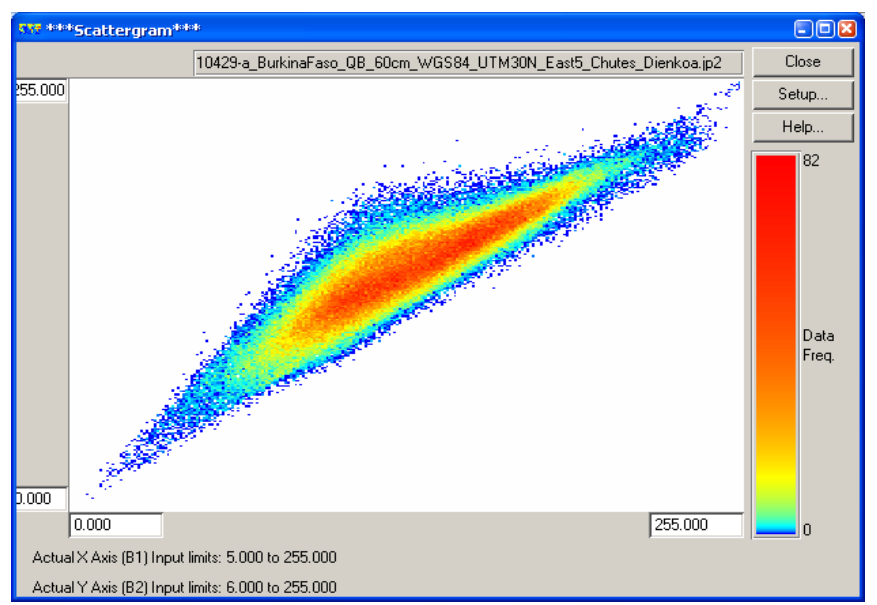

Figure 8: An endmember reference signature of a canopy shaded S.damnosum s.l. habitat and its Precambrian rock and ripple water pixel spectral re lectance values.

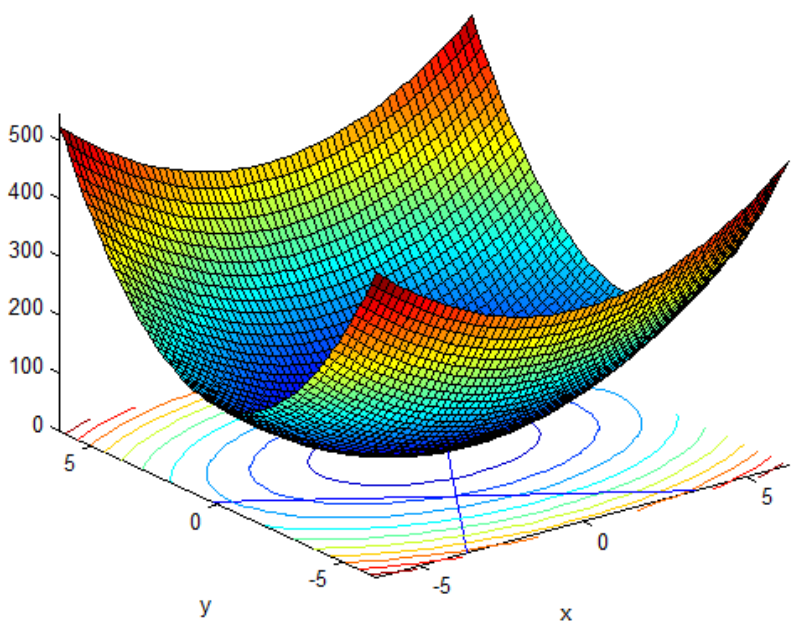

Figure 9: Quadric surface of the canopy shaded S. damnosum s.l. riverine habitat at the Dienkoa study site area expressed as a hyperbolic paraboloid with contour line and constraints.

buttes with laterite pans including such plants as Butyrospermum paradoxum, Burkea africana, Loudetiopsis scaëttae and Schizachyrium sanguineum; 2) treed savannas with Isoberlinia doka and Andropogon ascinodis; and, 3) grassy savanna on bowal with Loudetia togoensis and Diheteropogon hagerupii; savannas in temporarily flooded riverine areas with Imperata cylindrica and Schizachyrium brevifolium; and, grasslands of land flooded for long periods with Oryza barthii and Acroceras amplectens. The basin of the Niger River drains $27 \%$ of the Dienkoa study site surface area. The Niger's tributaries - the Béli, the Gorouol, the Goudébo and the Dargol - are seasonal streams and flow for only four to six months a year (Figure 5, Figure 9).

\section{Remote sensing data}

A stereo image of QuickBird was obtained from the DigitalGlobe on the 15 July 2010, for a $64 \mathrm{~km}$ region of the epidemiological study site area. The imagery was classified using the Iterative Self-Organizing Data Analysis Technique (ISODATA) unsupervised routine in ERDAS Imaginev.8.7 $7^{\mathrm{TM}}$ (ERDAS, Inc., Atlanta, Georgia). Unsupervised classifications are commonly used for the identification of sub-meter resolutionderived LULC classes associated with prolific vector arthropodrelated habitats based on spatiotemporal-field-sampled count data [1,46]. In previous research, Krishana et al. [14] used QuickBird visible and NIR data for imaging shallow ground water wells in the Atankwidi Watershed in the Volta River basin. The QuickBird sensors were able to identify dug wells that were 1 to 2 $\mathrm{cm}$ in depth. Results revealed that well-digging was practiced on 387 (1.4\%) rainfed land cover areas, 15,638 (54.7\%) in dryer arid regions and the remainder in other ArcGIS-classified LULC areas. The field-plot revealed an accuracy of $92 \%$ with an error of omission and commission of less than $10 \%$.

\section{Habitat mapping}

Base maps were generated using the QuickBird visible and NIR data and differentially corrected global positioning systems (DGPS) ground coordinates of 31 sampled S. damnsoum s.l. riverine larval habitats. The differentially-based ground coordinates were acquired from a CSI max receiver which has a positional accuracy of \pm .178 . Using a local DGPS broadcaster can compensate for ionospheric and 
ephemer its effects, which can improve horizontal accuracy significantly and can bring altitude error down in a predictive vector arthropod aquatic larval habitat endmember endemic transmissionoriented model [1]. Each georeferenced, canopy, shaded, $S$. damnosum s.l., larval habitat was entered into the $\mathrm{VCMS}^{\mathrm{TM}}$ relational database software product (Clarke Mosquito Control Products, Roselle, IL). VCMS, including connectivity with hand held computers, and field data collection devices, including DGPS receivers, Palm OS and Windows Pocket PC hand helds have been used for malaria [12]West Nile Virus (WNV) [13], Eastern Equine Encephalitis Virus (EEEV) [14] mosquito-related larval habitat monitoring. Additional geocoding and spatial display of the spatiotemporal-sampled ecological data was handled using the embedded VCMS ${ }^{\mathrm{TM}}$ GIS Interface $\mathrm{Kit}^{\mathrm{TM}}$, which in this research was developed utilizing ESRI's Map Objects ${ }^{\mathrm{TM}} 2$ technology. The $\mathrm{VCMS}^{\mathrm{TM}}$ database supported the export of all field data using any combination of the spatiotemporalsampled georeferenced $S$. damnosum s.l. habitat parameter estimates in order to further process and spatially display specific data attributes in a stand-alone desktop GIS software package (i.e., ArcGIS $10.1^{\circledR}$ ) (Figure 2).

A digitized grid-based algorithm was constructed in ArcGIS by applying a mathematical algorithm in order to fit the continuous and bounded sampled larval habitat surfaces from a field-sampled attribute. In this research, attributes for an integer grid representing the discrete data, were stored in a Value Attribute Table (VAT). The VAT had one record for each unique sampled, georeferenced, canopy, shaded, S. damnosum s.l., riverine, larval habitat attribute value in the grid. GIS grid-based data files consist of columns and rows of uniform cells coded according to data values [1]. The grid was implemented using a tiled raster data structure in which the basic unit of data storage in ArcGIS was a rectangular block of cells. Blocks are stored on disks in compressed form in a variable-length file structure referred to as a tile (www.erri.com). In this research, each block was stored as one variable-length record.

Multiple data layers were created using different coded values for various field attributes which were related to the same grid cell. Programs that manipulate grids access the spatial data by setting a rectangular window defined in map coordinates (www.esri.com). Each habitat grid cell/polygon was assigned a unique identifier. Field attribute tables were then linked to the polygons. The polygons were used to define the sampling frame, which extended to include a $5 \mathrm{~km}$ buffer from the external boundary of the spatiotemporal-sampled, georeferenced, canopy, shaded, S. damnosum s.l., habitat site. This allowed for multiple interactions enabling retrieval and transformation of the within sampled data feature attributes (e.g., Precambrian rock) efficiently, regardless of spatial dimensionality of the habitat.

\section{Environmental parameters}

Multiple georeferenced predictors were then examined extensively using georeferenced and altitude data. The criteria involved the centrographic measures of spatial mean and distance between a sampled, canopy, shaded, S. damnosum s.l., riverine, larval habitat (i.e., capture point) and the habitat distance from the site to the nearest human habitation village sample and to other habitats. The data was also comprised of individual spatiotemporal-sampled observations of the georeferenced riverine larval habitats together with a battery of categorical attribute measures which were expanded into multiple spectrally-dependent, explanatory, predictor, covariate, coefficient estimates. The data was then analyzed for statistical correlations using Monthly Biting Rates (MBR) and the distance from the georeferenced riverine larval habitats to surrounding villages.
The MBR is commonly calculated by dividing the number of flies caught by the number of catching days and then multiplying the daily biting rate by the number of days in the month [15].

The habitat distances were then measured as Euclidean distances in the projection units of the raster which were computed within the digitized grid cell matrix. The Euclidean distance output raster contained the measured distances. The Euclidean Distance functions provided information according to Euclidean or, straight-line, distance between the georeferenced habitats and from habitats to human habitation (i.e., geometric distances in the multidimensional space) (www.esri.com). In this research the Euclidean distances were computed as: distance $(\mathrm{x}, \mathrm{y})=\left\{\sum_{i}\left(x_{i}-y_{i}\right)^{2}\right\}^{1 / 2}$. Every cell in the Euclidean allocation output raster was assigned the value of the source to which it was closest. The nearest source was determined by the Euclidean Distance function in ArcGIS. This function assigned space between the georeferenced, canopy, shaded, S. damnosum s.l., riverine, larval habitats. The Euclidean direction output raster contained the azimuth direction from each grid cell centroid to the nearest source. The Euclidean Allocation function identified the nearest human habitation center closest to each grid cell.The distance between sampled and human habitation areas were then categorized into specific Euclidean-distance based classes (e.g., 1:0-5 km, 2: 5-10 km, and so on) (Table 1).

\section{Ecohydrological and vegetation models}

The latest version of PCI Geomatics Orthoengine ${ }^{\circledR}$ software was then used to generate a QuickBird DEM from the spatiotemporalsampled, georeferenced, canopy, shaded, S. damnosum s.l., riverine, larval habitat data. PCI software supports automatic overlay of vector arthropod-related habitat DGPS collections, geometric modeling using Toutins rigorous model, Rationale Polynomial Coefficients (RPC) models, automatic DEM generation, orthorectification and automatic mosaicking [1]. Orthoengine also offers an industry-leading variety of control sources, including manual entry, geocoded imagery, geocoded vectors, chip database, digitizing tablet or a text file (www.pcigeomatics. com). In this research, we used the DGCP's of the sampled S. damnosum s.l. habitats to orient the images to our map coordinate system. Our RPC method used an empirical/statistical model developed by DigitalGlobe (DigitalGlobe) which approximated a 3-D physical sensor model of QuickBird. Since bias or error may still have existed in the RPCs, the results were post-processed with a polynomial adjustment product using the DGPS seasonal-sampled, canopy, shaded, riverine, larval habitats. The original RPC parameters were refined with linear equations and the DGPS data. We used:

$\Delta \mathrm{P}=\mathrm{A}_{0}+\mathrm{AS}+$ Sample + AL.Line + ASL.Sample.Line $+\ldots$.

\begin{tabular}{|l|l|l|}
\hline Variable & Description & Units \\
\hline GCP & Ground control points & Decimal-degrees \\
\hline FIOW & flowing wate & Presence or absence \\
\hline HGHT & Height of water & \\
\hline TURB & Turbidity of water & Formazin Turbidity Unit \\
\hline AQVEG & Aquatic vegetation & Percentage \\
\hline HGVEG & Hanging vegetation & Percentage \\
\hline DDVEG & Dead vegetation & Perecentage \\
\hline RCKS & Rocks & Percentage \\
\hline MMB & Man-made barriers & Type (e.g., damns, bridges) \\
\hline DISHAB & Distance between habitats & meters \\
\hline
\end{tabular}

Table 1: Environmental predictor variables sampled of the S. damnosum s.l. capture point habitat at the Dienkoa riverine study site. 
Citation: Jacob B, Novak RJ, Toe L, Sanfo MS, Caliskan S, et al. (2013) Unbiasing a Stochastic Endmember Interpolator Using ENVI Object-Based Classifiers and Boolean Statistics for Forecasting Canopied Simulium damnosum s.I. Larval Habitats in Burkina Faso. J Geophys Remote Sensing 2: 109. doi:10.4172/2169-0049.1000109

Page 19 of 36

$\Delta \mathrm{R}=\mathrm{B}+\mathrm{BS}+$ Sample+BL.Line+BSL.Sample.Line $+\ldots$.

where $A_{0}, A S, A L, A S L \ldots$ and $B_{0}$ BS BL and BSL... were the QuickBird image adjustment parameters. Since bias or error may have still existed after applying the RPCs, the results were post-processed. The original RPC parameters were refined with linear equations and the DGPS. The $\Delta \mathrm{P}$ and $\Delta \mathrm{R}$ were the adjustable functions expressing the difference between the measured and the nominal line and the sample coordinates. For most images, zero-order polynominal adjustment (e.g., $\mathrm{A}_{0}$ and $\mathrm{B}_{0}$ ) are required (www.digitalglobe.com). The OrthoEngine software supported both zero and first order RPC polynomial adjustments. A three-dimensional model of the study site was then constructed (Figure 3).

The different modules in Spatial Analyst extension of ArcGIS $10.1^{\circledR}$ was used along with spatial modeller tools from ERDAS Imagine $9.1^{\circledR}$ to perform VI calculations. NDVI was calculated using radiance, surface reflectance $(p)$, and apparent reflectance values in the QuickBird red (0.63 to $0.69 \mu \mathrm{m})$ and NIR (0.76 to $0.90 \mu \mathrm{m})$ spectral bands. The ratio of reflected radiance from the QuickBird red and NIR bands were used to normalize illumination and topographic variation and to form the NDVI, which was then used as an indicator of the amount and vigour of vegetation in the riverine epidemiological study site.

Initially, a sensitivity analysis was conducted prior to generating NDVI parameters by analyzing the atmospheric and soil-perturbed responses as a continuous function of plant Leaf Area Index (LAI). Leaf area index (LAI) is the total one-sided area of leaf tissue per unit ground surface area [1]. LAI is a key parameter in ecophysiology, especially for scaling up the gas exchange from leaf to canopy level, which normally characterizes the canopy-atmosphere interface, where most of the energy fluxes exchange. LAI can be determined directly by taking a statistically significant sample of foliage from a plant canopy, measuring the leaf area per sample habitat plot and dividing it by the plot land surface area [2]. Analysis of the literature shows that most cross-validations between direct and indirect methods have pointed to a significant underestimation of (LAI) especially in forest stands [7]. The two main causes for canopy, shaded, spectral discrepancy is clumping and contribution of stem and branches however, recent theoretical and technical solutions have been presented as potential improvements to reduce bias or discrepancies [7]. The accuracy, sampling strategy and spatial validity of the (LAI) measurements has to be constantly assessed for quality assurance of both the measurement and for modeling purposes for all calculated (LAI) -dependent ecophysiological and biophysical processes of seasonal, arthropod-related, larval habitat canopies [1]. Canopy geometry is directly related to LAI. As such, the indicator plays an essential role in theoretical production ecology [8].

In this research, LAI was used to generate photosynthetically, active S. damnosum s.l., riverine, larval habitats canopy, shade-related, explanatory, predictor, covariate, coefficient estimates for quantitating vegetation related LULC parameters at the epidemiological study site. An inverse exponential relation between LAI and light interception was established using $P=P_{\max }\left(1-e^{-c \cdot L A I}\right)$, where $P_{\max }$ designated the maximum primary production and $\mathrm{c}$ designated a vegetation specific growth coefficient. Physiological processes such as photosynthesis, transpiration and evapotranspiration are related to LAI [16]. Randomly selected grid cell and spectral measurements were assessed to determine plant LAI in ArcMap ${ }^{\circledR}$. Understanding the relation between spectral response and LAI and gridded land cover data has allowed for the modeling of canopy photosynthesis and evapotranspiration in a synoptic and repeatable fashion for identifying malaria [12], West Nile Virus WNV [13] and Eastern Equine Encephalitis Virus EEEV [14] mosquito larval habitats. Estimations of LAI production were conducted by correlation analysis with spectral reflectance ratio and measured values. The best fitting waveband ratio among calculated reflectance and VI's were selected. Percent relative error and vegetation equivalent 'noise' (VEN) were calculated for soil and atmospheric influences in the study site separately and combined using calculated LAI measurements. The NDVI had a relative error of 10 percent and VEN of \pm 0.92 LAI.

We then performed Raster modeling in ArcGIS 10.1 which included performing image differencing on the NDVI layers, classifying the layers into different $\mathrm{c}$ lasses a nd calculating a w etness index employing the Raster Calculator. The difference of the QuickBird visible and NIR bands was divided by their sum, which formed the functionally equivalent NDVI over the terrestrial surfaces of the riverine epidemiological study site. NDVI spectral variability (i.e., standard deviation of sub-grid pixel estimates) was able to differentiate multiple LULCs (Figure 4). NDVI parameters, in this research, was computed directly without any bias or assumptions regarding plant physiognomy, land cover class, soil type, or climatic conditions, within a range from -1.0 to 1.0 using the QuickBird visible and NIR reflectance, $(p)$, utilizing and the expression:

$$
\begin{aligned}
& \text { 1) } N D V I=\frac{\rho_{\text {NIR }}-\rho_{\text {red }}}{\rho_{\text {NIR }} \rho_{\text {red }}} \\
& u_{\text {cal }}^{2}(N D V I)=\left(\frac{\partial N D V I}{\partial \rho_{N I R}}\right)^{2} u_{c a l}^{2}\left(\rho_{N I R}\right)+\left(\frac{\partial N D V I}{\partial \rho_{\text {red }}}\right) u_{\text {cal }}^{2}\left(\rho_{\text {red }}\right)+2 \frac{\partial N D V I}{\partial \rho_{N I R}} \frac{\partial N D V I}{\partial \rho_{\text {red }}} \bullet u_{\text {cal }}\left(\rho_{\text {NIR }}, \rho_{\text {red }}\right) \\
& \frac{\partial N D V I}{\partial \rho_{N I R}}=\frac{2 \rho_{\text {red }}}{\left(\rho_{\text {NIR }}+\rho_{\text {red }}\right)^{2}} \\
& \frac{\partial N D V I}{\partial \rho_{\text {red }}}=\frac{-2 \rho_{N I R}}{\left(\rho_{N I R}+\rho_{\text {red }}\right)^{2}} \\
& \frac{\partial N D V I}{\partial \rho_{N I R}} \frac{\partial N D V I}{\partial \rho_{\text {red }}}=\frac{-4 \rho_{\text {NIR }} \rho_{\text {red }}}{\left(\rho_{\text {NIR }}+\rho_{\text {red }}\right)^{4}}
\end{aligned}
$$

To assess the accuracy of the QuickBird NDVI thematic maps, a simple random sampling method was chosen to ensure sampled selection. To provide a statistically efficient assessment of accuracy, a conservative sampled size equation was then employed to calculate the sample sizes using an error matrix.

For each mapping region, stratified sampling formulas were applied to estimate spectral error proportions in the ArcGIS vegetation-related LULC maps generated using the estimates of overall and class-specific user's and producer's accuracy. The use of stratified formulas is important for validating field-sampling methods. For example, stratified formulas have been extensively employed for validating QuickBird-derived malaria, WNV and EEEV -derived LULC -oriented maps [2]. Accuracy results were computed through weighting the cell proportions by the proportion of each QuickBird classified LULC within a given region. Specifically, the overall accuracy $(\widehat{P})$ and producer's accuracy $\left(\widehat{P}_{A i}\right)$ was estimated using post -stratified formulas. We used post-stratified estimators as the known QuickBird pixel totals for each land-cover class $(\mathrm{Ni}+)$ were treated as a stratified random sample of ni+ pixels from the $\mathrm{Ni}+$ pixels in that class. In this research user's accuracy $\left({ }_{U i}\right)$ were based on the random sampling formulas:

$$
\begin{aligned}
& \hat{P}=\frac{1}{N} \sum_{k=1}^{q} \frac{N_{k+}}{n_{k+}} n_{k k} \\
& \hat{P}_{U i}=n_{i i} / n_{i+}
\end{aligned}
$$




$$
\hat{P}_{A j}=\frac{\left(N_{j+} / n_{j+}\right) n_{j j}}{\sum_{k=1}^{q}\left(N_{k+} / n_{k+}\right) n_{k j}}
$$

The producer's accuracy was calculated to determine the probability that a reference sampled QuickBird-classified LULC correctly mapped and measured the errors of omission (1-producer's accuracy). In contrast, the user's accuracy indicated the probability that a sample from the QuickBird S. damnosum s.l. habitat land cover map actually matched the reference data and measured the error of commission (1-use's accuracy). Kappa statistics were then calculated using SAS PROC FREQ. Kappa statistics $(\kappa)$ are appropriate for testing whether agreement exceeds chance levels for binary and nominal ratings for remotely-sensed models constructed from spatiotemporal-sampled, spectrally-dependent, georeferenced, seasonal, vector, arthropod-related, larval habitat explanatory predictor variables [2]. The equation for $\kappa$ was:

$$
\kappa=\frac{\operatorname{Pr}(a)-\operatorname{Pr}(e)}{1-\operatorname{Pr}(e)} \text { where } \operatorname{Pr}(a) \text { was the relative observed agreement }
$$

among the uncertainty based statistics and $\operatorname{Pr}(e)$ was the hypothetical probability of chance agreement between the DGPS-sampled, georeferenced, observational, S. damnosum s.l., habitat data. In our research if the residual estimates were in complete agreement then $\kappa=1$. If there was no agreement among the classifi $\mathrm{d}$ data other than what would be expected by chance, then $\kappa \leq 0$.

\section{Object-oriented classification}

Once a robust dataset of the remotely-dependent, explanatory, predictor, covariate coefficients was constructed, ENVI ${ }^{\circledR}$ spectral tools were used to analyze the QuickBird data of the canopy, shaded, $S$. damnosum s.l., larval habitat. We input the data into $\mathrm{ENVI}^{\circledR}$ technology using the GeoTIFF format. ENVI supports Input File QuickBird functions including GeoTIFF, NITF and QuickBird Tile Product (.til) (www.ittvis.com/portals/0/pdfs/envi/ Getting_Started_with_ENVI. pdf). In ENVI $4.6^{\circledR}$, a spectrum plot, known as a $z$-profile, of the pixel under the cursor was run through all bands of the QuickBird image. The basic workflow involved importing the data collected in the field from the riverine epidemiological study site into a spectral library. The library was then employed in the endmember collection workflow to perform a supervised classification, based on the sampled $S$. damnosum s.l. riverine larval habitat spatial data feature attributes. Binary Encoding, Spectral Angular Mapping (SAM) and Spectral Feature Fitting were used to rank and match any unknown spectrum to the materials in the library.

The endmembers of the georeferenced canopy shade-related $S$. damnosum s.l. riverine larval habitat and its associated attributes were then extracted them from ENVI ${ }^{(\mathbb{B})}$ s spectral library. Several spectra corresponding to the different backgrounds in the canopy, shaded, habitat structures (i.e., Precambrian rock and rippled water pixel components) had to be included, since multiple scatterings between floating leaves in the habitat, and a bright soil background increased the QuickBird NIR reflectance. Leaf cells have evolved to scatter (i.e., reflect and transmit) solar radiation in the NIR spectral region [8]. After the calibration estimates had been determined the image was converted to match the library. Analogously, the QuickBird reference endmembers spectra in the library were transformed into the endmembers spectra of the image. In this research we used a random selection of pixels to assess the satellite classification accuracy. QuickBird class representative pixels were thereafter selected and compared to a reference training dataset. During the segmentation procedure, image objects were generated based on several adjustable criteria of homogeneity such as colour, shape, and texture. Data preprocessing involved converting the $\mathrm{DN}$ to radiance atmospheric correction using $\mathrm{FLAASH}^{\mathrm{TM}}$, and co-registration techniques. Image classification was conducted thereafter using the object-oriented approach. FLAASH ${ }^{\mathrm{TM}}$ generated a model (.gmd file) that converted the image's DN to at-sensor radiance and computed at-sensor reflectance while normalizing the solar elevation angle. The equation was as follows:

$$
\rho \text { Band } N=\frac{\pi(\text { LBandN } * \text { GainBand } N+\operatorname{BiasBand} N) * D^{2}}{\operatorname{EBand} N *(\operatorname{COS}((90-\theta) * \pi / 180))}
$$

where,

$\rho_{\text {BandN }}=$ Reflectance for Band $N$

$\mathrm{L}_{\text {bandN }}=$ Digital Number for Band $\mathrm{N}$

$\mathrm{D}=$ Normalized Earth-Sun Distance

$$
\mathrm{E}_{\text {bandN }}=\text { Solar Irradiance for Band } \mathrm{N}
$$

The reference data in this research was the "ground truthed" data of the selected canopy, shaded, S. damnosum s.l., riverine, larval habitat, explanatory, predictor variables. Selected random pixels from the thematic map were then compared to the reference data. The estimated accuracy for the spectrally extracted data was calculated: $P\left\{-z_{\frac{\alpha}{2}}<\frac{x-n \theta}{\sqrt{n \theta(1-\theta)}}<z_{\frac{\alpha}{2}}\right\}=1-\alpha$ where, $x=$ number of correctly identified pixels, $n=$ total number of pixels in the sample, $\theta=$ the map accuracy, $(1-\alpha)=a$ confidence limit. The QuickBird classifier identified the canopy shaded habitats and their associated Precambrian rock and ripple water larger than 0.4 has with generally satisfactory results (92.1\%) with a lower detection limit of 0.1 ha.

\section{The Successive Projection Algorithm (SPA)}

We employed an SPA to generate robust, canopy, shaded, endmembers from a georeferenced, S. damnosum s.l., riverine, larval habitat, the Precambrian rock and the ripple water spectra (i.e., capture point). We let $\mathrm{P}(i, j)$ denote the spectrum for the extracted QuickBird pixel data using the image coordinates $(i, j)$, as the foundation of linearlybased spectral unmixing algorithm which in this research was defined by $\vec{p}_{(i, j)}=\sum_{k=1}^{m} f_{(i, j) k} \vec{e}_{k}+\vec{\varepsilon}_{(i, j)}$ and also $f(i, j) k \geq 0, k=1, \ldots, m, \sum_{k=1}^{m} f_{(i, j) k}=1$, where $m$ was the number of endmembers, $\vec{e}_{k}$ was the $k t h$ end member, $\vec{\varepsilon}_{(i, j)}$ was the approximation error term (residual), and $f(i, j) k$, was the fractional abundance for the $k$ th endmember of sub-pixel components $(i, j)$.

We omitted the error term in $\vec{p}_{(i, j)}=\sum_{k=1}^{m} f_{(i, j) k} \vec{e}_{k}+\vec{\varepsilon}_{(i, j)}$ and computed the possible linear mixtures from $\vec{p}_{(i, j)}=\sum_{k=1}^{m} f_{(i, j) k} \vec{e}_{k}+\vec{\varepsilon}_{(i, j)}$ and $f(i, j) k \geq 0, k=1, \ldots, m, \sum_{k=1}^{m} f_{(i, j) k}=1$ which formed a simplex $C m$ defined by $m$ vertices that corresponded to the canopy, shaded $S$. damnosum s.l., riverine, larval habitat endmembers, $\vec{e}_{1}, \vec{e}_{2}, \ldots, \vec{e}_{m}$. The volume of the simplex $\mathrm{Cm}$ was then calculated from the equation $V\left(C_{m}\right)=\frac{1}{(m-1) !}\left|\operatorname{det}\left(W W^{T}\right)\right|^{1 / 2} \quad$ where $W=\left[\vec{e}_{2}-\vec{e}_{1}, \vec{e}_{3}-\vec{e}_{1}, \ldots ., \vec{e}_{n}-\vec{e}_{1}\right] \quad$ was the volume of the simplex defined by $m$ endmembers, and where $\operatorname{det}(\cdot)$ denoted the determinant of a matrix representing the operation of an absolute value. In this research, once the endmembers $\vec{e}_{1}-\vec{e}_{2}, \ldots, \vec{e}_{m}$ were determined, their abundance was estimated through the least squares method which was equivalent to a projection on the simplex. 
Citation: Jacob B, Novak RJ, Toe L, Sanfo MS, Caliskan S, et al. (2013) Unbiasing a Stochastic Endmember Interpolator Using ENVI Object-Based Classifiers and Boolean Statistics for Forecasting Canopied Simulium damnosum s.l. Larval Habitats in Burkina Faso. J Geophys Remote Sensing 2: 109. doi:10.4172/2169-0049.1000109

Page 21 of 36

Employing this framework, all the georeferenced, canopy, shaded S. damnosum s.l., riverine, larval habitat data points based on the QuickBird pixel encompassing the Precambrian rock and rippled water components were examined in $\mathrm{n}$-dimensional space. Endmembers present in the scene were found at the vertices of the simplex. The interior space of the simplex represented feasible mixtures. The task of finding robust endmembers associated to the S. damnsoum s.l. riverine habitat the Precambrian rock and the ripple water reflectance was actually the identification of the simplex vertices, which in this research was the foundation for the interpretation of the decomposed QuickBird sub-mixel spectral information based on a convex geometrical endmember extraction algorithm.

A meaningful endmember spectra for the vertex was then calculated using the vector Euclidean norm to the subspace as defined by selected capture point canopy shaded endmembers. To find these candidate sub-pixel attributes, we constructed a QuickBird endmember dataset using, $P$ possible consisting of $r$ spectral emissivities that were closest to the vertex. By so doing, we generated a canopy, shaded, $S$. damnsoum s.l., larval habitat spectral endmember subset using

$P_{\text {candidate }}=\left\{\vec{p}\left(x_{1}, y_{1}\right), \vec{p}\left(x_{1}, y_{1}\right), \ldots, \vec{p}\left(x_{c}, y_{c}\right)\right\}\left(c \leq r, P_{\text {candidate }} \in P_{\text {possible }}\right)$ which was then selected from a candidate mixel $r\left(P_{\text {possible }}\right)$ which was subject to conditions based on: $x_{i}-x_{j} \leq t_{-}$pixel and $y_{i}-y_{j} \leq t_{-}$pixel $(i \neq j)$. We used the equation $s_{-} \operatorname{angle}\left(\vec{p}_{\left(x_{i}-y_{i}\right)}, \vec{p}_{\left(x_{j}, y_{j}\right)}\right) \leq t_{-} \theta(i \neq j)$ as the spectral angle between the georeferenced $S$. damnosum s.l. habitat, Precambrian rock and ripple water sub-pixel radiance and calculated $s_{-} \operatorname{angle}\left(\vec{p}_{\left(x_{i}-y_{i}\right)}, \vec{p}_{\left(x_{j}, y_{j}\right)}\right)=\cos ^{-1}\left(\frac{\vec{p}_{\left(x_{i}-y_{i}\right)} \cdot \vec{p}_{\left(x_{j}, y_{j}\right)}}{\left|\vec{p}_{\left(x_{i}-y_{i}\right)} \| \vec{p}_{\left(x_{j}, y_{j}\right)}\right|}\right)$, where $t \_\theta$ was the threshold value for the spectral angle beyond which the QuickBird endmember spectra were not considered similar. The value of $t \_\theta$ was set at 2.5 degrees. The SPA identified $\vec{e}_{1}, \vec{e}_{2}, \ldots, \vec{e}_{m}$ which represented the brightest sub-pixel data (ripple water white endmember components) and the darkest sub-pixel data (black Precambrian rock). The algorithm then iteratively found the remaining endmembers using orthogonal projections. Thereafter all the canopy, shaded-related endmembers were identified.

A number of Precambrian rock and ripple water canopy, shade, related, endmember spectral components $(m)$ were used to find the spectral angle threshold $t_{-} \theta$ and the spatial threshold (i.e., $t_{-} \theta$ QuickBird pixel). The next step was to extract the first endmember. The vector norms of the QuickBird data determined the largest norm value. Sub-pixel heterogeneity at the simplex vertices was then calculated which revealed the entire mixel data in the image cube. The S. damnosum s.l., riverine habitat first canopy, shade-related, endmember were then estimated.

We employed an orthogonal projection for extraction of all the $S$. damnosum s.l. habitat, Precambrian rock and ripple water sub-pixel, canopy, shade-related, endmember components. All the QuickBird spectral data was fractionally calculated based on the visible and NIR

spectrum. We used an endmember matrix $U=\left[\vec{e}_{1}, \vec{e}_{2}, \ldots, \vec{e}_{m}\right]$ which projected all the QuickBird data into subspace $S_{\text {proj }}$ orthogonal to the space spanned by $U$ as $\vec{p}_{(i, j) \_ \text {proj }}=O \vec{p}_{(i, j)}$, where $\vec{p}_{(i, j) \_ \text {proj }}$ and $\vec{p}_{(i, j)}$ were the projected and original pixel vector at the $S$. damnosum s.l. habitat location $(i, j)$ respectively. In this research, $O$ was the projection operator, $O=I-U U^{+}$where $I$ was the identity matrix and $U^{+}$was the pseudo inverse of $U$ which we denoted was $U^{+}=\left(U^{T} U\right)^{-1} U^{T}$. We then validated the endmember matrix (i.e., $U=\left[\vec{e}_{1}, \vec{e}_{2}, \vec{e}_{3}\right]$ ). We calculated the change of the simplex volume with each subspace projection. The volume of the simplex was then calculated. The volume increase was determined by the spectral contrasts between the canopy endmembers. In this research, $C_{l-1}$ and $C_{l}$ denoted the simplexes defined by the original endmember set, $\left\{\vec{e}_{1}, \vec{e}_{2}, \ldots, \vec{e}_{l-1}, \vec{e}_{1}\right\}$ and the ratio of the volumes of $C_{l-1}$ and $C_{l}$ was calculated as $v_{-}$ratio $l=\frac{V\left(C_{l}\right)}{V\left(C_{l-1}\right)}$.

\section{Canopy Radiative Transfer Equation}

For spectrally quantitating the canopy layer, we used a 3-D, canopy, S. damnosum s.l., riverine, larval habitat object scene. The shaded canopy was modeled as a combination of geometric shapes (e.g., cylinders and ellipsoids). The georeferenced larval habitat leaf area density (u) and branch area density (b) within the single canopy were assumed to be spatially non-uniform. Understory vegetation was then modeled as a plane parallel layer. In the canopy layer, we assumed that photons interacted with vegetation media. Optical properties, such as the extinction coefficient for unit volume ( $\beta \mathrm{cnp}, \mathrm{k})$, single scattering albedo of a single leaf $(\omega \mathrm{cnp}, \mathrm{k})$ and phase function (Pcnp,k) were then used as optical properties in the kth canopy, shaded, larval habitat computations. $\beta \mathrm{cnp}, \mathrm{k}$ was expressed as a product of the $\mathrm{G}$ and leaf area density (uk). The $\mathrm{G}$ was the fraction of average projected leaf area along the direction of photon propagation $(\Omega)$ which was quantitated by

$$
G(\Omega)=\frac{1}{2 \pi} \int_{0}^{2 \pi} \int_{0}^{\frac{\pi}{2}} g_{L}\left(\Omega_{L}\right)\left|\Omega_{L} \cdot \Omega\right| \mathrm{d} \Omega_{L} \mathrm{~d} \theta_{L} \mathrm{~d} \varphi_{L}
$$

using a leaf angle distribution function and a normal vector of the leaf surface, respectively. In our canopy, shaded, S. damnosum s.l., riverine, larval habitat model schemes, all shapes of gL used i. The uniform leaf angle distribution was assumed in all calculation in this study (i.e., $G=0.5$ ). The integration of uk along the vertical direction is commonly known as LAI [1]. $\omega \mathrm{cnp}$, $\mathrm{k}$ was then expressed as the sum of single leaf reflectance (rk) and transmittance (tk) $\omega \mathrm{cnp}, \mathrm{k}=\mathrm{rk}$ $+\mathrm{tk} . \omega \mathrm{cnp}, \mathrm{k}=\mathrm{rk}+\mathrm{tk}$. The phase function of the kth canopy component (Pcnp,k) was then expressed as

$$
=\frac{P_{c n p, k}\left(\Omega^{\prime}, \Omega\right)}{\left.\omega_{c n p, k} G\left(\Omega_{L}\right)\left|\Omega^{\prime} \cdot \Omega\right| p_{L, K}\left(\Omega^{\prime},\right) \Omega, \Omega_{L}\right) \mathrm{d} \Omega_{L}} \text { were the photon vectors were }
$$

quantitated before and after the scattering event, respectively. $\mathrm{pL}, \mathrm{k}$ is a single leaf phase function for the kth canopy given the normal of the leaf $(\Omega \mathrm{L})[1]$. Scattering within the riverine larval habitat canopy was then also calculated. Lambertian was assumed for scattering on soil and stem surface in the model.

The model started the tracing of the photon with initial weight $\left(\mathrm{w}_{0}=1\right)$ from the TOA. To determine the photon traveling path length (s), optical thickness $\tau$ in the atmosphere and the S. damsnoum s.l. riverine larval habitat canopy was first determined using a random number (R) where $\tau=-\ln$ and $\tau=-\ln$. A scattering event can occur at $R$, where the optical thickness that is integrated along the photon traveling path is equal to $\tau$ in the above equation [45]. The new photon direction $\Omega$ $(\theta, \phi)$ after scattering was then determined in the model. We noticed that when $\mathrm{P}$ was dependent only on the scattering angle [e.g., a $\left(=\cos -1\left[\Omega^{\prime} \cdot \Omega\right]\right)$. The shaded canopy revealed uniform leaf angle distribution. The $\alpha$ was then determined by: $\frac{1}{2} \int_{0}^{\dot{a}} P\left(\alpha^{\prime}\right) \sin \alpha^{\prime} \mathrm{d} \alpha^{\prime}=R$. where $12 \int 0 \alpha \mathrm{P}\left(\alpha^{\prime}\right)$ when $d \mathbb{d} \alpha^{\prime}=\mathrm{R}$. In this research the azimuth angle relative to the scattering coordinate $\phi$ was determined randomly. 
The scattering direction $\Omega(\theta, \phi)$ in the cartesian coordinate system was calculated by a coordinate transformation from $\Omega s(\alpha, \phi s)$ to $\Omega(\theta, \phi)$. The relationship between the random number $\mathrm{R}$ and the prescribed scattering angle $(\alpha)$ in the lowest atmospheric layer and in the larval habitat canopy foliage was then spectrally quantitated.

Next, the function of the atmosphere was calculated for the following conditions: atmospheric profile: mid-latitude summer, aerosol: optical thickness: 0.3 , cloud: stratus continental and optical thickness. The function of the canopy was calculated by the following condition: $\rho=0.2, \tau=0.1$ with uniform leaf angle distribution $(G=0.5)$.

When a periodic condition is assumed for horizontal boundaries, the outgoing photon from the lateral face re-enters from the opposite side of the face with same photon direction and weight [1]. This then enabled us to easily calculate APAR (i.e, absorbed PAR) in the riverine larval habitat shaded canopy. After the jth interaction the weight of a non-absorbed part of photon became $w j+1=w j \omega w j+1=w j \omega$.

For the reduction of the computation time, the photon with a low weight was randomly cut off using the "Russian roulette" $t$ echnique in the model. This unbiased technique is widely used in canopy and atmospheric radiative transfer modeling [1]. In this technique, traditionally, when the weight of the photon becomes smaller than the given threshold $(\varepsilon)$, the photon is cut off by the given probability $(1-\mathrm{q})$. When the photon survives with a probability $\mathrm{q}$, the weight of the photon is modified following w/q so as not to give rise to artificial biases.

In this simulation, we employed the $5 \%$ and $10 \%$ values of the canopy, shaded, S. damnosum s.l., riverine, larval habitat average $\omega \mathrm{cnp}, \mathrm{k}$ over all canopy as a threshold $(\varepsilon)$ and survival probability (q). Under these conditions, "Russian roulette" was then applied after approximately third order scattering in visible region $(\omega \mathrm{cnp}, \mathrm{k} \approx 0.1$ ) and after less than tenth order scattering in NIR region (i.e., $\omega \mathrm{cnp}, \mathrm{k} \approx$ $0.8)$.

Because light environmental parameters were sampled at each scattering event, sufficient sampling was not expected in such a small leaf area density case. The single scattering albedo and phase function were then adjusted to be invariant before and after the scaling. While the method was originally developed for the atmospheric radiative transfer problem with optically thin media, it is directly applicable for 3-D canopy radiative transfer modeling [15]. Because the leaf area density was small in our model we scaled the $\beta \mathrm{cnp}, \mathrm{k}$ to $\beta^{\prime} \mathrm{cnp}, \mathrm{k}$ $\left(\beta c n p, k<\beta^{\prime} c n p, k\right)$. In radiative transfer modeling, when the photon enters the optically thin canopy object, the extinction coefficient is scaled [1].Then $\omega c n p, k$ and Pcnp were scaled as $\omega^{\prime} c n p, k=1-(1$ $-\omega c n p, k)$ fe $\omega^{\prime} c n p, k=1-(1-\omega c n p, k)$ fewhere fe was the ratio of the extinction coefficient before and after scaling $\left(\beta \mathrm{cnp}, \mathrm{k} / \beta^{\prime} \mathrm{cnp}, \mathrm{k}\right)$ and $\delta$ was a Dirac delta function. The fraction $f d$ in the canopy, shaded, $S$. damnosum s.l., riverine, larval habitat model was then expressed as:

$$
f_{d}=\frac{1-f_{e}}{\omega_{c n p, k}^{\prime}} \text { where fd=1-fewcnp, } \mathrm{k}^{\prime} .
$$

The new direction of the photon and the change in photon weight were determined by $\omega^{\prime} \mathrm{cnp}, \mathrm{k}$ and $\mathrm{P}^{\prime} \mathrm{cnp}, \mathrm{k}$. In the determination of the new direction, the random number ( $\mathrm{R}$ ) was first compared to $\mathrm{fd}$. If $\mathrm{R}<\mathrm{fd}$, the scattering direction is unchanged, whereas if $\mathrm{R}>\mathrm{fd}$, the scattering direction is determined [1]. The APAR, described every scattering event in the georeferenced, canopy, shaded, S. damnsoum s.l., riverine, larval habitat.
Sampling of APAR, and irradiance light environmental variables, such as TOC in the model, involved calculating canopy, irradiance using the QuickBird signals. By so doing, the spatial distributions of these variables in the 3-D canopy, pixel and voxelbased samplings were quantitated. When each canopy, shaded, QuickBird pixel and voxel in the riverine epidemiological study site did not overlap each other, total horizontal area (Atot) and total volume (Vtot) in the simulated landscape was expressed as

$$
A_{\text {tot }}=\sum_{l} A(l) \text { where } \mathrm{A}_{\text {tot }}=\sum \text { and } V_{\text {tot }}=\sum_{m} V(m) \cdot \text { where } V_{\text {tot }}=\sum_{m} V(m) .
$$

TOC was then sampled using a local estimation method. This method analytically calculated the contribution for BRDF af ter every scattering. The reflectance contribution factor $\Psi(\Omega 0)$ of the $j^{\text {th }}$ scattering event for the direction $\Omega$ o was then sampled using the formula $\psi_{j}\left(\Omega_{0}\right)=\frac{w_{j+1} P\left(\Omega_{j}, \Omega_{0}\right) \exp \left(-\tau^{\prime}\right)}{4 \pi\left|\cos \theta_{0}\right|}$.

Here, P indicated either Patm, $\mathrm{k}$ or Pcnp, $\mathrm{k}$, was dependent on the media at scattering point. $\tau^{\prime}$ which was a modified optical thickness for the hotspot effect. However, this interaction was dependent on the strong backscattering effect near the solar direction (i.e., $\Omega s=-\Omega o$ ). To include the hotspot effect in the $S$. damnosum s.l. canopy scattering, we adjusted original optical thickness $\tau$, which was the optical distance from the scattering point (i.e., rsca) to the TOC point. In this research these larval habitat spectral properties were quantitated using the hotspot function $(\mathrm{H})$ employing

$$
\tau^{\prime}=\tau H\left(\Omega_{j}, \Omega_{0}\right)(0 \leq \mathrm{H} \leq 1) .
$$

Various types of hotspot function are available if they are normalized in [0-1] [1]. We used a simple approximation of the hotspot function proposed by Hapke (1994) who employed

$$
H\left(\Omega_{j}, \Omega_{0}\right) \cong 1-\frac{1}{\left(1+\frac{1}{h\left(\Omega_{j}, \Omega_{0}\right)} \tan (\alpha / 2)\right)}
$$

where h was expressed as $h\left(\Omega_{j}, \Omega_{0}\right) \cong \frac{u_{k} l_{k}}{2}\left(\frac{G\left(\Omega_{j}\right)+G\left(\Omega_{0}\right)}{2}\right)$. TOC of the $\mathrm{l}^{\text {th }}$ pixel in the simulated riverine landscape was then calculated by summing all contributing factors: $B R F\left(l, \Omega_{0}\right)=\frac{\pi}{n A(l)} \sum_{i=1}^{N} \sum_{j=1}^{M_{1}}\left\{\begin{array}{cc}\psi_{i, j} & \left(r_{o b s} \in A(l)\right) \\ 0 & \left(r_{o b s} \notin A(l)\right)\end{array}\right.$ where

$\mathrm{BRF}$ and $\mathrm{N}$ was the total number of photons and Mi was the number of scattering times for the ith photon, respectively. The $\mathrm{n}$ is an incident photon density at TOA $[\mathrm{m}-2][1]$. We then employed $n=\frac{N}{A_{t o t}}$. where
$\mathrm{n}=$ NAtot.

We calculated the angular variation of downward diffuse radiance incident at TOC. In this case, $\tau^{\prime}$ was an atmospheric optical thickness from the scattering point to TOC, and $\pi$. We then defined the downward spectral solar flux at TOA (i.e., F $\lambda$, [W $\mathrm{m}-2 \mathrm{~nm}-1$ or $\mu \mathrm{mol} \mathrm{m-2} \mathrm{s-1} \mathrm{nm-1]).} \mathrm{In} \mathrm{this} \mathrm{research,} \mathrm{APAR} \mathrm{[W} \mathrm{m-3} \mathrm{or} \mu \mathrm{mol} \mathrm{m-3}$ $\mathrm{s}-1$ ] of the $\mathrm{m}^{\text {th }}$ voxel was calculated by simply sampling the change in photon energy in every scattering event occurring within the voxel $\begin{array}{ll}\text { using: } & F_{\text {par }} \\ n \text { AR }(m) & N\end{array} \sum_{i=1}^{N} \sum_{j=1}^{M_{1}}\left\{\begin{array}{cc}w_{i, j}\left(1-\omega_{c n p, k}\right) & \left(r_{\text {sca }} \in V(m)\right) \\ 0 & \left(r_{\text {sca }} \notin V(m)\right)\end{array},\left(F_{p a r}=\int_{400}^{700} F_{\lambda} d_{\lambda}\right)\right.$.

By so doing, we found that APAR for the canopy, shaded, S. damnosum s.l. riverine, larval habitat. The APAR of the direct irradiance (APARdir) was then evaluated by counting only the contribution from the first 
Citation: Jacob B, Novak RJ, Toe L, Sanfo MS, Caliskan S, et al. (2013) Unbiasing a Stochastic Endmember Interpolator Using ENVI Object-Based Classifiers and Boolean Statistics for Forecasting Canopied Simulium damnosum s.l. Larval Habitats in Burkina Faso. J Geophys Remote Sensing 2: 109. doi:10.4172/2169-0049.1000109

Page 23 of 36

order scattering (i.e., $j=1$ ), and the APAR of the diffuse irradiance (i.e., APARdif) was evaluated by neglecting the contribution from the first order scattering $(j=1)$.

Downward irradiance of the $\mathrm{l}^{\text {th }}$ pixel in horizontal plane at height $\mathrm{z}[\mathrm{m}], \mathrm{Iz}(\mathrm{l})$, was calculated by counting the photon weight that passed through the horizontal plane using

$$
I_{z}(l)=\frac{F_{\lambda}}{n A(l)} \sum_{i=1}^{N} \sum_{j=1}^{M_{1}}\left\{\begin{array}{cc}
\psi_{i, j} & \left(r_{z} \in A(l)\right) \\
0 & \left(r_{z} \notin A(l)\right)
\end{array} .\right.
$$

As well the irradiance was calculated when the S. damnosum s.l. riverine habitatpoint $r_{z}$ was located in the QuickBird pixel with A (l).

The sunlit leaf area density in a unit voxel (us [m-1]) was calculated by counting the number of photons from the first scattering employing $\quad u_{s}=\frac{1}{G\left(\Omega_{j=0}\right) n V(m)} \sum_{i=1}^{N}\left\{\begin{array}{ll}1 & \left(r_{s c a} \in V(m)\right) \\ 0 & \left(r_{s c a} \notin V(m)\right)\end{array}\right.$. In this equation the canopy, shaded, S. damnosum s.l., weighted, explanatory covariates were only deemed worthwhile if after incorporation into the model the first scattering occurred in the voxel.

Spectral integration of our model output was then conducted by integrating the sampled canopy, shaded, S. damnosum s.l., riverine, larval habitat, predictor variables over a spectral region, such as PAR using the calculated ranges. The number of photon in each waveband $(\Delta \lambda)$ needed to be determined by their weight to tabulate proportionality to $\mathrm{F} \lambda$. When $\mathrm{N}$ photons are used for the radiative transfer calculation in the spectral ranges $\left[\lambda_{\min }, \lambda_{\max }\right]$, the number of photons $(\mathrm{Ni})$ in the $\mathrm{i}^{\text {th }}$

waveband $[\lambda \mathrm{i}, \lambda \mathrm{i}+1]$ is $N_{i}=N \frac{\int_{\lambda i}^{\lambda i+1} F_{\lambda} \mathrm{d} \lambda}{\int_{\lambda \min }^{\lambda \min } F_{\lambda} \mathrm{d} \lambda},\left(\sum N_{i}=N\right)$ where the weight and energy of single photon is the same in spectral calculations [1].

The light environmental variables calculated from the radiative transfer simulation were then directly used for canopy photosynthesis simulation. We simulated canopy photosynthesis based on Farquhar's leaf photosynthesis model. In the calculation, we ignored the spatial heterogeneity of the light environment within a single voxel to simplify the calculation. Two representative leaves (i.e., sunlit leaf and shaded leaf) were then employed for the single voxel photosynthesis calculation. Diffuse light was assumed to be incident from the upper hemispherical direction as diffuse PAR contribution from downward hemisphere is small [17]. Under these assumptions, photosynthesis in a single voxel $(\mathrm{PSN}(\mathrm{m}))$ was expressed as a summation of photosynthesis rate from sunlit and shaded leaves using:

$$
\begin{aligned}
& \operatorname{PSN}(\mathrm{m})=\frac{u_{s}}{2 \pi} \int_{2 \pi} P S N_{\text {leaf }}\left(A P A R_{\text {leaf }, \text { sun }}\right) g_{\mathrm{L}}\left(\Omega_{L}\right) \mathrm{d} \Omega_{L}+\frac{\left(u-u_{s}\right)}{2} \\
& \int_{2 \pi} P S N_{\text {leaf }}\left(A P A R_{\text {leaf } \text {, shade }}\right) g_{L}\left(\Omega_{L}\right) \mathrm{d} \Omega_{L}
\end{aligned}
$$

where PSN leaf was a leaf photosynthesis rate and APAR leaf, sun and APAR leaf, shade were the APAR in the unit leaf area $[\mu \mathrm{mol} \mathrm{m-2}$ s-1] for sunlit and shaded leaves, respectively. We

used $\quad A P A R_{\text {leaf }, \text { sun }}=I_{\text {dir }}, T O C \times\left|\Omega_{s} \cdot \Omega_{L}\right| \times\left(1-\omega_{\text {cnp }, k}\right)+\frac{A P A R_{\text {dif }}}{u} \quad$ and $A P A R_{\text {leaf }, \text { shade }}=\frac{A P A R_{\text {dif }}}{u}$ where shade $=\mathrm{APAR}$ and where Idir, TOC $[\mathrm{W}$ $\mathrm{m}-2$ ] was the downward irradiance at TOC.

\section{Inverted geometric-optical model}

We tused the Li-Strahler geometric-optical model based on the assumption that the BRDF would retrieve the canopy, shaded, S. damnosum s.l., riverine, habitat, shaded, riverine, canopyoriented, structural photosynthetic and non-photosynthetic explanatory predictor variables. The BRDF was first defined by:

$$
f_{r}\left(\omega_{i}, \omega_{o}\right)=\frac{d L_{r}\left(\omega_{o}\right)}{d E_{i}\left(\omega_{i}\right)}=\frac{d L_{r}\left(\omega_{o}\right)}{L_{i}\left(\omega_{i}\right) \cos \theta_{i} d \omega_{i}}
$$

where $L$ was the radiance, $E$ was the irradiance, and $\theta_{i}$ was the angle made between $\omega$ i and the canopy, shaded habitat and its associated Precambrian rock and ripple water surface reflectance emissivities. Because the BRDF is a four-dimensional function that defines how light is reflected at a $\mathrm{n}$ o paque s urface [1], $\mathrm{t}$ he $\mathrm{f}$ unction $\mathrm{t}$ ook a $\mathrm{n} \mathrm{i}$ ncoming light direction, $\omega_{i}$, and outgoing direction, $\omega_{0}$, which were both defined with respect to the canopy, shaded, S. damnosum s.l., larval habitat and its neighboring Precambrian rock and ripple water surface $n$, and returned the ratio of reflected radiance exiting along $\omega 0$ to the irradiance incident from direction $\omega_{\mathrm{i}}$. Note, each direction $\omega$ was itself parameterized by azimuth angle $\varphi$ and zenith angle $\theta$. Therefore, in this research, the BRDF was 4-dimensional. The BRDF had units sr ${ }^{-1}$, with steradians (sr) being a unit of solid angle.

The inverted Li-Strahler geometric-optical model was then employed to retrieve specific photosynthetic, spectrallydependent, riverine, larval habitat, explanatory, photosynthetic and non-photosynthetic predictor, covariate, coefficient, estimates. The reflectance associated with a georeferenced habitat was treated as an area-weighted sum of four fixed reflectance components: sunlit canopy, sunlit background, shaded canopy, and shaded background. In most geometric-optical models these four components are simplified to three spectral variables: sunlit canopy-C, sunlit background- $G$ and shadow- $T$ [1]. In this research, the model employed three scene components: Precambrian Rock in sunlit canopy $(\mathrm{P})$, white sunlit turbid riffle water data $(\mathrm{T})$ and vegetation-related shadow background $(\mathrm{V})$. These components were initially estimated by the QuickBird image using $\mathrm{ENVI}^{\circledR}$, object-based classification methods.

For inverting the model, parts of the three spectral components were represented by $(\mathrm{Kg})$ which was calculated using:

$$
\begin{aligned}
& K_{g}=e^{-\pi \cdot M \cdot\left[\sec \left(\theta_{i}\right)+\sec \left(\theta_{v}\right)-O\left(\theta_{i}, \theta_{v}, \phi\right)\right]} \\
& O\left(\theta_{i}, \theta_{v}, \phi\right)=1 / \pi\left(\sec \theta_{i}+\sec \theta_{v}\right)(t-\sin t \cos t) \\
& \cos t=\frac{h\left|\tan \theta_{i}-\tan \theta_{v} \cos \phi\right|}{r\left(\sec \theta_{i}+\sec \theta_{v}\right)} \\
& M=\frac{-\ln \left(K_{g}\right)}{\left(\sec \theta_{i}+\sec \theta_{v}\right)(\pi-t+\cos t \sin t)} \\
& C C=1-e^{-\pi \cdot M}
\end{aligned}
$$

where, $I q u q$ were the zenith angles of illumination and viewing, $O$ was the average of the overlap function between illumination and viewing shadows of the canopy, shaded, S. damnosum s.l., larval habitat (i.e., capture point) and their associated Precambrian rock and ripple water components as projected onto the background. In this research, $j$ was the difference in azimuth angle between illumination and viewing.

In our analyses, the BRDF of the canopy, shaded, S. damnosum s.l., 
habitat pixel was modeled as the limit of its directional reflectance factor using

$$
R(i, v): R(i, v)=\frac{\iint_{A} R(s)\langle i, s\rangle\langle v, s\rangle I_{i}(s) I_{v}(s) d s}{A \cos \theta_{i} \theta_{v}}
$$

where $d s$ was a small Lambertian surface element over area $A$ of the QuickBird pixel; $R(s)$ was the reflectance of $d s ; i, v$, and $s$ represented the directions of illumination and viewing based on the Precambrian rock surface and ripple water reflectance components, respectively. In our model $\langle. .$.$\rangle was the cosine of the phase angle between two$ directions; $\theta$ was the zenith angle of a direction and, $I_{i}(s)$ and $I_{v}(s)$ were indicator functions, equal to one when $d_{s}$ was illuminated $\left(I_{i}\right)$ or viewed $\left(I_{v}\right)$ or zero otherwise. If a surface exhibits Lambertian reflectance, light falling on it is scattered such that the apparent brightness of the surface to an observer is the same regardless of the observer's angle of view (i.e., isotropic surface luminance) [8]. Solving our double integral equation revealed an integrated over the decomposed QuickBird pixel (i.e., the footprint of the sensor's iFOV).

In this research, there were two kinds of prominent habitat surfaces in the canopy, shaded, mixel spectra; background surface (i.e., Precambrian rock) and surface ripple water-which were represented by Lambertian reflectance $G$ and $C$, respectively. We then re-wrote equation (2.1) as

$$
R(i, v)=K_{g} G+\frac{C}{A} \iint_{A_{c}} \frac{\langle i, s\rangle}{\cos \theta_{i}} \frac{\langle v, s\rangle}{\cos \theta_{v}} d s, \text { where } K_{g}=A_{g} / A
$$

which was the proportion of background spectral data illuminated and viewed generated by the QuickBird imaged canopy shaded S. damnosum s.l. riverine habitat data spectral feature attributes. In this equation the union of $A_{\sigma}$ and $A_{e}$ were the intersection of the dataset of the canopied S. damnosum s.l. riverine larval habitat surface elements. The directional reflectance of the canopied georeferenced habitat scene depended also on the Precambrian rock and ripple water reflectance related to $G$ and $C$.

In our analyses we focused on the two terms of

$$
R(i, v)=K_{g} G+\frac{C}{A} \iint_{A_{c}} \frac{\langle i, s\rangle}{\cos \theta_{i}} \frac{\langle v, s\rangle}{\cos \theta_{v}} d s .
$$

The first term described how the sunlit background proportion proceeded to a maximum point as viewing and illumination positions in the hemisphere coincided. The second term described how the sunlit georefernced S. damnosum s.l. habitat canopy surface, composed of the Lambertian facets, became maximally exposed to view at the hotspot, while those facets on tops became dominant at large viewing zenith angles. The hot spot correlation effect refers to the observed brightening which can occur when viewing a scene from the same direction as the solar illumination, which for vector arthropod-related larval habitat geopredictive endmember endemic transmission-oriented risk modeling is commonly noted in the visible and NIR spectral regions [20].

We analyzed how the first term $K_{g} G$ varied with illumination and viewing geometry. As in [21], we assumed that the spatial object of interest (i.e., canopy, shaded, S. damnosum s.l. habitat) and its associated photosynthetic spectrally-dependent, explanatory covariate, coefficient estimates had the shape of a spheroid, with vertical half-axis equal to $b$, horizontal radius equal to $R$, and a height to the center of the spheroid $h$. To accommodate the spheroidal shape in the derivations of the shadowed habitat areas, we used the transformation

$\theta^{\prime}=\tan ^{-1}\left(\frac{b}{R} \tan \theta\right)$. We solved this equation by replacing $\theta$ with the angle that would generate the same shadow area for a sphere. For simplicity, we assumed that the centers of the spheroids were randomly distributed in depth from $h_{1}$ to $h_{2}$ over $A$. We then assumed that $G$ and $C$. We then assumed that and were constant as average signatures over $A_{g}$ and $A_{c}$ for properly defining $K_{g}$ and $K_{c} \neq A$

$$
\text { Next, the equation } R(i, v)=K_{g} G+\frac{C}{A} \iint_{A_{c}} \frac{\langle i, s\rangle}{\cos \theta_{i}} \frac{\langle v, s\rangle}{\cos \theta_{v}} d s
$$
employed where by $K_{g}$ was expressed in a Boolean model and $K_{g}=e^{-\lambda \pi R^{2}\left[\sec \theta_{i}^{\prime}+\sec \theta_{v}^{\prime}-\bar{O}\left(\theta_{i}, \theta_{v}, \varphi\right)\right]} \quad$ where $\quad \bar{O}\left(\theta_{i}, \theta_{v}, \varphi,\right) . \quad$ The output represented the average of the overlap function $O\left(\theta_{i}, \theta_{v}, \varphi, h\right)$ between illumination and viewing shadows of the georeferenced riverine-based georeferenced $S$. damnosum s.l. larval habitat and its associated within-canopy shaded structures (e.g., Precambrian rock). Furthermore, $\phi$ was the difference in azimuth angle between viewing and illumination positions of the QuickBird imaged objects associated to the georeferenced riverine larval habitat. To simplify the equation, we approximated the overlap function by the overlap area and center positions of the ellipses. This approximation is justified when solar zenith and viewing zenith angles are not too large [21]. In the case of long ellipsoidal shadows, however, this approximation could have overestimated the width of the canopied $S$. damnosum s.l. riverine habitat hotspot in the azimuthal direction and underestimated the width of the hotspot in the azimuthal direction. To improve the accuracy and preserve the proper hotspot width information, we developed another approximation as follows. We used the equations $\phi=0$ or $\phi=\pi$. First, we considered the overlap function in the principal plane. We then used W $\phi=0$ or $\pi$ as the elliptical illumination and viewing shadows were aligned in the same direction. Thereaf ter, the overlap area was approximated by an ellipse with one axis equal to the overlap length and the other equal to the canopy shaded $S$. damnosum s.l. riverine larval habitat width encompassing the Precambrian rock and ripple water mixel spectral components which yielded

$$
O\left(\theta_{i}, \theta_{v}, \varphi\right)=\frac{1}{2}\left[\sec \theta_{i}^{\prime}+\sec \theta_{v}^{\prime}-\frac{h}{b}\left|\tan \theta_{i}^{\prime}-\tan \theta_{v}^{\prime} \cos \varphi\right|\right] .
$$

Spatial linear predictors were generated from the canopy, shaded, S. damnosum s.l., riverine, larval habitat and its associated Precambrian rock and rippled water components using an Ordinary kriged-based interpolator. The dependent variable was the explanatory spectral emissivity estimates QuickBird band ratio of the riverine larval habitat capture point rendered from the decomposition of the QuickBird pixel. We transformed the emissivities to fulfill the diagnostic normality test prior to performing kriging. Interpolation algorithms can generate accurate autoregressive endmember endemic transmission-oriented geopredictive risk maps [2].

In this research, the unknown value $Z\left(x_{0}\right)$ was interpreted as a random variable located in $\left(x_{0}\right)$, as well as the values of neighbor's

samples $\left(Z x_{i}\right), i=1, \ldots, N$. The estimator $\mathrm{Z}\left(\mathrm{x}_{0}\right)$ was also interpreted as a random variable located in the interpolator $x_{0}$, as result of the linear combination of the $S$. damnsoum s.l. spectral endmember predictor variables. In order to deduce the kriging system for the assumptions of the model, the following error committed while estimating $Z(x)$ was estimated using

$$
\in\left(x_{0}\right)=\hat{Z}\left(x_{0}\right)-Z\left(x_{0}\right)=\left[W^{T}-1\right] \cdot\left[Z\left(x_{i}\right) \ldots Z\left(x_{N}\right) Z\left(x_{0}\right)\right]^{T}=\sum_{i=1}^{N} w_{i}\left(x_{0}\right) \times Z\left(x_{i}\right)-Z\left(x_{0}\right)
$$


Citation: Jacob B, Novak RJ, Toe L, Sanfo MS, Caliskan S, et al. (2013) Unbiasing a Stochastic Endmember Interpolator Using ENVI Object-Based Classifiers and Boolean Statistics for Forecasting Canopied Simulium damnosum s.I. Larval Habitats in Burkina Faso. J Geophys Remote Sensing 2: 109. doi:10.4172/2169-0049.1000109

The quality criteria was then expressed in terms of the mean and variance of the new random variable $\in\left(x_{0}\right)$. Since the random function was stationary, [i.e., $E\left(Z\left(x_{i}\right)\right)=E\left(Z\left(x_{0}\right)\right)=m$ ], the following constraint was observed: $\quad E\left(\in\left(x_{0}\right)\right)=0 \Leftrightarrow \sum_{i=1}^{N} w_{i}\left(x_{0}\right) \times E\left(Z\left(x_{i}\right)\right)-E\left(Z\left(x_{0}\right)\right)=0 \Leftrightarrow$ $\Leftrightarrow m \sum_{i=1}^{N} w_{i}\left(x_{0}\right)-m=0 \Leftrightarrow \sum_{i=1}^{N} w_{i}\left(x_{0}\right)=1 \Leftrightarrow 1^{T} . W=1$. In order to ensure that a kriged model is unbiased, the sum of weights needs to be one [1].

The variance of the geopredictive endemic transmission-oriented model was then minimized employing $E\left(\in\left(x_{0}\right)^{2}\right)$. Two estimators can have $\in\left(x_{0}\right)=0$, but the dispersion around their mean determines the difference between the quality of estimators [1]. Our model rendered the following

$$
\begin{aligned}
\operatorname{Var}\left(\in\left(x_{0}\right)\right) & =\operatorname{Var}\left(\left[W^{T}-1\right] \cdot\left[Z\left(x_{i}\right) \ldots Z\left(x_{N}\right) Z\left(x_{0}\right)\right]^{T}\right)= \\
& \left.* W^{T}-1\right] \cdot \operatorname{Var}\left(\left[Z(x i) \ldots Z\left(x_{N}\right) Z\left(x_{0}\right)\right]^{T}\right) \cdot\left[\begin{array}{c}
W \\
-1
\end{array}\right]
\end{aligned}
$$

and

$$
\operatorname{Var}\left(\in\left(x_{0}\right)\right) \stackrel{*}{=}\left[W^{T}-1\right] \cdot\left[\begin{array}{cc}
\operatorname{Var}_{x i} & \operatorname{Cov}_{x_{i} x_{0}} \\
\operatorname{Cov}_{x_{i} x_{0}}^{T} & \operatorname{Var}_{x 0}
\end{array}\right] \cdot\left[\begin{array}{c}
W \\
-1
\end{array}\right]
$$

where the literals $\left\{\operatorname{Var}_{x_{i}}, \operatorname{Var}_{x_{0}}, \operatorname{Cov}_{x_{i} x_{0}}\right\}$ represented

$$
\left\{\operatorname{Var}\left(\left[Z\left(x_{1}\right) \ldots Z\left(x_{N}\right)\right]^{T}\right), \operatorname{Var}\left(Z\left(x_{0}\right)\right), \operatorname{Cov}\left(\left[Z\left(x_{1}\right) \ldots Z\left(x_{N}\right)\right]^{T}, Z\left(x_{0}\right)\right)\right\} \text {. }
$$

We defined $\gamma(h)$ (i.e., variogram) in the endemic endmember transmission-oriented model in the analysis of $Z(x)$. We then wrote an expression for the estimation variance of the canopy, shaded, S. damnsoum s.l., larval habitat estimator using the function of the covariance between the samples and the covariances to estimate:

$$
\left\{\begin{array}{c}
\operatorname{Var}\left(\in\left(x_{0}\right)\right)=W^{T} \cdot \operatorname{Var}_{x_{i}} \cdot W-\operatorname{Cov}_{x_{i} x_{0}}^{T} \cdot W-W^{T} \cdot \operatorname{Cov}_{x_{i} x_{0}}+\operatorname{Var}_{x_{0}} \\
\operatorname{Var}\left(\in\left(x_{0}\right)\right)=\operatorname{Cov}(0)+\sum_{i} \sum_{j} w_{i} w_{j} \operatorname{Cov}\left(x_{i}, x_{j}\right)-2 \sum_{i} w_{i} C\left(x_{i}, x_{0}\right)
\end{array}\right.
$$

which minimized

$$
W^{T} \cdot \operatorname{Var}_{x_{i}} \cdot W-\operatorname{Cov}_{x_{i} x_{0}}^{T} \cdot W-W^{T} \cdot \operatorname{Cov}_{x_{i} x_{0}}+\operatorname{Var}_{x_{0}}
$$

For optimization purposes we used the following kriging system as in Jacob et al. [48]:

$$
\left[\begin{array}{c}
\hat{W} \\
\mu
\end{array}\right]=\left[\begin{array}{cc}
\operatorname{Var}_{x_{i}} & 1 \\
1^{T} & 0
\end{array}\right] \cdot\left[\begin{array}{c}
\operatorname{Cov}_{x_{i} x_{0}} \\
1
\end{array}\right]=\left(\begin{array}{cccc}
\gamma\left(x_{1}, x_{1}\right) & \cdots & \gamma\left(x_{1}, x_{n}\right) & 1 \\
\vdots & \ddots & & \vdots \\
\gamma\left(x_{n}, x_{1}\right) & \cdots & \gamma\left(x_{n}, x_{n}\right) & 1 \\
1 & \cdots & 1 & 0
\end{array}\right)^{-1}\left(\begin{array}{c}
\gamma\left(x_{1}, x^{*}\right) \\
\vdots \\
\gamma\left(x_{n}, x^{*}\right) \\
1
\end{array}\right)
$$

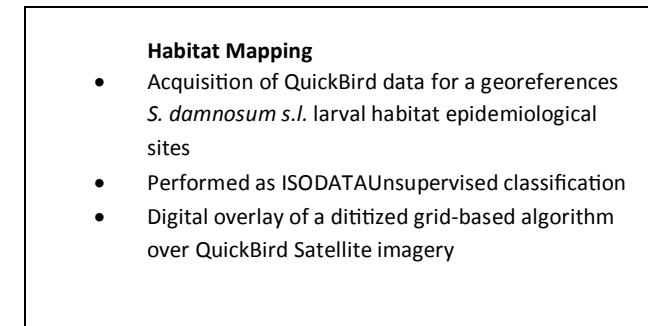

Successive Progressive Algorithm

- Spectrally Seperated with-in canopy geospatia feature attributes (e.g. Precambrian rock, ripple water components etc.) in $\mathrm{n}$-dimensional space

- Quantitation of sub-pixel endmember heterogeneity
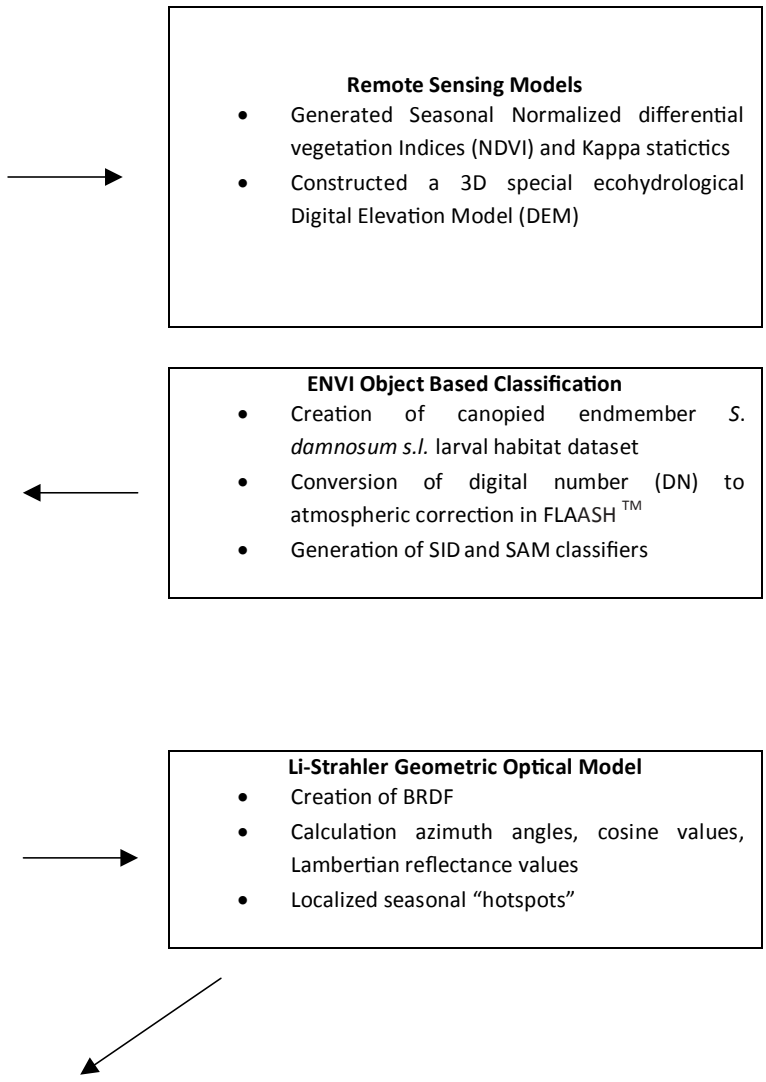

Validation

- Field Verification using "groundtruthing" exercises of all forecasted $S$. damnosum s.l. larval habitats

Scheme 1: Flow chart of spectral endmember interpolation a canopy shaded S. damnosum s.I. riverine larval habitat. 
Citation: Jacob B, Novak RJ, Toe L, Sanfo MS, Caliskan S, et al. (2013) Unbiasing a Stochastic Endmember Interpolator Using ENVI Object-Based Classifiers and Boolean Statistics for Forecasting Canopied Simulium damnosum s.I. Larval Habitats in Burkina Faso. J Geophys Remote Sensing 2: 109. doi:10.4172/2169-0049.1000109

Page 26 of 36

Where the additional parameter $\mu$ was a Lagrange multiplier employed in the minimization of the kriging error $\sigma_{k}^{2}(x)$ to honor the unbiasedness condition. Finally, the estimation and error by Ordinary kriging was given by:

$$
\hat{Z}\left(x_{0}\right)=W^{T} \cdot\left[Z\left(x_{1}\right) \ldots Z\left(x_{N}\right)\right]^{T} ; \operatorname{var}\left(\hat{Z}\left(x_{0}\right)-Z\left(x_{0}\right)\right)=\hat{W}^{T} \cdot\left[\gamma\left(x_{1}, x_{0}\right) \ldots \gamma\left(x_{N}, x_{0}\right)\right]^{T} .
$$

We ground truthed all residual forecasts from the stochastic interpolator. A flow chart is provided summarizing all the algorithms and software used for the interpolation of a canopy, shaded, georeferenced, S. damnosum s.l., riverine, larval habitat QuickBird band ratio (Scheme 1).

Additionally, we tested our model residual forecast with that of current unmixing algorithms commonly applied for canopy-oriented endmember signature generation. This unmixing algorithm was based was on the following linear mixing model:

$$
\begin{aligned}
& R_{k}=\sum_{i}^{n} a_{i} \cdot E_{i, k}+\varepsilon_{k} \quad R M S E=\sqrt{\left(\sum_{k}^{m} \varepsilon_{k}^{2}\right)^{-m}} \\
& R_{k} \quad \text { Reflectance of source at wavelength } k \\
& E_{k, i} \quad \text { Reflectance of endmember } i \text { at wavelength } k \\
& a_{i} \quad \text { Abundance of endmember } i \\
& a_{k} \quad \text { Error at wavelength } k \\
& R M S E \text { Root Mean square error of the } \varepsilon_{k} \\
& n \quad \text { Number of endmembers } \\
& m \text { Number of wavelengths in the discrete spectrum }
\end{aligned}
$$

This model assumed that spectrum is a linear superposition of the S. damnosum s.l. shaded canopy-related endmembers. The abundances were computed using two standards the algorithm assumed that wavelengths were given in nanometers (i.e., BEAM's default wavelength unit). The spectral unmixing tool was invoked from VISAT's tool menu by selecting the Unmix command and by using the command line tool gpt (Graph Processing Tool) found in BEAM's bin directory in batch mode (Figure 10, Figure 11, Figure 12). The differential form of the equation for the standard spectral unmixing model was:

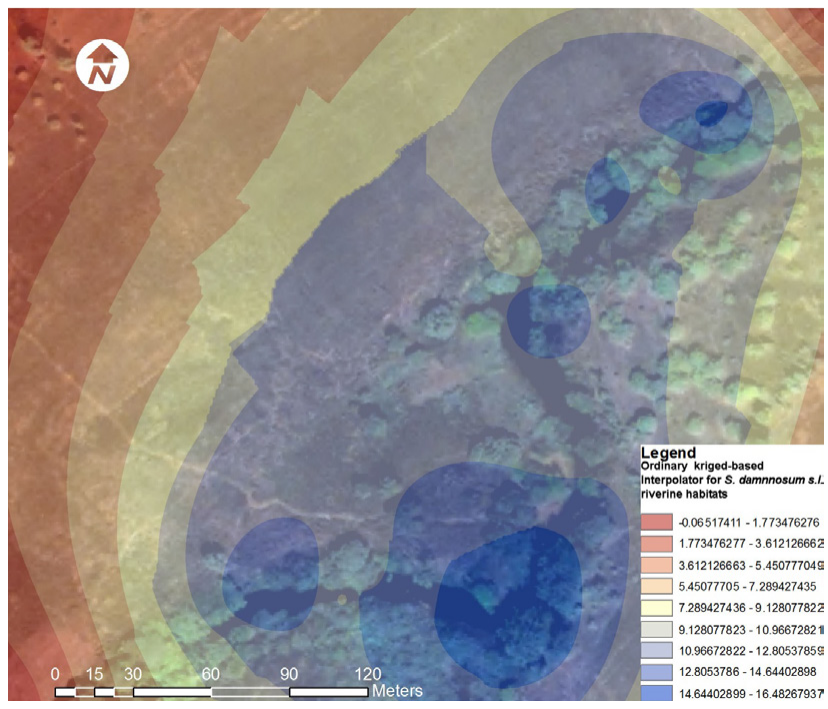

Figure 10: Kriged canopy shaded S.damnosum s.l. habitat pixel spectral reflectance estimates with predicted spectroradiometric measurements using a QuickBird endmember reference signature.

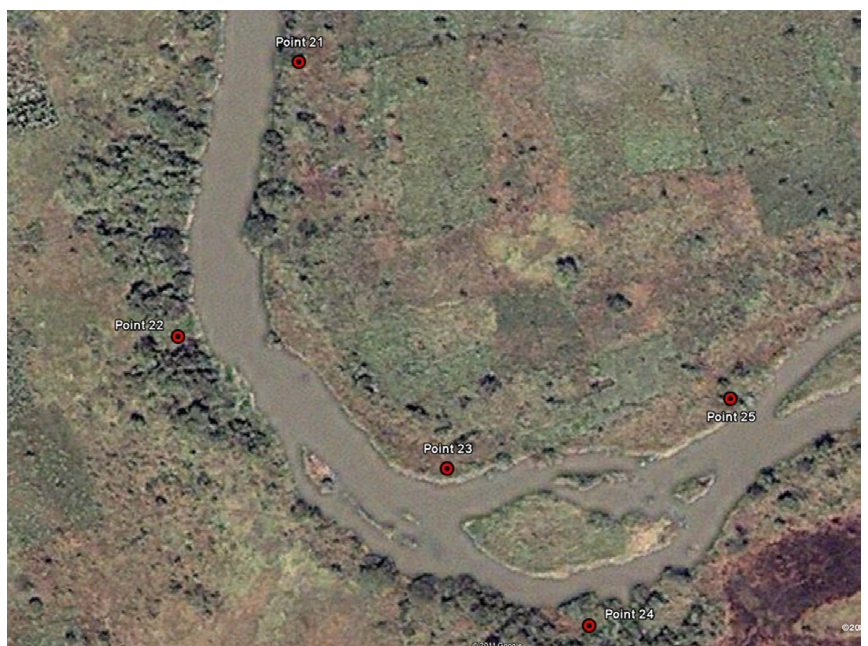

Figure 11: Predicted canopy shaded S.damnosum s.l. habitats ecosystem at the Dienkoa study site.

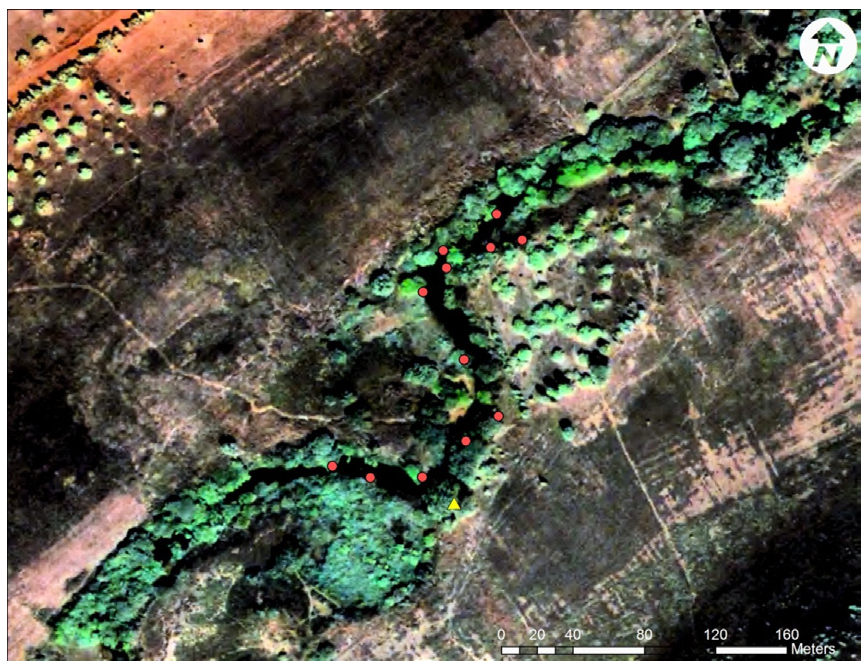

Figure 12: Predicted canopy shaded S. damnosum s.l. riverine larval habitats in a neighbouring riverine ecosystem in Burkina Faso.

$$
\frac{1}{c} \frac{\partial}{\partial t} I_{v}+\hat{\Omega} \cdot \nabla I_{v}+\left(k_{v, s}+k_{v, a}\right) I_{v}=j_{v}+\frac{1}{4 \pi c} k_{v, s} \int_{\Omega} I_{v} d \Omega
$$

where $j_{v}$ was the georeferenced, canopy, shaded, S. damnosum s.l. riverine, larval habitat emission coefficient, $k, v s$ was the scattering cross section, and $k_{v, a}$ was the absorption cross section. We noticed that in our model the differences were essentially due to the various forms for the canopy-related emission and absorption coefficients.

The emission coefficient and absorption coefficient are functions of temperature and density are related by: $\frac{j_{v}}{\dot{a}_{v}}=B_{v}(T)$ where is the black body spectral radiance at temperature $T$ [1]. The solution to the equation of our predictive radiative transfer was then: $I_{v}(s)=I_{v}\left(s_{0}\right) e^{-\tau_{v}\left(s_{0}, s\right)}+\int_{s_{0}}^{s} B_{v}\left(T\left(s^{\prime}\right) e^{-\tau_{v}\left(s^{\prime}, s\right)} d s^{\prime}\right.$

We employed the Eddington approximation for inserting 
our seasonal-sampled remote explanatory covariates. The Eddington approximation is a special case of the two stream approximation commonly applied in spectral unmixing algorithms [1]. The approximation assumed that the intensity in the georefernced, canopied, S. damnosum s.l., riverine, larval habitat was a linear function of $\mu=\cos \theta$. (i.e. $I_{v}(\mu, z)=a(z)+\mu b(z)$ where $z$ was the normal direction to the medium. Note that expressing angular integrals in terms of $\mu$ simplified inserting the sampled covariates as $d \mu=-\sin \theta \mathrm{d} \theta$ which appeared in the Jacobian of integrals as spherical coordinates.

Spatial linear predictors were generated from the canopy, shaded, S. damnosum s.l., larval habitat, and the Precambrian rock and riffled water components using an ordinary kriged-based interpolator and traditional Landsat data. The dependent variable was the spectral emissivity estimates generated from the decomposition of the QuickBird pixel which was transformed to fulfill the diagnostic normality test prior to performing kriging. The Ordinary kriged-based algorithm was then used to generate predictive, autoregressive, risk maps. Ordinary kriging was selected to interpolate the value $Z\left(x_{0}\right)$, the $S$. damnosum s.l. larval habitat and its associated Precambrian rock and riffle water components, $Z(x)$, at an unobserved habitat location $\mathrm{x}_{0}$ from the field and remote-sampled covariate coefficient estimates and $\mathrm{z}_{\mathrm{i}}=Z\left(x_{i}\right), \mathrm{i}=1 \ldots, \mathrm{n}$ at nearby predicted riverine habitat locations, $x_{1}, x_{n}$. Ordinary kriging is computed as a linear unbiased estimator, $\left(x_{0}\right)$ of $Z\left(x_{0}\right)$, based on a stochastic model of the dependence quantified by the variogram $(x, y)$ and by the expectation $(x)=E[Z(x)]$ and the covariance function $c(x, y)$ of the random field [2]. The kriging estimator was given by a linear combination of the algorithm: using the canopy, shadedrelated, endmember, spectral dataset of $\mathrm{z}_{\mathrm{i}}=Z\left(x_{i}\right)$ with weights $\mathrm{w}_{\mathrm{i}}\left(\mathrm{x}_{\mathrm{o}}\right)$, $\mathrm{i}=1, \ldots, \mathrm{n}$ chosen such that the variance in the spectral autoregressive predictive model was calculated using which was further minimized similar to the interpolation in the model using multiple unmixing algorithms and the ENVI classifers.

\section{Results}

Initially, we modeled the canopy, shaded, riverine, larval habitat study site areas using QuickBird-derived NDVI parameters. The QuickBird NDVI model reduced the heterogeneous spectralradiometric characteristics within the study site LULC while normalizing potential atmospheric effects. Invariant effects (e.g., ozone absorption and molecular scattering) and variant effects (e.g., water vapour absorption and aerosol scattering) of surface reflectance can generate uncertainties in atmospheric effects for NDVI modelling vector arthropod-related habitats [23]. Our assumption was that the physical basis of the differences in QuickBird NDVI parameters at the sample site may have been mainly attributable to the absorption of incident red light by plant chlorophyll and scattering of incident NIR radiation of the georeferenced habitat. Chlorophylls, the primary photosynthetic pigments in green plants, absorbs light primarily from the red and blue portion of the spectrum, while a higher portion of IR is reflected or scattered as a result of variously growing health vegetation which typically has low red light reflectance and high NIR reflectance [25]. Leaf cells have also evolved to scatter (i.e., reflect and transmit) solar radiation in the NIR spectral region [20]. This variation in reflectance and transmittance may have originated in the canopy, shaded, georeferenced, S. damnosum s.l., riverine, larval habitat from multiple reflections within the internal floating leaf structures causing different refractive indices of the leaf cell wall based on intracellular activities. The characteristics of light reflectance and transmittance can be explained mainly on the basis of critical reflection of visible light at the cell-wall-air interface of both the palisade and spongy layers of the mesophyll [28-34].
QuickBird NDVI parameter estimators were then generated in ArcGIS. For each NDVI value, the total areas were determined for specific surface vegetation-cover classes associated to the sampled, canopy, shaded, S. damnosum s.l. habitat (e.g., floating and emergent vegetation), and for a hybrid vegetation-cover class consisting of open water and submersed vegetation surrounding the habitat. The vegetation-oriented LULC-cover class comprising the largest total area was then assigned to NDVI value. The equation ArcGIS Image Server used to generate the output (i.e., NDVI $=$ arctangent $((\mathrm{IR}-\mathrm{R}) /(\mathrm{IR}+\mathrm{R}))$ produced a single-band dataset. The differential reflection in the QuickBird red and IR bands enabled quantitating density and intensity of green vegetation growth using the spectral reflectivity of solar radiation. For example, green leaves surrounding the canopied S. damnosum s.l. riverine larval habitat showed better reflection in the NIR wavelength range than in visible wavelength ranges. When leaves are water stressed, diseased, or dead, they become more yellow and reflect significantly less in the NIR [1]. The final index outputs values ranged between -1.0 and 1.0. Very low values of NDVI ( 0.1 and below) corresponded to the Precambrian rock, moderate values represented shrub surrounding the habitat ( 0.2 to 0.3 ), while high values indicated floating leaves and emergent vegetation within the canopy (0.6 to 0.9). The emissivities of most floating leaves surrounding the sampled $S$. damnosum s.l. riverine habitat were between about 93 to 98 .

We generated a correlation error matrix to determine the accuracy of the parameters. The row in the matrix represented the NDVI maps constructed from the QuickBird visible and NIR data, while the columns represented the reference data (ground truth, in-situ sampled data). We generated measures of thematic accuracy including overall classification accuracy and percentage of omission. In this research, the percentage of omission was the percentage of QuickBird pixels that were in a given NDVI class but, were not identified. Additionally, we generated the commission error which indicated pixels that were not identified but, were within a particular NDVI class. Predicted sizes of open-water, submersed and floating-and-emergent cover areas were closely inspected for observed sizes in the spatiotemporal-sampled dataset of the riverine ,larval habitat, field observations and corresponding QuickBird images. A residual normalized weighted matrix output was then generated using the users and producers accuracy-combined measures and the field-verified estimates of the QuickBird NDVI thematic canopied S. damnsoum s.l. riverine larval habitat mapping explanatory predictor variables. As primary accuracy measures, these relative entropy change measures were normalized by the arithmetic mean of the entropies generated by the proxy map variables. The overall classification accuracy of the QuickBird NDVI thematic maps revealed about $93 \%$ with a kappa value of 89 . The producer's accuracy ranged from 91.78 (canopyoriented, riverine, habitat attributes) to 92.64 (LULC surrounding the georeferenced habitats), while the user's accuracy was 92.37 and 93.74, respectively. The spatial variation of the QuickBird NDVI parameters were measured for determining surfaces of the georeferenced, canopied, S. damnosum s.l., riverine, larval habitats within the study site. It was found that emissivity was highly correlated with QuickBird NDVI after logarithmic transformation, with a correlation coefficient of $\mathrm{R}=0.93$.

We constructed 3-D QuickBird DEM to generate the spatial ecohydrological model employing the DGPS coordinates of the spatiotemporal-sampled, S. damnosum s.l., riverine, larval habitats inside OrthoEngine ${ }^{\circledR}$ software. Initially, a RPC block bundle model was computed. A pair of quasi-epipolor images was generated from the stereo images to retain elevation parallax in the X direction. An automated image-matching procedure was then used to produce the 
DEM through a comparison of the respective gray values. To find the corresponding pixels in the left and right quasi-epipolar images, we used a hierarchical sub-pixelmean cross-correlation match technique. The actual matching method generated correlation coefficients between 0 and 1 for each matched QuickBird canopied S. damnosum s.l. habitat pixel where 0 represented a total mismatch and 1 represented a perfect match. A second order surface was fitted around the maximum correlation coefficients. The difference between the georeferenced, canopied, S. damnosum s.l., riverine, larval habitats in the image provided the disparity (i.e., parallax arising from the terrain relief). This data was then converted to absolute elevation values above the WGS-84 ellipsoid using a 3-D space intersection solution. The data were then analyzed in a Pearson correlation matrix which revealed that the geomorphologic, explanatory, covariate slope was associated with the canopy, shaded, riverine, larval habitat. In this research to validate the slope-dependent covariate coefficients, we used the expression: St $=$ degrees $\left(\tan ^{-1}(S)\right)=360 \tan ^{-1}(S) / 2 \pi$. Thereafter, an analytical surface, $F(x, y)$ based on the parameter slope was defined as the magnitude of the first derivative of the sampled surface function. Our model assumed that a plane surface can be placed at any georeferenced, canopied, S. damnosum s.l., riverine, larval habitat point on the study site surface, $F(x, y)$ based on the parameter slope was defined as the magnitude of the first derivative of the sampled surface function. Our model assumed that a plane surface can be placed at any georeferenced, canopied, S. damnosum s.l., riverine, larval habitatpoint on the study site surface [(i.e. $F(x, y)$ ] generating a tangential plane which, in our model was based on the Euclidean-distance between the sampled habitats that was calculated using

$$
s=\sqrt{\left(\frac{\partial F}{\partial \mathrm{x}}\right)^{2}+\left(\frac{\partial F}{\partial \mathrm{y}}\right)^{2}} \text {. We used an angle } \boldsymbol{\alpha} \text { to adjust the }
$$

gradient vector in the QuickBird 3-D DEM. Th s enabled the submeter resolution slope-oriented values for the georeferenced larval habitats to be computed in any path direction (i.e., directional derivatives). If the differential components for gradient and curvature have been calculated for a surface, then directional derivatives can be computed with very little further computation [2]. Using the QuickBird 3-D DEM model we also generated catchment-related geomorphological, terrain-related statistics associated with riverine larval habitat surface values which in this research was represented by the spatial ecological empirical dataset of elevation-based covariate coefficients employing xyz coordinates which were validated using

$$
s=\sqrt{\left(\frac{z_{\varepsilon}-z_{\mathrm{w}}}{2 \Delta \mathrm{x}}\right)^{2}+\left(\frac{z_{N}-z_{S}}{2 \Delta \mathrm{y}}\right)^{2}} .
$$

The Pearsons matrix revealed that there was no correlation with elevation and the geosampled riverine larval habitats.

The spatial ecohydrological model generated from the canopy, shaded, S. damnosum s.l., riverine, larval habitat, geomorphologicalrelated, explanatory, predictor, covariate coefficient estimates revealed that the parameter slope was an important predictor variable in the estimator dataset. Altitudinal gradient is a strong determinant of the presence of species composition and abundance of black-fly vectors which also differ in vectoral competence and capacity [3]. In this research, slope gradient was defined as the maximum rate of change in altitude $(\tan \Theta)$, aspect $(\psi)$ as the compass direction of this maximum rate of change at a sampled, canopy, shaded, S. damnosum s.l., riverine, larval habitat. Slope, is commonly modeled by the DEM at a point and then classified as a vector; as such it has a quantity (i.e., gradient) and a directional aspect defined by a plane tangent to a topographic surface [26]. More analytically, in this research, slope gradient at a spatiotemporal-sampled, S. damnosum s.l., riverine, larval habitat was the first derivative of elevation $(\mathrm{Z})$ with respect of the slope $(S)$, where $S$ was in the aspect direction $(\psi)$. At the same time, the first derivative of a function at a spatiotemporal-sampled habitat was defined as the angular coefficient of the tangent to the function of a sampled habitat, hence: $\tan \Theta=$ rise/run $=\partial Z / \partial S$. The tangent function provided the slope $y$-component divided by the $x$ component in the QuickBird 3-D DEM. From slope, flow direction was determined; from flow direction, the upslope area that contributed flow to a sampled, riverine, canopied habitat within a QuickBird digitized grid cell was calculated. Upslope contributing area was then used to identify ridges and valleys in the riverine, epidemological study site. The upslope area (A) and slope (TanB) grids were used to obtain a topographic index (i.e., natural logarithm of the upslope contributing area per unit contour length divided by slope), expressed as $\ln (\mathrm{A} / \tan \mathrm{B})$. In our QuickBird 3-D DEM and TINs provided canopy surface values at every spatiotemporalsampled, canopy, shaded, S. damnosum s.l., riverine, larval habitat point using a non-linear function. Typically, surfaces within GIS are rarely represented by analytic functions as they are usually modeled as TINs or, grids with a finite resolution [20].

We generated pixel-based spectral metrics from the remotelysampled canopied S. damnosum s.l. riverine larval habitat and its associated Precambrian rock and ripple water constituents using an object-based classified. In this research, ENVI ${ }^{\circledR}$ software was used to automatically categorize individual pixels of the canopy, shaded, $S$. damnosum s.l., habitats and their associated neighboring Precambrian rock and ripple water components into separate spectral classes. Then we converted the remotely-sensed raster layers to vector coverage's which were then classified as ESRI shapefiles. The object-based classifier provided interactive spatial/spectral pixel editing for the Precambrian rock and ripple water image components. The ENVI ${ }^{\circledR}$ Feature Extraction module then automated the process of performing accurate segmentations. For example, the FLAASH model included a method for retrieving selected "dark" pixels of the Precambrian rock in the QuickBird scene. The spectral profile based on the image endmembers were then extracted using the SAM. The algorithm determined the spectral similarity between the extracted canopy, shaded, $S$. damnosum s.l., radiance by calculating the angle between the emissivity estimates and by treating the reflectance spectra as vectors in a space with dimensionality equal to the number of QuickBird bands. The FLAASH model output and SAM can compare the angle between the endmember spectrum vector and the sampled pixel spectrum vector in $n-D$ space (www.itvis.com). The SAM calculated the spectral similarity between the QuickBird, canopy, shaded, S. damnosum s.l. habitat image spectrum and the reference, canopy, reflective spectra which revealed that the data was not affected by solar illumination factors as the angle between the vectors was independent of the vectors length. Smaller angles between the endmember spectra represent closer matches to the reference spectrum in a spectral library [27]. QuickBird pixels further away than the specified maximum angle threshold in radians were not classified. In this research, we also used the Spectral Information Divergence (SID) classification. The SID compared the similarity between the selected QuickBird, canopy, shaded, S. damnosum s.l., riverine, larval habitat mixels and the Precambrian rock and ripple water components by measuring the probabilistic discrepancy between the corresponding spectral emissivities The SID successfully calculated all the spectral probabilities of the sampled, canopy, shaded, S. damnosum s.l., habitat data in the library related to the QuickBird pixels.

In this research, the spectral library in the object-based classifier accounted for all processes and factors influencing the reflectance spectral endmember data collection from the sampled, canopy, shaded, 
S. damnosum s.l., riverine, larval habitat and its associated Precambrian rock and ripple water components. After the calibration coefficients had been determined, the $S$. damnosum s.l. habitat image was converted to match the library-based data. Analogously, the reference endmembers spectra in the library were transformed into the endmembers spectra of the imaged habitat. In this research we expressed these pixels as linear combinations of image endmembers. A function incorporating the calibration and the alignment were repeatedly evaluated for different candidate groups of reference spectra emitted from the canopy, shaded, S. damnosum s.l., riverine, larval habitat until a suitable representation of the canopy endmembers was determined.

We then used an SPA to generate endmembers from the canopy, shaded, S. damnosum s.l. habitat, the Pre-Cambrian rock and the ripple water spectra. We let $P(i, j)$ denote the spectrum for the spectrally extracted QuickBird pixel using the image coordinates $(i, j)$, as the foundation of the unmixing algorithm which in this research was defined by using $\vec{p}_{(i, j)}=\sum_{k=1}^{m} f_{(i, j) k} \vec{e}_{k}+\vec{\varepsilon}_{(i, j)}$ and also $f_{(i, j) k} \geq 0, k=1, \ldots, m \sum_{k=1}^{m} f_{(i, j) k}=1$ where $m$ was the number of endmembers, was the $\mathrm{k}^{\text {th }}$ endmember $\bar{\varepsilon}_{(i, j)}$ was the approximation error term (i.e., residual), and $f(i, j)^{k}$, was the fractional abundance for the $k t h$ endmember of pixel $(i, j)$. We omitted the error term in $\vec{p}_{(i, j)}=\sum_{k=1}^{m} f_{(i, j) k} \vec{e}_{k}+\vec{\varepsilon}_{(i, j)}$ and computed the possible linear mixtures from $\vec{p}_{(i, j)}=\sum_{k=1}^{m} f_{(i, j) k} \vec{e}_{k}+\vec{\varepsilon}_{(i, j)}$ and $f_{(i, j) k} \geq 0, k=1, \ldots, m \sum_{k=1}^{m} f_{(i, j) k}=1$ which formed a simplex $\mathrm{Cm}$ defined by $m$ vertices that corresponded to the S. damnosum s.l. habitat endmembers, $\vec{e}_{1}, \vec{e}_{2}, \ldots, \vec{e}_{m}$. The volume of the simplex $\mathrm{Cm}$ was then calculated from the equation $V\left(C_{m}\right)=\frac{1}{(m-1) !}\left|\operatorname{det}\left(W W^{T}\right)\right|^{\frac{1}{2}}$ where $w=\left[\vec{e}_{2}-\vec{e}_{1}, \vec{e}_{3}-\vec{e}_{1}, \ldots, \vec{e}_{n}-\vec{e}_{1}\right]$ was the volume of the simplex defined

by $m$ canopied $S$. damnosum s.l. riverine larval habitat endmembers, and where det $(\cdot)$ denoted the determinant of a matrix representing the operation of an absolute value. In this research, once the endmembers $\vec{e}_{1}, \vec{e}_{2}, \ldots, \vec{e}_{m}$ were determined their abundance was estimated through the least squares method which was equivalent to a projection on the simplex.

In the geometrical-optical model the effect of sunlit canopy on the bidirectional reflectance was quantified using the second termin equation

$R(i, v)=K_{g} G+\frac{C}{A} \iint_{A_{c}} \frac{\langle i, s\rangle}{\cos \theta_{i}} \frac{\langle v, s\rangle}{\cos \theta_{v}} d s$. This variation depended on both the density and angular distribution of $d s$ in the equation. We assumed that each object in a scene could be modeled as a sphere without mutual illumination shading between ds elements. Then, the second term was approximated as: $K_{C} C=\frac{1}{2}(1+\langle i, v\rangle)\left(1-e^{-\lambda \pi R^{\prime 2} \sec \theta_{v}}\right) C$ as in Strahler and Jupp [50]. In this expression, the first term was the illuminated proportion of the habitat area based on a single sphere viewed and illuminated at position $i$. This was weighted by the second term which was the proportion of the area of spheres visible from zenith angle $\theta_{v}$. Since both terms varied smoothly between zero and one, this contribution to the riverine habitat hotspot was quite $\mathrm{f}$ lat. In the case of a spheroid, we simply then replaced, $\langle i, v\rangle$ by $\left\langle i^{\prime}, v^{\prime}\right\rangle$ where $\left\langle i^{\prime}, v^{\prime}\right\rangle=\cos \theta_{i}^{\prime} \cos \theta_{v}^{\prime}+\sin \theta_{i}^{\prime} \sin \theta_{v}^{\prime} \cos \phi$.
The first term in equation $K_{c} C=\frac{1}{2}(1+\langle i, v\rangle)\left(1-e^{-\lambda \pi R^{\prime 2} \sec \theta_{v}}\right) C$ ignored the problem of mutual shading of the S. damnosum s.l. riverine habitat canopy, the Precambrian rock and the ripple water components. To carry this out, we developed an approach that applied one-stage geometric optics to deal with the spatial relationship between the subpixel endmember reflectance spectra of the decomposed S. damnosum s.l. habitat surface, Precambrian rock and ripple water components that was mutually shaded in the illumination direction and the parts mutually shaded in the view direction. We then quantified mutual shadowing proportions generated from the $S$. damnosum s.l. habitat and its associated Precambrian rock and riffle water components as in Li and Strahler [52].

The bidirectional reflectance was modeled as a pure phenomenon that resulted is scenes of discrete, three-dimensional objects (i.e., Precambrian rock and riffle water components rendered) from the sampled, canopy, shaded, S. damnosum s.l., riverine, habitat being illuminated and viewed from different positions in the hemisphere. The resulting scene was broken down into their canopy fractions specifically sunlit and shadowed background and scene brightness. Illumination direction was calculated by a linear combination of the canopy fractions and their respective radiance estimates. The shape of the Precambrian rock and patterns of the diffuse riffled water components were the driving explanatory spectral predictor variables in the model. These S. damnosum s.l. riverine habitats spectral sub-pixel emissivities conditioned the mixture of sunlit and shaded objects and background data that was observed from multiple viewing directions thus, quantifying all directions of illumination. This mixture, in turn, controlled, the brightness in the image. However, since the use of data for quantitative spectral monitoring requires consistent surface reflectance data (see 19) we corrected the radiance effects from varying sun sensor target geometries in the multitemporal QuickBird datasets described by the BRDF. After correction, the root mean square reflectance errors were approximately 0.01 in the Visible and 0.02 in the NIR.

In the geometric-optical model, the shape of the hotspot function was based on the viewing and illumination positions in the model, and these diverged due to the shape and height of the spheroids. The "hotspot" was defined as a site with a combination of Precambrian rock and turbid water sub-pixel endmember data associated with $S$. damnosum s.l. larval habitat. The equation $\mathrm{S}=(\mathrm{AgG}+\mathrm{ACC}+\mathrm{AZZ}+\mathrm{AtT})$ IA, where $S$ represented the reflectance of the QuickBird pixel; G, C, $\mathrm{Z}$, and $\mathrm{T}$ were the reflectance of sunlit ground and shadowed ground and canopy, respectively; Ag through A were the corresponding areas of the four components; and A was the pixel. These calculations were helpful to understand how the physical shape of the S. damnosum s.l. riverine habitat, Precambrian rock and riffle water components governed the shape of the overlap functions. The exact overlap function on the principal cone was also obtained using the hybrid of the geometric optical model for capturing bidirectional reflectance values over the riverine larval habitat attributes.

In the model, the viewing zenith was the viewing direction, which had a different azimuth than the illumination position. Rather than computing the overlap of ellipses rendered from the canopy, shaded, S. damnosum s.l., larval habitat, the Precambrian rock and its riffle water spectral components at arbitrary inclinations and distances directly, we instead fit a linear function to the diminution of the overlaps generated from the model residuals using azimuth angles. We approximated the azimuthal cut off of the hotspot and linearly interpolated the model residuals. The residual output from the equation was then used to determine that: 
(1) the azimuthal width of hotspot effect was basically determined by a ratio) and, (2) the outward width of hotspot on the principal plane was determined by ratio; and, (3) the inward width was determined by both. The spectral signature rendered was found to be characteristic of the canopy, shaded, S. damnosum s.l., larval habitat. The composition of this signature was $34 \%$ red, $11 \%$ blue and $55 \%$ green. This signature corresponded to habitats consisting of fast flowing water over a base of Precambrian rock.

In the spectral examination of our reference signature, the total surface area of the georeferenced, canopy, shaded, S. damnosum s.l. habitat and its associated Precambrian rock and ripple water components were then divided proportionally according to the fractional abundances generated from the larval habitat surface reflectance material. This endmember classification allowed the canopied radiance estimates of the sampled habitat data to convey the actual proportions of spectral characteristics in the decomposed QuickBird pixel endmember data. In this sense, a linear relationship existed between the fractional abundance of the substances comprising the decomposed, S. damnosum s.l., larval habitat pixel and its associated Precambrian rock and the ripple water endmember components being imaged, and the spectrum of the overall habitat reflectance values. The classification revealed that the reflected spectral data was separated by significantly contrasted pixel spectra (e.g., black sub-pixel Precambrian rock reflectance and white ripple water sub-pixel within canopied constituents). The model residuals also revealed that the spectrally separated endmember references within the canopy, shaded, S. damnosum s.l., larval habitat may not have been excessively different (e.g., floating leaf and grass reflectance spectra). Further, the model residuals also revealed a real fraction of sunlit canopy, sunlit background and shadow-parameters related to the $S$. damnosum s.l. riverine larval habitat.

Independently determined residual, canopy-related, spectrally dependent estimates for each of shadowed or sunlit portion of the georeferenced, $S$. damnosum s.l., habitat and its spatially associated Precambrian rock and ripple water components were then calculated by the geometric-optical model using spectral bidirectional reflectance values. Our model was similar to a hybrid of geometric optical and radiative transfer model used commonly for spectrally quantitating bidirectional reflectance over discontinuous plant canopies. For example, in our spatiotemporal BRDF model, the coupling was conducted by relaxing the assumption of Lambertian background. Thus, we defined the model and its residuals into, a nonLambertian model.

The non-Lambertian was modeled by the canopied, spatiotemporal, S. damnosum s.l., BRDF model. The shadowing behaviour in a canopy-oriented, S. damnosum s.l., riverine, larval habitat scene and its associated LULC features was also spectrally accommodated in the model through quantification of the enhanced single scattering (i.e., shadowing of the habitat spatial attributes). The model then rendered estimators, such as vegetation canopy as an assemblage of partially illuminated spatial objects using geometric optics and Boolean set theory which also subsequently deduced the proportion of shadowed, canopy in the decomposed, QuickBird, $S$. damnosum s.l., riverine, habitat sub-mixel explanatory covariate coefficients and its associated LULC feature data attributes as a function of view and illumination angles. In turn, the seasonally quantitated illumination angles determined the primary path that the incident light traced into the S. damnosum s.l. riverine habitat canopy cover and quantified the Precambrian rock and ripple water pixel emissivities illuminated. Whereas the path radiance and the upward transmittance depend on the thickness of the atmosphere between the surface and the sensor, specific spectral components can be modified by the relative orientation of the target pixel [1]. Directional reflctance values from the geometric-optical model was then integrated to generate hemispherical reflectance estimates for illumination angles using Boolean algorithms and the target spectral signature generated from the canopy, shaded, $S$. damnosum s.l. habitat and its associated Precambrian rock and ripple water components. The endmember extraction algorithms determined the shade-related factors that contributed to producing the DN of the sampled, canopy, shaded, $S$. damnosum s.l., larval habitat and its associated Precambrian rock and ripple water reflectance pixel which corresponded to an integration of geometric and radiometric parameters of the overall calculated radiance.

The influence of the local topography based on the tabulated BRDF emitted from the georeferenced S. damnosum s.l. habitat, however, had to be corrected prior to sub-pixel spectral land surface reflectance quantitation and albedo estimation. Reflectance standardization to a field view and sun angle should be performed using the pre-derived BRDF parameter estimators and proportional BRDF correction [20]. In order to avoid generating erroneous estimates in the interpolation algorithm, in this research, the direct and the diffuse irradiance of the canopy, shaded, S. damnosum s.l., larval habitat and its associated Precambrian rock and ripple water components were further spectrally evaluated using the angle between the habitat surface and the sun ray directions for determining the direct irradiant estimate. This estimate was computed by means of the cosine law from the georeferenced, spatiotemporal-sampled, terrain-related, spectrallydependent, explanatory predictor, covariates, coefficient estimates derived from the QuickBird 3-D DEM. Reflectance data can be corrected for BRDF effects by normalization to a standard viewing geometry [1]. A mask of cast shadows was also computed to isolate QuickBird, canopy, shaded, S. damnosum s.l., larval habitat spectral estimates which were not getting any direct irradiance. To estimate the irradiance, a terrain view factor was also computed in an analogous fashion. Finally, the DN's in the image were converted to scene radiances by means of the calibration coefficient estimates provided by the $S$. damnosum s.l. riverine larval habitat and its Precambrian rock and ripple water pixel reflectance emissivities. For each of this significant non-Lambertian reflectance behavior was observed, but regardless BRDF correction using the derived parameter estimators successfully minimized spectral variation in the radiance estimates. Thus, a set of distinct spectra that constituted the mixed, canopy, shaded, pixel spectra was identified in the endmember-determination stage.

Semivariograms were generated in ArcGIS ${ }^{\infty}$ Spatial Analyst which modeled the structure of spectral variability in the decomposed, QuickBird, canopy, shaded, S. damnosum s.l., habitat pixel. The semiovariogram was useful for summarizing the spatial continuity of the decomposed habitat data. Optimal linear predictors were generated by incorporating the model of the covariance of the random function using a weighted moving average interpolation and the QuickBird, $S$. damnsoum s.l., larval habitat, endmember, reference signature. The model measured the local variation in the reference signature as a function of distance and direction. There was no need to estimate a first-order trend; instead, the mean was estimated from the reference signature. Local deviations from the global mean were accounted for by the covariance model residual output.

Ordinary kriging was selected to interpolate the value $Z\left(x_{0}\right)$, (i.e., the canopy, shaded, $S$. damnosum s.l., riverine, larval habitat and its associated Precambrian rock and ripple water components) using $Z(x)$ (i.e., sampled explanator covariate coefficients) an $x_{0}$ (i.e., unobserved 
habitat location), where $\mathrm{z}_{\mathrm{i}}=Z\left(\mathrm{x}_{\mathrm{i}}\right), \mathrm{i}=1 \ldots, n$ was the nearby riverine larval habitat locations, $\mathrm{x}_{1}, \mathrm{x}_{\mathrm{n}}$. In this research, Ordinary kriging was computed as a linear unbiased estimator, $\check{Z}\left(\mathrm{x}_{\mathrm{o}}\right)$ of $Z\left(x_{0}\right)$ based on a stochastic model of the dependence quantified by the variogram $\gamma(x, y)$ and by the expectation $\mu(x)=E[Z(x)]$ and the covariance function $c(x, y)$ of the random field. The kriging estimator was given by a linear combination of the algorithm: $\hat{Z}\left(x_{0}\right)=\sum_{i=1}^{n} w_{i}\left(x_{0}\right) Z\left(x_{i}\right)$ employing the QuickBird spectral endmember dataset of $\mathrm{z}_{\mathrm{i}}=Z\left(\mathrm{x}_{\mathrm{i}}\right)$ with weights $\mathrm{w}_{\mathrm{i}}\left(\mathrm{x}_{\mathrm{o}}\right), \mathrm{i}=1, \ldots, n$ chosen, such that the variance in the interpolator was calculated using: $\sigma_{k}^{2}\left(x_{0}\right):=\operatorname{Var}\left(\hat{Z}\left(x_{0} o\right)-Z(x)\right)=\sum_{i=1}^{n} \sum_{j=1}^{n} w_{i}\left(x_{0}\right) w_{j}\left(x_{0}\right) c\left(x_{i}, x_{j}\right)+\operatorname{Var}(Z(x))-2 \sum_{i=1}^{n} w_{i}\left(x_{0}\right) c\left(x_{i}, x_{0}\right)$ which was further minimized using:

$$
E[\hat{Z}(x)-Z(\mho)]=\sum w_{i}\left(x_{0}\right) \mu\left(x_{i}\right)-\mu(x)=0 .
$$

The weights of the Ordinary kriged-based algorithm were used to fulfill the unbiasedness condition in the interpolation of the canopy shaded S. damnosum s.l. habitat endmember signature using $\sum_{i=1}^{n} \lambda_{i}=1$ which was provided by the equation system:

$$
\left(\begin{array}{c}
\lambda_{1} \\
\vdots \\
\lambda_{n} \\
1
\end{array}\right)=\left(\begin{array}{cccc}
\gamma\left(x_{1}, x_{1}\right) & \cdots & \gamma\left(x_{1}, x_{n}\right) & 1 \\
\vdots & \ddots & & \vdots \\
\gamma\left(x_{n}, x_{1}\right) & \cdots & \gamma\left(x_{n}, x_{n}\right) & 1 \\
1 & \cdots & 1 & 0
\end{array}\right)^{-1}\left(\begin{array}{c}
\gamma\left(x_{1}, x^{*}\right) \\
\vdots \\
\gamma\left(x_{n}, x^{*}\right) \\
1
\end{array}\right)
$$

We considered the optimization problem using maximize $f(x, y)$ subject to $g(x, y)=c$. We introduced a new explanatory predictor variable $(\lambda)$ (i.e., Lagrange multiplier) which in this research was defined by $\Lambda(x, y, \lambda)=f(x, y)+\lambda \cdot(g(x, y)-c$. Lagrangian multipliers can be used to find the extrema of a multivariate function $f\left(x_{1}, x_{2}, \ldots, x_{n}\right)$ subject to the constraint $g\left(x_{1}, x_{2}, \ldots, x_{\mathrm{n}}\right)=0$, where $\mathrm{f}$ and $\mathrm{g}$ are functions with continuous first partial derivatives on the open set containing the curve $g\left(x_{1}, x_{2}, \ldots, x_{\mathrm{n}}\right)=0$, and $\nabla g \neq 0$ at any point on the curve where $\nabla$ is the gradient [22]. Commonly, the Lagrange multiplier is used in the minimization of the kriging error $\sigma_{k}^{2}(x)$ to honor the unbiasedness condition in predictive vector arthropod-related habitat risk-based distribution models $[1,46]$. Th $\lambda$ term was added. If $(x, y)$ is a maximum for the original constrained problem, then there exists a $\lambda$ such that $(x, y, \lambda)$ is a stationary point for the Lagrange function [13]. In predictive autoregressive vector insect data analyses, georeferenced habitat points can determine optimal covariates when the partial derivatives of $\Lambda$ are zero [2]. The additional parameter $\mu$ was a Lagrange multiplier used in the minimization of the kriging error, $\sigma_{k}^{2}(x)$, to maintain the unbiasedness condition in the endmember dataset. In mathematical optimization, the method of Lagrange multipliers provides a strategy for finding the maxima and minima of a function subject to constraints [13].

We considered the optimization problem using maximize $f(x, y)$ subject to $g(x, y)=$ c. Throughout the interpolation effort, the independent spectrally-dependent explanatory predictor variables were denoted as $\mathrm{p}=\left(x_{1}, x_{2}, \ldots, x_{\mathrm{N}}\right)$, while the function being analyzed was denoted by $\mathrm{f}(\mathrm{p})$ and the constraints were represented by the equations $g_{1}(p)=0, g_{2}(p)=0, \ldots, g_{M}(p)=0$. We introduced a new variable $(\lambda)$ (i.e., Lagrange multiplier), and the Lagrange function:

$$
\Lambda(x, y, \lambda)=f(x, y)+\lambda \cdot(\bar{g}(x, y)-\bar{c}) .
$$

Our strategy was to use the Lagrange multipliers to accommodate constraints in the autoregressive canopy shaded S. damnosum s.l. habitat riverine larval habitat model. We considered a paraboloid with a constraint that was a single point, such that we had two line constraints that intersected. The level set (i.e., contour line) clearly appeared to "cross" that point and its gradient which was clearly not parallel to the gradients of either of the two line constraints. Yet, it was obviously a maximum and a minimum because there was only one point on the paraboloid that met the constraint. In this research, the hyperbolic paraboloid was parameterized simply as $\vec{\sigma}(u, v)=\left(u, v, \frac{u^{2}}{a^{2}}-\frac{v^{2}}{b^{2}}\right)$, which had a Gaussian curvature

$$
\begin{gathered}
K(u, v)=\frac{-4}{a^{2} b^{2}\left(1+\frac{4 u^{2}}{a^{4}}+\frac{4 v^{2}}{b^{4}}\right)^{2}} \text { and mean curvature of } \\
H(u, v)=\frac{-a^{2}+b 2-\frac{4 u^{2}}{a^{2}}+\frac{4 v^{2}}{b^{2}}}{a^{2} b^{2}\left(1+\frac{4 u^{2}}{a^{4}}+\frac{4 v^{2}}{b^{4}}\right)^{3 / 2}}
\end{gathered}
$$

In the construction of the spatial interpolation matrix our basic assumption was that if we considered only the georeferenced riverinebased habitat points that satisfied the canopy endmember constraints then a canopy shaded georeferenced habitat point with its associated within canopy data feature attributes $(p, f(p))$ was a stationary point of $f$ if, the constraints at that habitat point did not allow movement in a direction where $f$ changed value. A point on $f$ is a constrained stationary point if, and only if, the direction that changes violates at least one of the constraints [13]. We started by considering the level set of $f$ at $(p, f(p))$. The set of vectors $\left\{v_{L}\right\}$ contained the directions in which we moved and still remained in the same level set and where the value of $f$ did not change (i.e. the change equals zero). In this research, for every vector $v$ in $\{v\}$, the following equation was used $\Delta f=\frac{d f}{d x_{1}} v_{x_{1}}+\frac{d f}{d x_{2}} v_{x_{2}}+\ldots+\frac{d f}{d x_{N}} v_{x_{N}}=0$ where the notation $v_{x K}$ above meant that the $x_{K}$-component was derived from the vector $v$. The equation was then rewritten in a more compact geometric form as:

$$
\underbrace{\left[\begin{array}{c}
\frac{d f}{d x 1} \\
\frac{d f}{d x_{2}} \\
\vdots \\
\frac{d f}{d x_{N}}
\end{array}\right]}_{\nabla f} \cdot \underbrace{\left[\begin{array}{c} 
\\
v_{x 1} \\
v_{x 2} \\
v_{x N}
\end{array}\right]}_{\mathrm{v}}=0 \Rightarrow \nabla f \cdot \mathrm{v}=0
$$

The residual interpolation canopied endmember predictive autoregressive model output revealed that at $p$, all directions from the point did not change the value of $f$ which was perpendicular to $\nabla f(p)$ (i.e., the gradient of $f$ at $p$ ). The output also revealed that, for every vector $\mathrm{v}$ in $\left\{v_{c}\right\}$, the following held:

$$
\Delta g=\frac{d g}{d x_{1}} v_{x_{1}}+\frac{d g}{d x_{2}} v_{x_{2}}+\ldots+\frac{d g}{d x_{N}} v_{x_{N}}=0 \Rightarrow \nabla g \bullet v=0
$$

From this equation, we determined that at point $p$, all directions from this point still satisfied this constraint which was perpendicular to $\nabla g(p)$. Once the Lagrange parameters were generated they were introduced into the Ordinary kriged-based interpolator, which was 
expressed as:

$$
\hat{Z}\left(x^{*}\right)=\left(\begin{array}{c}
z_{1} \\
\vdots \\
z_{n} \\
0
\end{array}\right)^{\prime}\left(\begin{array}{cccc}
\gamma\left(x_{1}, x_{1}\right) & \cdots & \gamma\left(x_{1}, x_{n}\right) & 1 \\
\vdots & \ddots & \vdots & \vdots \\
\gamma\left(x_{n}, x_{1}\right) & \cdots & \gamma\left(x_{n}, x_{n}\right) & 1 \\
0 & \cdots & 1 & 0
\end{array}\right)^{-1}\left(\begin{array}{c}
\gamma\left(x_{1}, x^{*}\right) \\
\vdots \\
\gamma\left(x_{n}, x^{*}\right) \\
1
\end{array}\right)
$$

In the spatial analyst extension of ArcGIS $10.1^{\circledR}$, we calculated the new residual error variance, (i.e., interpolation variance) and output it along with the traditional kriging variance. The $95 \%$ error bound based on the new variance was reported also. The spatial autocorrelation error matrix was then provided by:

$$
\operatorname{var}\left(\hat{Z}\left(x^{*}\right)-Z\left(x^{*}\right)\right)=\left(\begin{array}{c}
\gamma\left(x_{1}, x^{*}\right) \\
\vdots \\
\gamma\left(x_{n}, x^{*}\right) \\
1
\end{array}\right)\left(\begin{array}{cccc}
\gamma\left(x_{1}, x_{1}\right) & \cdots & \gamma\left(x_{1}, x_{n}\right) & 1 \\
\vdots & \ddots & \vdots & \vdots \\
\gamma\left(x_{n}, x_{1}\right) & \cdots & \gamma\left(x_{n}, x_{n}\right) & 1 \\
1 & \cdots & 1 & 0
\end{array}\right)^{-1}\left(\begin{array}{c}
\gamma\left(x_{1}, x^{*}\right) \\
\vdots \\
\gamma\left(x_{n}, x^{*}\right) \\
1
\end{array}\right)
$$

Having created a covariance function with which to form the kriging weights matrix, the next task was to tessellate the region into patches.

In this research, the basic equation used in kriging of the canopy shaded S. damnosum s.l. endmember reference signature was as follows: $F(x, y)=\sum_{i=1}^{n} w_{i} f_{i}$ where $n$ was the number of scatter points in the spatiotemporal-sampled dataset, $\mathrm{f}_{\mathrm{i}}$ was the values of the scatter points, and wi was the spectral weights assigned to each scatter point as in Cressie (1993). In three dimensional space, $x$ our model represented the coordinates $(\mathrm{xu}, \mathrm{xv}, \mathrm{xw})$ where $\mathrm{h}$ represented a vector of modulus $|\mathrm{h}|$ and direction $\alpha$. In a condensed form, $\gamma(\mathrm{h})$ then represented the set of black fly-related larval habitat-related variogram $\gamma(|\mathrm{h}|, \alpha)$ in an unbiased fashion for each direction $\alpha$. There was no reason in this investigation to expect that the canopy shaded S. damnosum s.l., riverine larval habitat endmember interpolation would exhibit the same behavior in every direction (i.e., that the habitat radiance would be isotropic). Anisotropy is the property of being directionally dependent which can be defined as a difference, when measured along different axes, in a material's physical or mechanical properties (e.g., refractive index).

Incorporating geometric anisotropy in our Kriging procedure was simply a matter of applying an affine transformation to the reflected distance measurements. An affine transformation keeps point distances in one direction unchanged and stretches distances in the direction perpendicular to it [1]. In order to determine levels of anisotropy we used is a rotation of the $\mathrm{x}$-axis to a position parallel to a presumed axis where $\left(\begin{array}{l}x^{x} \\ y^{x}\end{array}\right)=Q \cdot\left(\begin{array}{l}x \\ y\end{array}\right)$ and where $Q=\left(\begin{array}{cc}\cos \alpha & \sin \alpha \\ -\sin \alpha & \cos \alpha\end{array}\right)$. In the model specifications, $\alpha$ was the rotation angle. In the direction of this major axis it was supposed that a variogram model with range R1 was applicable. At right angles to this direction a variogram model with shorter range R2 was also supposed [see 1,48].

The second step for incorporating anisotropy into our model was the transformation of the ellipse into a circle, which, in this research was performed by:

$$
\left(\begin{array}{l}
\mathrm{x}^{\mathrm{xx}} \\
\mathrm{y}^{\mathrm{xx}}
\end{array}\right)=D \cdot\left(\begin{array}{l}
\mathrm{x}^{\mathrm{x}} \\
\mathrm{y}^{\mathrm{x}}
\end{array}\right) \text { where } \mathrm{D}=\left(\begin{array}{ll}
1 & 0 \\
0 & r
\end{array}\right) \mathrm{r}=\text { anisotropy angle. }
$$

The ratio $r=R 1 / R 2$ then defined the scale factor which was used to stretch the axis. Thus, all points belonging to an ellipse was defined by the anisotropy axis R1 and R2 which was then transformd to a circle with radius R1. Finally, the geometric anistropy was descibed using

$\gamma(\mathrm{h})=\gamma(\|\mathrm{x}\|)=\sqrt{\mathrm{x}^{\mathrm{xx} 2}+\mathrm{y}^{\mathrm{xx} 2}}=\mathrm{y} \sqrt{\mathrm{x}^{\mathrm{T}} \mathrm{Q}^{\mathrm{T}} \mathrm{D}^{\mathrm{T}} \mathrm{DQx}}$ where $(\mathrm{h})=$ variogram value $\|\mathrm{x}\|=$ length of separation vector $x=\left(\begin{array}{l}x \\ y\end{array}\right) \quad \mathrm{x}^{\mathrm{T}}=(\mathrm{x}, \mathrm{y})$.

Thereafter the variogram to expressed the spatial variation and minimized the error in the forecasted canopy shaded $S$. damsnoum s.l. larval habitat values. Additionally, a by-product of our Ordinary kriging was a robust geographic mean function of the variogram which estimated the average of the predicted data points while filtering out spatial redundancy in the estimators. Pseudo-replicated errors in uncertainty estimation can substantially alter numerical predictions in risk-based residual forecasts derived from an autoregressive black flyrelated endmember models by inflating the value of test statistic. This increases the chance of a Type I error or an incorrect rejection of the null hypothesis; H0: no spatial autocorrelation [1]. Spatial autocorrelation is the correlation among values of a single variable strictly attributable to their relatively close locational positions on a two-dimensional surface, introducing a deviation from the independent observations assumption of classical statistics [2]. Our variogram also provided a weighted average, giving each predicted canopy shaded S. damnosum s.l. riverine habitat data point a weight proportional to its spatiotemporal field-sampled larval density estimate.

The estimated prediction variance was then calculated as: $s_{z}^{2}=\mathrm{w}_{1} S\left(d_{1 \mathrm{p}}\right)+\mathrm{w}_{2} S\left(d_{2 \mathrm{p}}\right)+\mathrm{w}_{3} S\left(d_{3 \mathrm{p}}\right)+\lambda$. When interpolating an object using the kriging method, an endmember estimated prediction variance estimate is always produced along with the interpolated data set $[1,47]$. As a result, an iso-surface plot of estimated prediction variance was rendered. The weights of the Ordinary krigedbased algorithm were then used to fulfill the unbiasedness condition in the spatial interpolation of the $S$. damnosum s.l. habitat endmember signature using $\sum \lambda=$ which was provided by the equation system by:

$$
\left(\begin{array}{c}
\lambda_{1} \\
\vdots \\
\lambda_{n} \\
1
\end{array}\right)=\left(\begin{array}{cccc}
\gamma\left(x_{1}, x_{1}\right) & \cdots & \gamma\left(x_{1}, x_{n}\right) & 1 \\
\vdots & \ddots & & \vdots \\
\gamma\left(x_{n}, x_{1}\right) & \cdots & \gamma\left(x_{n}, x_{n}\right) & 1 \\
1 & \cdots & 1 & 0
\end{array}\right)^{-1}\left(\begin{array}{c}
\gamma\left(x_{1}, x^{*}\right) \\
\vdots \\
\gamma\left(x_{n}, x^{*}\right) \\
1
\end{array}\right)
$$

as in Jacob et al. (2011c). An exponential model was then fitted to the semivariogram using a range of $72.6 \mathrm{~m}$ along the river corridor with a nugget of 0.21 (variance), a lag size of $11.5 \mathrm{~m}$ with 12 lags and a partial sill of 0.24 (variance). In the spatial analyst extension of ArcGIS $10.0^{\circledR}$, we calculated the new residual estimated prediction variance, (i.e., interpolation variance) and output it along with the traditional kriging variance. The $95 \%$ error bound based on the new variance was also reported. The spatial correlation error covariance matrix was then quantitated employing

$$
\operatorname{var}\left(\hat{Z}\left(x^{*}\right)-Z\left(x^{*}\right)\right)=\left(\begin{array}{c}
\gamma\left(x_{1}, x^{*}\right) \\
\vdots \\
\gamma\left(x_{n}, x^{*}\right) \\
1
\end{array}\right)\left(\begin{array}{cccc}
\gamma\left(x_{1}, x_{1}\right) & \cdots & \gamma\left(x_{1}, x_{n}\right) & 1 \\
\vdots & \ddots & \vdots & \vdots \\
\gamma\left(x_{n}, x_{1}\right) & \cdots & \gamma\left(x_{n}, x_{n}\right) & 1 \\
1 & \cdots & 1 & 0
\end{array}\right)^{-1}\left(\begin{array}{c}
\gamma\left(x_{1}, x^{*}\right) \\
\vdots \\
\gamma\left(x_{n}, x^{*}\right) \\
1
\end{array}\right)
$$


In the model a new canopy shaded S. damnosum s.l. riverine larval habitat spectral value was predicted at any new geographical location by combining the Gaussian prior with a Gaussian likelihood function for each of the canopied spectrally observed endmember values. The resulting posterior distribution was also Gaussian, with a mean and covariance that was simply computed from the observed new canopy-shaded riverine larval habitat values, their variance and the matrix derived from the prior. The models provided a method for not constraining the canopy shaded habitat endmember reference signature. In turn, the model allowed distance and direction in the interpolation process to be analyzed, which minimized the variance of unexpected error. The kriged smoothed maps displayed the spatial patterns from the canopy shaded S. damnosum s.l. endmember spectral signature. Furthermore, the model output was evaluated by a validation dataset based on the visual inspection of the predicted habitats. Our model revealed a $100 \%$ accuracy in the field-verified estimates generated from the residual output of the spatial interpolationbased algorithm.

Thereafter, we extracted the first few moments of the spectral radiance using the radiative transfer equation. The $\mu$ in the canopy shaded larval habitat model yielded $J_{v}=-\int I_{v} d=a \quad H_{v}=\frac{1}{2} \int_{-1}^{1} \mu I_{v} d \mu=\frac{b}{3}$, and $K_{v}=\frac{1}{2} \int_{-1}^{1} \mu^{2} I_{v} d \mu=\frac{a}{3}$ The Eddington approximation for our canopy shaded $S$. damnosum s.l. riverine larval habitat-related model was equivalent to setting $K_{v}=1 / 3 J_{v}$. Note that the first two moments had simple physical meanings [17]. The radiative transfer was then isotropically provided by $\mu \frac{d I_{v}}{d z}=-\dot{a}_{v}\left(I_{v}+B_{v}\right)+\sigma_{v}\left(J_{v}+I_{v}\right)$. Integrating over all angles in the riverine larval habitat model rendered $\frac{d H_{v}}{d z}=\alpha_{v}\left(B_{v}+J_{v}\right)$. Premultiplying by $\mu$, then integrated all angles in the model and rendered $\frac{d K_{v}}{d z}=-\left(\alpha_{v}+\sigma_{v}\right) H_{v}$. In this research it

resulted in the radiative diffusion equation $\frac{d^{2} J_{v}}{d z^{2}}=3 \alpha_{v}\left(\alpha_{v}+\sigma_{v}\right)\left(J_{v}-B_{v}\right)$.

\section{Discussion}

Initially, in this research, we aggregated multiple remotely synthesized explanatory sub-pixel explanatory predictor covariate coefficients from digital surface-based canopy shaded $S$. damnosum s.l. habitat LULC models constructed using QuickBird visible and NIR data. Digital surface models are topographic maps that provide a geometrically correct reference frame over which other satellite data layers can be draped [1]. A digitized grid-based matrix was then overlaid onto the georeferenced riverine habitat in ArcGIS for managing the spatiotemporal-sampled parameter estimators for generating an efficient mathematical-based sample frame. For remote identification of georeferenced arthropod-related infectious disease immature habitats, the first step is often to construct a discrete tessellation of the region $[2,48]$.

Normalized Difference Vegetation Index (NDVI) parameters were generated from the QuickBird data. We used radiances or reflectances from a red channel around $0.66 \mu \mathrm{m}$ and a near-IR channel around $0.86 \mu \mathrm{m}$. The red channel is located in the strong chlorophyll absorption region, while the near-IR channel is located in the high reflectance plateau of vegetation canopies [16]. Sub-meter spatial resolution reflectance is affected by BRDF and, thus, VI and nadir-normalized reflectance for identifying vector arthropodrelated immature habitat suitability [10]. We then constructed a robust Digital Elevation model (DEM) for identifying terrain-related geomorphological-based spectral explanatory predictor covariate coefficient estimates associated to the seasonal-sampled $S$. damnosum s.l. riverine habitats using the QuickBird derived LULC data. Once the LULC related predictors were determined, we employed $\mathrm{ENVI}^{\circ}$ technology and a radiative transfer model to extract and unmix the canopy-dependent emissivities from within the QuickBird canopy, shaded, S. damnsoum s.l., riverine habitat spectrum.

The three-dimensional radiative transfer model quantitated the physical processes describing the interaction of radiation with the diverse canopy components at foliage and canopy levels. Consequently, our physically based approach of coupled leaf and canopy radiative transfer models (RTMs) provided an adequate way to assess the $S$. damnosum s.l.-related canopy shaded variables, such as vegetation water content and LAI. Radiative transfer models have already been successfully employed with homogeneous canopies to derive quantitative information on canopy structure and foliage biochemistry [1] The three-dimensional canopy radiative transfer models parameterized the heterogeneous canopy structure appropriately. We generated canopy shaded image endmember from the decomposed QuickBird data of the georeferenced canopy shaded S. damnosum s.l. riverine larval habitat sampled using convex geometrical techniques. High space resolution satellite images, such as QuickBird, open new possibilities in spatiotemporal arthropod-related larval habitat predictive cartographic applications such as employing eigenfunction spatial filter orthogonal models with a logistic specification and a binary geographically autoregressively weighted matrices for spatially targeting prolific habitat based on field sampled count data [2]. In order to avoid biased residual interpolated forecasts, the spectral signiture was constructed using extremely precise spectral derivatives generated from multiple linear unmixing algorithms.

We employed the SPA algorithm and a robust geometric-optical model to spectrally quantitate areal fractions of sunlit canopy, sunlit background and shadow-related spectral endmember covariate coefficient estimates at su b-resolution sc ales. We encompassed the effects of the remotely captured riverine topographical data using the BRDF and the hemispherical reflectance (i.e., surface albedo) of the Precambrian rock and also of the rippled water decomposed canopyrelated photosynthetic components associated to the georeferenced $S$. damnosum s.l. riverine habitat in the geometric-optical model. In this research, the unmixing algorithm extracted canopy shaded endmember sub-pixel S. damnosum s.l. larval habitat data feature attributes from the QuickBird imaged scene without reducing the spectral data dimensionality. Our methodology used the canopy-oriented spectral angle and the spatial adjacency of the decomposed QuickBird visible and NIR data to constrain the selection of candidate sub-pixel data representing the canopy-related endmembers. We designed the SPA based on the assumption that there were many canopy-related targets that had locations of spatial continuity in the QuickBird image. Although there were some unique within canopy endmember values (e.g., Precambrian rock, turbid water sub-pixel estimates), many of the decomposed QuickBird endmembers had similar spectral properties. As such, the probability that two adjacent canopy shaded S. damnosum s.l. riverine larval habitat pixels were both spurious was low. Experiments on empirical ecological spatiotemporal-sampled datasets demonstrated that SPA can effectively extract canopy-related riverine larval habitat endmembers, while requiring minimal user interactions [5]. 
There are a number of potential improvements to SPA for extracting sub-meter resolution canopy-based shaded S. damnosum s.l. riverine larval habitat endmembers that require further research namely: 1) a means for the automatic determination of the spectral angle threshold $(t$ $\_$) and spatial threshold ( $t_{-}$pixel), and 2 ) a means to constrain the total number of the canopy-oriented endmembers in the QuickBird scene. In our research we found that 2.5 degrees for $\left(t_{-} \theta\right)$ and $\left(t_{-}\right.$pixel) provided good canopy-oriented endmember estimates. However, the selection of $\left(t_{-}\right.$pixel $)$and $\left(t_{-} \theta\right)$ may be scene dependent because of the varying spatial and spectral complexity in different within canopy-oriented immature Similium data feature attributes. Currently, the choice of these two thresholds is still arbitrary and it would be valuable in future research efforts to develop a more robust way to define canopyoriented riverine habitats endmember reflectance values. Finally, we do not yet have a definite means of constraining the number of canopy shaded $S$. damnosum s.l. riverine larval habitat endmembers in a given search, employing the simplex volume change whereby the search would offer a qualitative data assessment. The concept of virtual dimensionality (VD) has been proposed and proven useful for this purpose in recent years [18]. The use of the VD concept within SPA may allow the study of the relationship between VD and simplex volume in the future which may prove to be invaluable when constraining the number of canopy shaded spectral endmembers in a sub-meter resolution S. damnosum s.l. riverine habitat image.

Additionally, in this research we employed geometric optical model residuals to determine sunlit canopy component reflectance measurement values together with field measures of spatial ecohydrological background features of a Precambrian rock and ripple water components and their shadow reflectance for generating robust risks of hyperendemic transmission zones. Our models employed Boolean statistics for generating the proportion of sunlit or shadowed canopy and background as functions of view and illumination angles. In our research, Boolean set theory determined the shadowing behavior that produced the 'hotspot' through enhanced single scattering from the imaged riverine larval habitat [30]. Remotely-sensed spatiotemporal vector arthropod-related larval habitat canopy reflectance models have to deal with the interactions of light occurring within and between individual plant canopies which involves the surface scattering effect which coincidentally is the opposition effect or the geometric effect also known as "hotspot" effect [31]. In our model our hotspot function took several forms sometimes fully empirical, other times driven by the shape, orientation, and/or spacing of the surface materials. Regardless, an empirical angular dependence of irradiance to solar zenith angle for the canopy as a whole was quantitated. When the radiance is correctly spectrally quantitated, the data function can take several forms which include piece wise-linear, negative exponential, trigonometric and geometric functions [40,41]. In our model, the reference signature of a canopy shaded $S$. damnosum s.l. larval habitat was treated as an empirical function reducing the attenuation of exiting radiation as a function of the phase angle between illumination and view directions while capturing mutual shadowing endmember spectral data feature attributes. These parameter estimators were then included in an empirical phase function for further spectral endmember quantitation of the canopy reflected fractional proportions within the decomposed S. damnsoum s.l. larval habitat sub-pixel data. In most treatments, the phase function of the leaf surface is separated from a phase function that describes the hotspot [8].

Interestingly, we noted that the decomposed sub-meter resolution endmember data spectrally derived using the various combinations of unmixing algorithms coupled with a 3-D canopy radiative transfer model did determine foliar concentrations of pigments, leaf thickness, leaf angle distribution and other leaf chemistry and canopy spectra, of the georefernced canopy shaded S. damnosum s.l. riverine larval habitat. Our mechanistic radiative transfer model along with the SPA and the geometric-optical model spectrally quantitated nonhomogenous canopy-related explanatory predictor covariate coefficient estimates which in this research were employed an array of georeferenced observational parameter estimators distributed in $3 \mathrm{D}$ space. Additionally, our three-dimensional (3D) radiative transfer model accurately quantitated $3 \mathrm{D}$ and $1 \mathrm{D}$ photosynthetic covariates using solar radiation absorption, top-of-the-atmosphere upwelling and surface downwelling solar radiation calculations. Spatial variations of these same radiation components (i.e., absorption, upwelling, and downwelling), together with path length distributions were analyzed for different wavelengths to describe the main physical mechanisms at work in both modeling techniques. Our results revealed that the simple radiative transfer model can quantitate various plane-parallel (1D) assumptions but can under estimate shaded canopy absorption when compared to $3 \mathrm{D}$ computations. This discrepancy may have been caused by a complex interaction of gaseous absorption cloud droplet absorption and the solar zenith angle of the S. damnosum s.l. riverine larval habitat.

We then used a geometric-optical model to help remotely capture an accurate S. damnosum s.l. habitat reference signature which in turn, was used as a dependent variable in an ordinary kriged-based algorithm. We were able to interpolate the sub-meter resolution endmember signature of the canopied $S$. damnosum s.l. riverine larval habitat. Semivariograms were generated in $\operatorname{ArcGIS}^{\circledR}$ Spatial Analyst which modeled the structure of spectral variability in the decomposed habitat mixel. The semiovariogram was useful for summarizing the spatial continuity of the decomposed data. Optimal linear predictors were generated by incorporating the model of the covariance of the random function using a weighted moving average interpolation and the QuickBird endmember reference signature. The model measured the local variation in the reference signature as a function of distance and direction. There was no need to estimate a first-order trend; instead, the mean was estimated from the canopy shaded $S$. damnosum s.l. riverine larval habitat reference signature. Local deviations from the global mean were accounted for by the covariance model residual output. In the model a new habitat value was predicted at any new spatial locations by combining the Gaussian prior with a Gaussian likelihood function for each of the observed values. The resulting posterior distribution was also Gaussian, with a mean and covariance that was simply computed from the forecasted canopy shaded riverine larval habitat values, their variance and the matrix derived from the prior. The models provided a method for not constraining the canopy shaded $S$. damnosum s.l. riverine larval habitat endmember reference signature. In turn, the model allowed distance and direction in the interpolation process to be analyzed, which minimized the variance of unexpected error.The kriged smoothed maps displayed the spatial patterns from the $S$. damnsoum s.l. endmember spectral signature. Furthermore, the model output was evaluated by a validation dataset based on the visual inspection of the predicted habitats in a neighbouring riverine ecosystem. Our model revealed a $100 \%$ accuracy in the field-verified estimates generated from the residual output of the spatial interpolation-based algorithm.

There are many important and interesting findings we noted based on the residuals from our spatiotemporal canopy shaded $S$. damnosum s.l. riverine larval habitat geopredictive endmember models. First and foremost, field management of all riverine larval 
habitats may be unnecessary for obtaining specified objectives of reductions in incidence and prevalence of onchocerciasis.Th.s is especially important because riverine larval habitats are not uniform in productivity. Kriged estimated values may be sufficed to determine habitat suitability for inclusion into the endmember analyses. Second, given enormous variability observed in $S$. damnosum s.l. productivity between habitats, effective larval interventions may be a chieved by remotely targeting control efforts toward productive habitats. Third, for constructing robust geopredictive endmember risk models for targeting hyperendemic transmission zones based on aggregation of prolific habitats, seasonal larval habitat productivity must be based on the sampled data and ArcGIS delineated georeferenced spatial data feature attributes (e.g., larval density and surface size of habitats). Attacking multiple points in the transmission cycle may act synergistically and improve upon current single-tool interventions based on interpolation of a riverine larval habitat target signature.

In the future, time series dependent canopy shaded S. damnosum s.l. riverine larval habitat data feature attributes models may be applied to continuous spatial ecohydrological networks associated to extensive shaded land covers in riverine ecosystems, such as crop canopies and homogeneous grasslands next to forested rural river ecosystems for investigating other seasonal data feature attributes [e.g., flooded vegetation land use land cover (LULC) explanatory covariates)]. Further, particle swarm intelligence searching techniques for decomposing $S$. damnosum s.l. riverine larval habitat geopredictive canopy shaded endmember models may be forward in literature. Theoretically, this decomposition algorithm can take full advantage of neighbourhood information, making the riverine larval habitat decomposition results even more robust. The algorithm could then use the K-means clustering algorithm and a method of dynamic selection of canopied riverine larval habitat endmembers subsets to find the appropriate dataset of endmembers for a given set of multispectral images. The proposed algorithm has been successfully applied to test image sets from various platforms such as LANDSAT 5 MSS and NOAA's AVHRR. Thus, experiments need to be conducted to compare the PSOEMS with the multi-start Kmeans and other spectral unmixing techniques on various QuickBird derived seasonal canopy shaded S. damnosum s.l. riverine images Thereafter, performance of the PSO-EMS may be applied to submeter resolution imagery to determine the algorithm's mapping potential in seasonal geopredictive risk mapping targeting hyperendemic transmission zones based on aggregations of prolific habitats based on field-sampled count data and seasonal tabulated prevalence rates.

In conclusion, the QuickBird digitized gridded image provided an indication of the geographic location of the sampled canopy shaded $S$. damnosum s.l. habitat and its associated land cover attributes, specifically the Precambrian rock and rippled water components. The display of the habitat data in a QuickBird digitized-grid based format clearly identified highly productive canopy shaded S. damnosum s.l. habitats. QuickBird derived DEM statistics revealed that the geomorphological-related explanatory predictor slope was an important indicator variable in the riverine breeding study site. The QuickBird NDVI maps provided effective quantitative information of the LULC at the study site as the photosynthetic capacity of the surrounding and within the canopy vegetation-related feature data attributes was evaluated at the appropriate spatial scale. We noted that the majority of the reflection in the QuickBird NDVI model output was based on the visible, and NIR spectrum which was dependent on the canopy cover and the surface of the floating leaves associated to the georeferenced canopy shaded S. damnsoum s.l. riverine habitats.
Thereafter, ENVI ${ }^{\circledR}$ technology categorized individual pixels of the georeferenced habitats into separate spectral classes while converting remotely-sensed raster layers to vector coverages which classified the layers as ESRI shapefiles. The SPA-based analyses then identified the spectrally-dependent habitat pixel components that encompassed illumination geometrical values reflected from the Precambrian rock and ripple water at the vertices of the simplex.The algorithm identified the fractional presence of each canopy endmember spectra in the decomposed QuickBird canopy shaded S. damnosum s.l. habitat pixel.

Further, topographic effects on bidirectional and hemispherical reflectance generated from the larval habitat surface were then calculated with a geometric-optical model. The model used a hemispherical integration of a distorted BRDF which also provided an albedo estimate of the spatiotemporal-sampled riverine larval habitat, the Precambrian rock and the ripple water spectral reflectance values. We were able to also encompass the effects of the shaded habitat topography using the BRDF and the surface albedo generated from the riverine habitat canopy cover. The final model output identified the fractional presence of each canopy endmember of the $S$. damnosum s.l. habitat and its associated Precambrian rock and rippled water QuickBird spectral emissivity attributes based on proportions of sunlit canopy, sunlit background and shadow fraction. Thereafter, a kriged smoothed map displayed the spatial patterns of all productive $S$. damnosum s.l. riverine larval habitats based on the canopied endmember reference signature generated from the inverted model. We defined a practical range and the distance at which $95 \%$ of the sill was reached for the asymptotic variogram model. The combination of ENVI technology, a radiative transfer model SPA algorithm and a geometric optical model can predict $S$. damnosum s.l. larval habitats using Ordinary Kriged-based interpolator based on spatiotemporal field-sampled count data and decomposed sub-meter resolution QuickBird visible and NIR data.

\section{Acknowledgements}

This research was funded by the National Institute of Health Grant (Unnasch Thomas) University of South Florida.

\section{References}

1. Benjamin G Jacob, Daniel A Griffith, Joseph Mwangangi, David A Gathings Charles C Mbogo, et al. (2011) A Cartographic Analysis Using Spatial Filter Logistic Model Specifications for Implementing Mosquito Control in Kenya Urban Geography 32: 263-377.

2. Jensen JR (2005) Introductory digital image processing a remote sensing perspective. 3rd ed, Jacob BG, et al.(ed.), Prentice Hall series in geographic information science, Upper Saddle River, NJ Prentice Hall, 526

3. Widlowski JL, pinty B, gobron N, verstraete MM, Diner DJ, et al. (2004) Canopy structure parameters derived from multi-angular remote sensing data for terrestrial carbon studies. Climatic Change. 67: 403-415.

4. Gong P, Zhang A (1999) Noise effect on linear spectral unmixing. Geographic Information Sciences 5: 52-57.

5. Plaza A, Martinez P, Perez R, Plaza J (2004) A quantitative and comparative analysis of endmember extraction algorithms from hyperspectral data. IEEE Transactions on Geoscience and Remote Sensing 42: 650-663.

6. Borel CC, Gerstl SAW (1994) Nonlinear Spectral Mixing Models for Vegetative and Soil Surfaces. Remote Sensing of Environment. 47: 403-416.

7. Atkinson PM, Cutler MEJ, Lewis H (1997) Mapping sub-pixel proportional land cover with AVHRR imagery. International Journal of Remote Sensing 16: 917935

8. Brown M, Gunn SR, Lewis HG (1999) Support vector machines for optimal classification and spectral unmixing. Ecological Modeling 120: 167-179.

9. Carpenter GA, Gopal S, Macomber S, Martens S, Woodstock CE (1999) A neural network method for mixture estimation for vegetation mapping. Remote Sensing of Environment 70: 138-152. 
Citation: Jacob B, Novak RJ, Toe L, Sanfo MS, Caliskan S, et al. (2013) Unbiasing a Stochastic Endmember Interpolator Using ENVI Object-Based Classifiers and Boolean Statistics for Forecasting Canopied Simulium damnosum s.I. Larval Habitats in Burkina Faso. J Geophys Remote Sensing 2: 109. doi:10.4172/2169-0049.1000109

Page 36 of 36

10. Guilfoyle KJ, Althouse ML, Chang $\mathrm{Cl}$ (2001) A quantitative and comparative analysis of linear and nonlinear spectral mixture models using radial basis function neural networks. IEEE Transactions on Geoscience and Remote Sensing 39: 2314-2318

11. Nascimento JMP, Bioucas-Dias JMB (2005a) Does independent component analysis play a role in unmixing hyperspectral data? IEEE Transactions on Geoscience and Remote Sensing 43: 175-187.

12. Wang $F$ (1990) Fuzzy supervised classification of remote sensing images IEEE Transactions on Geoscience and Remote Sensing 28: 194-201.

13. Asner GP, Heidebrecht KB (2005) Desertification alters regional ecosystemclimate interactions. Global Change Biology 11: 182-194

14. Garcia M, Ustin SL (2001) Detection of interannual vegetation responses to climate variability using AVIRIS data in a coastal savanna in California. IEEE Transactions on Geoscience and Remote Sensing 39: 1480-1490.

15. Huang CY, Asner GP, Barger NN, Neff JC, Floyd ML (2010) Regiona aboveground live carbon losses due to drought-induced tree dieback in pinonjuniper ecosystems. Remote Sensing of Environment 114: 1471-1479.

16. Gilabert MA, Garcia-Haro FJ, Melia J (2000) A mixture modeling approach to estimate vegetation parameters for heterogeneous canopies in remote sensing. Remote Sensing of Environment 72: 328-345.

17. Hestir EL, Khanna S, Andrew ME, Santos MJ, Viers JH, et al. (2008) Identification of invasive vegetation using hyperspectral remote sensing in the California Delta ecosystem. Remote Sensing of Environment, 112: 4034-4047.

18. Painter TH, Dozier J, Roberts DA, Davis RE, Green RO (2003) Retrieval of subpixel snow-covered area and grain size from imaging spectrometer data. Remote Sensing of Environment 85: 64-77.

19. Li L, Ustin SL, Lay M (2005) Application of multiple endmember spectral mixture analysis (MESMA) to AVIRIS imagery for coastal salt marsh mapping: a case study in China Camp, CA, USA. International Journal of Remote Sensing 26: 5193-5207.

20. Liu JG, Miller JR, Haboudane D, Pattey E, Hochhaim K (2008) Crop fraction estimation from casi hyperspectral data using linear spectral unmixing and vegetation indices. Canadian Journal of Remote Sensing 34: S124-S138.

21. Peddle DR, Smith M (2005) Spectral mixture analysis of agricultural crops: endmember validation and biophysical estimation in potato plots. International Journal of Remote Sensing 26: 4959-4979.

22. Eckmann TC, Still CJ, Roberts DA, Michaelsen JC (2010) Variations in subpixe fire properties with season and land cover in Southern Africa. Earth Interactions 14: $1-29$

23. Jia GJ, Burke IC, GoetzAFH, Kaufmann MR, Kindel BC (2006) Assessing spatial patterns of forest fuel using AVIRIS data. Remote Sensing of Environment 102: 318-327.

24. Katra I, Lancaster N (2008) Surface-sediment dynamics in a dust source from spaceborne multispectral thermal infrared data. Remote Sensing of Environment 112: 3212-3221.

25. Roberts DA, Gardner M, Church R, Ustin S, Scheer G, et al. (1998) Mapping Chaparral in the Santa Monica Mountains Using Multiple Endmember Spectral Mixture Models. Remote Sensing of Environment, 65: 267-279.

26. Asner GP, Keller M, Pereira R, Zweede JC, Silva JNM (2004) Canopy damage and recovery after selective logging in Amazonia: Field and satellite studies. Ecological Applications 14: S280-S298.

27. Peddle DR, Hall FG, LeDrew EF (1999) Spectral mixture analysis and geometricoptical reflectance modeling of boreal forest biophysical structure. Remote Sensing of Environment 67: 288-297.

28. Somers B, Delalieux S, Verstraeten WW, van Aardt JAN, Albrigo G, et al. (2010a) An automated waveband selection technique for optimized hyperspectral mixture analysis. International Journal of Remote Sensing 31: 5549-5568.

29. Mertes LAK, Smith MO, Adams JB (1993) Estimating suspended sediment concentrations in surface waters of the Amazon river wetlands from Landsat images. Remote Sensing of Environment 43: 281-301.

30. Svab E, Tyler AN, Preston T, Presing M, Balogh KV (2005) Characterizing the spectral reflectance of algae in lake waters with high suspended sediment concentrations. International Journal of Remote Sensing 26: 919-928.

31. Mertes LAK (2002) Remote Sensing of riverine landscapes. Freshwater Biology 47: 799-816.

32. Bedini E (2009) Mapping lithology of the Sarfartoq carbonatite complex, southern West Greenland, using HyMap imaging spectrometer data. Remote Sensing of Environment 113: 1208-1219.

33. Green RO, Eastwood ML, Sarture CM, Chrien TG, Aronsson M, et al. (1998) Imaging spectroscopy and the Airborne Visible Infrared Imaging Spectrometer (AVIRIS). Remote Sensing of Environment 65: 227-248.

34. Phinn S, Stanford M, Scarth P, Murray AT, Shyy PT (2002) Monitoring the composition of urban environments based on the vegetation-impervious surface soil (VIS) model by subpixel analysis techniques. International Journa of Remote Sensing 23: 4131-4153.

35. Rashed T, Weeks JR, Roberts DA, Rogan J, Powell R (2003) Measuring the physical composition of urban morphology using multiple endmember spectral mixture models. Photogrammetric Engineering and Remote Sensing 69: 1011 1020.

36. Weng QH, Lu DS, Schubring J (2004) Estimation of land surface temperature vegetation abundance relationship for urban heat island studies. Remote Sensing of Environment 89: 467-483.

37. Wu CS, Murray AT (2003) Estimating impervious surface distribution by spectral mixture analysis. Remote Sensing of Environment 84: 493-505.

38. Fischer EM, Pieters CM (1994) Remote determination of exposure degree and iron concentration of lunar soils using VIS-NIR spectroscopic methods. Icarus 111: 475-488.

39. Li L, Mustard JF (2003) Highland contamination in lunar mare soils: Improved mapping with multiple endmember spectral mixture analysis (MESMA). Journal of Geophysical Research Planets, pp. 108.

40. Pinet PC (1995) Spectroscopic imaging of solid planetary surfaces. Tridimensional optical spectroscopic methods in Astrophysics 71: 294-297.

41. Small C (2001) Estimation of urban vegetation abundance by spectral mixture analysis. Int J Remote Sens 22: 1305-1334.

42. Weng Q, Lu D, Schubring J (2004) Estimation of land surface temperaturevegetation abundance relationship for urban heat island studies. Remote Sens Environ 89: 467-483.

43. Geman S, Geman D (1984) "Stochastic Relaxation, Gibbs Distributions and the Bayesian Restoration of Images," IEEE Trans Pattern Anal Mach I ztel 6: 721-741.

44. Roberts DA, Quattrochi DA, Hulley GC, Hook SJ, Green RO (2012) Synergies between VSWIR and TIR data for the urban environment: an evaluation of the potential for the Hyperspectral Infared Imager (HyspIRI) decadal survey mission. Remote Sens Environ 117: 83-101.

45. Kettig RL, Landgrebe DA (1976) Computer Classification of Remotely Sensed Multispectral Image Data by Extraction and Classification of Homogeneous Objects. IEEE Transactions on Geoscience Electronics 1: 19-26.

46. Jacob BG, Novak RJ, Toe L, Sanfo MS, Afriyie AN, et al. (2012) QuasiLikelihood Techniques in a Logistic Regression Equation for Identifying Simulium damnosum s.I. Larval Habitats Intra-cluster Covariates in Togo. Geo Spat Inf Sci 15: 117-133

47. Somers B, Delalieux S, Verstraeten W, Coppin P (2009) A conceptua framework for the simultaneous extraction of sub-pixel spatial extent and spectral characteristics of crops. Photogrammetric Engineering and Remote Sensing 75: 57-68.

48. Lobell DB, Asner GP (2002) Moisture effects on soil reflectance. Soil Science Society of America Journal 66: 722-727.

49. Nichols J, (2012) Remote sensing of river stage using the cross sectiona inundation area - river stage relationship (IARSR) constructed from digital elevation model data. Hydrol Process.

50. Jupp D, Walker J, Penridge L (1986) Interpretation of vegetation structure in Landsat MSS imagery: A case study in disturbed semi-arid eucalypt woodlands Part 2. Model-based analysis. Journal of environmental management 23: 35-57.

51. Li Strahler AH, Jupp DLB (1990) Modeling bidirectional reflectance of forests and woodlands using Boolean models and geometric optics. Remote Sensing of Environment 34: 153-166.

52. Strahler AH, Jupp DL (1990) Modeling bidirectional reflectance of forests and woodlands using Boolean modelsand geometric optics. Remote Sens Environ 34:153-166 